Graydon L. Yoder Jr. Warren M. Rohsenow

Report No. 85694-103

Contract No. NSF Grant 76-82564-CME

Heat Transfer Laboratory

Department of Mechanical Engineering Massachusetts Institute of Technology Cambridge, Massachusetts 02139

March 1980

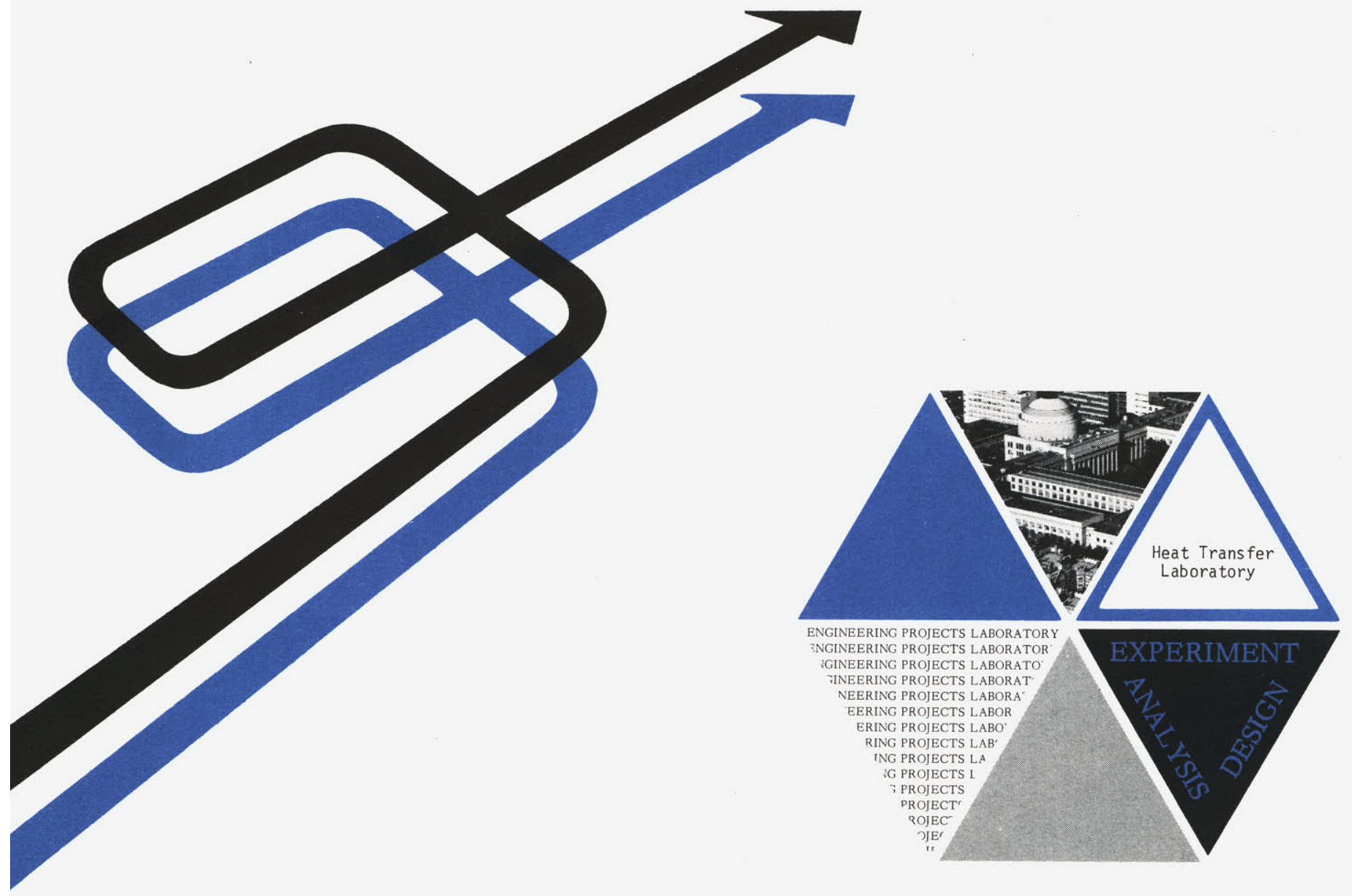


TECHNICAL REPORT NO. 85694-103

\title{
DISPERSED FLOW FILM BOILING
}

\author{
by
}

$$
\begin{gathered}
\text { Graydon L. Yoder Jr. } \\
\text { Warren M. Rohsenow }
\end{gathered}
$$

\author{
Sponsored by \\ National Science Foundation \\ Contract No. NSF Grant ENG 76-82564 \\ D.S.R. Project No. 85694 \\ March 1980 \\ Department of Mechanical Engineering \\ Massachusetts Institute of Technology \\ Cambridge, Massachusetts 02139
}


ABSTRACT

Dispersed flow consists of small liquid droplets entrained in a flowing vapor. This flow regime can occur in cryogenic equipment, in steam generators, and during nuclear reactor loss of coolant accidents. A theoretical analysis of dispersed flow film boiling has been performed using mass, momentum and energy conservation equations for both phases.

A numerical solution scheme, including wall-to-drop, vapor to drop, and wall-to-vapor heat transfer mechanisms was used to predict wall temperatures for constant heat flux, vertical upflow conditions. Wall temperature predictions were compared to liquid nitrogen, Freon-12 and water data of four separate investigators with reasonable results.

A local conditions solution was developed by simplifying the governing equations, using conclusions from the numerical model. A non-dimensional group was found which solely determined the non-equilibrium with the flow, and allowed hand calculation of wall temperatures. The local conditions solution was compared to data taken by five investigators with good results. 


\section{ACKNOWLEDGEMENTS}

The authors are grateful to Professors Peter Griffith, Bora Mikic, and Anin Sonin for their help and advice. Thanks also to Professor Gail McCarthy and Mr. Larry Hull for their comments and suggestions. The typingwas done by Ms. Gisela Rinner whose assistance is sincerely appreciated. 
This research was supported by a grant from the National Science Foundation. 
TABLE OF CONTENTS

$\underline{\text { Page }}$

Abstract 2

Acknow ledgement

List of Figures $\quad 8$

List of Tables $\quad 12$

Nomenclature 13

$\begin{array}{lll}\text { CHAPTER I: } & \text { INTRODUCTION } & 18\end{array}$

1.1 Dispersed Flow Heat Transfer 18

1.2 Review of Related Work 24

1.3 Objectives of Research 30

$\begin{array}{ll}\text { CHAPTER II: } & \text { FORMATION OF DISPERSED FLOW, } \\ & \text { DROP SIZING MECHANISMS }\end{array}$

2.1 Formation and Breakup of Liquid Droplets 31

2.1a Inverted Annular Flow 38

2.1b Annular Flow 41

2.2 Average Drop Size at Burnout 48

2.2a Inverted Annular Flow 48

2.2b Annular Flow 48 
Page

CHAPTER III: DISPERSED FLOW HEAT TRANSFER MODEL

3.1 Formulation 52

3.1a Dryout Conditions 59

3.1b Governing Equations 61

3.1c Correlations 69

3.2 Numerical Solution and Results 89

3.2a Comparison of Numerical Model with Data 91

3.2b Contribution of Individual Heat Transfer Mechanisms

104

3.3 Discussion of the Numerical Solution 112

CHAPTER IV: LOCAL CONDITIONS SOLUTION 116

4.1 Formulation $\quad 116$

$\begin{array}{ll}4.1 \text { a Dryout Conditions } & 117\end{array}$

4.1b Governing Equations $\quad 127$

4.2 Calculation Procedure 147

4.2a Calculation of Initial Drop Size
at $X=X_{b}$

4.2b Calculation of Constant $K \quad 153$

4.2c Calculation of Local Wall Temperature 155

4.3 Local Conditions Solution and Results 156

4.3a Comparison of the Local Conditions
Model with Data

4.3b Effect of a Variation in the Constant $K 169$ 
$\underline{\text { Page }}$

4.4 Discussion of the Local Conditions Solution

APPENDICES

Appendix Al Free Stream S1ip Determination 178

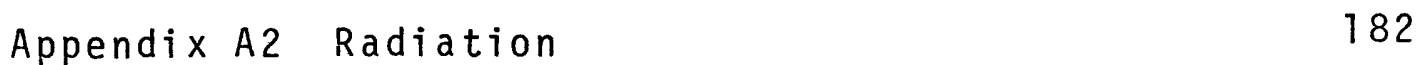

$\begin{array}{lll}\text { Appendix A3 Effect of Drops on Boundary } & 184 \\ \text { Layer Growth } & \end{array}$

Appendix A4 Sample Calculation 193

Appendix A5 Droplet Entrainment and Deposition 200 
LIST OF FIGURES

Figure

Page

$\begin{array}{lll}\text { 1-1 Annular Flow } 20 & \end{array}$

1-2 Inverted Annular Flow 21

2-la Helmholtz-Instability-Drop Formation 32

2-1b Film Slip Weber Number-Drop Formation 33

2-1c Weber Number Breakup 34

2-2 Void Fraction Comparison 37

2-3 Drop Formation in Inverted Annular Flow 39

2-4 Drop Sizing Mechanisms 43

2-5 Drop Sizing Mechanisms: Fr-12 46

2-6 Drop Sizing Sequences 47

3-1 Heat Transfer Mechanisms in Dispersed Flow 53

3-2 Drop Impaction Temperature Profile Droplet Dominated

3-3 Drop Impaction Temperature Profile Convection Dominated

3-4 Axial Conduction in the Tube 88

3-5 Comparison of Numerical Model with

Forslund's Nitrogen Data

3-6 Comparison of Numerical Model with

Forslund's Nitrogen Data 93

3-7 Comparison of Numerical Model with Groeneveld's Freon-12 Data

3-8 Comparison of Numerical Model with Bennett's Water Data 
Figure

Page

3-9 Comparison of Numerical Model with Bennett's Water Data

3-10 Effect of Altering Vapor Heat Transfer Coefficients: Water

3-11 Comparison of Numerical Model with Cumo's Freon-12 Data

3-12 Comparison of Numerical Model with Cumo's Freon-12 Data

3-13 Comparison of Complete Equilibrium Prediction to Cumo's Freon-12 Data

3-14 Effect of Axial Conduction in the Tube Wall

3-15 Actual Heat Flux Entering Fluid with Conduction Present in the Tube Wall

3-16 Ratio of Wall-to-Drop Heat Transfer to Total Drop Heat Transfer: Equation $(3-17)$

3-17 Ratio of Drop-Wall Heat Transfer to Total Heat Transfer: Equation (3-28)

4-1 Drop Diameter History After Burnout

4-3 ( $\left.x_{e q}-x_{b}\right)$ vs. $x$ for $x_{b}=0.1$ 
Figure

Page

$4-9$

$\left(x_{e q}-x_{b}\right)$ vs. $x$ for $x_{b}=0.7$

144

$4-10$

$\left(x_{e q}-x_{b}\right)$ vs. $x$ for $x_{b}=0.8$

145

4-11

$\left(x_{e q}-x_{b}\right)$ vs. $x$ for $x_{b}=0.9$

146

4-12 Comparison of Local Conditions Model with Forsiund's Nitrogen Data

4-13 Comparison of Local Conditions Model with Forslund's Nitrogen Data

158

4-14 Comparison of Local Conditions Model with Bennett's Water Data

4-15 Comparison of Local Conditions Model with Bennett's Water Data

4-16 Comparison of Local Conditions Model with Groeneveld's Freon-12 Data

4-17 Comparison of Local Conditions Model with Cumo's Freon-12 Data

4-18 Comparison of Local Conditions Model with Cumo's Freon-12 Data

4-19 Comparison of Local Conditions Model with Ueda's Freon-113 Data

4-20 Comparison of Local Conditions Model with Ueda's Freon-113 Data

4-21 Ueda's Apparatus

4-22 Effect of a Variation in the Constant $K$

A1-1 Comparison of Numerically Calculated Slip and Slip Calculated from Equation (3-5): Equilibrium

Al-2 Comparison of Numerically Calculated Slip and Slip Calculated from Equation (4-6): Non-Equilibrium 
A3-1 Boundary Layer Growth with a Sink

Present

A3-2 Comparison of Integral Boundary Layer Solution with Yao's Numerical Calculations

A5-1 Drop Deposition in Annular Flow 201

A5-2 Hewitt's Entrainment Curve 203

A5-3 Single Sized Droplet Group History 204

A5-4 Cumulative Mass Distribution Function 206 


\section{LIST OF TABLES}

$\underline{T a b} 7 \mathrm{e}$

Page

3-1 Single Phase Vapor Nusselt Number Correlations

4-1 Comparison of Methods for Calculating

154

A2-1 Radiative Heat Exchange 183

A4-1 Calculated Wall Temperatures 199 


\section{NOMENCLATURE}

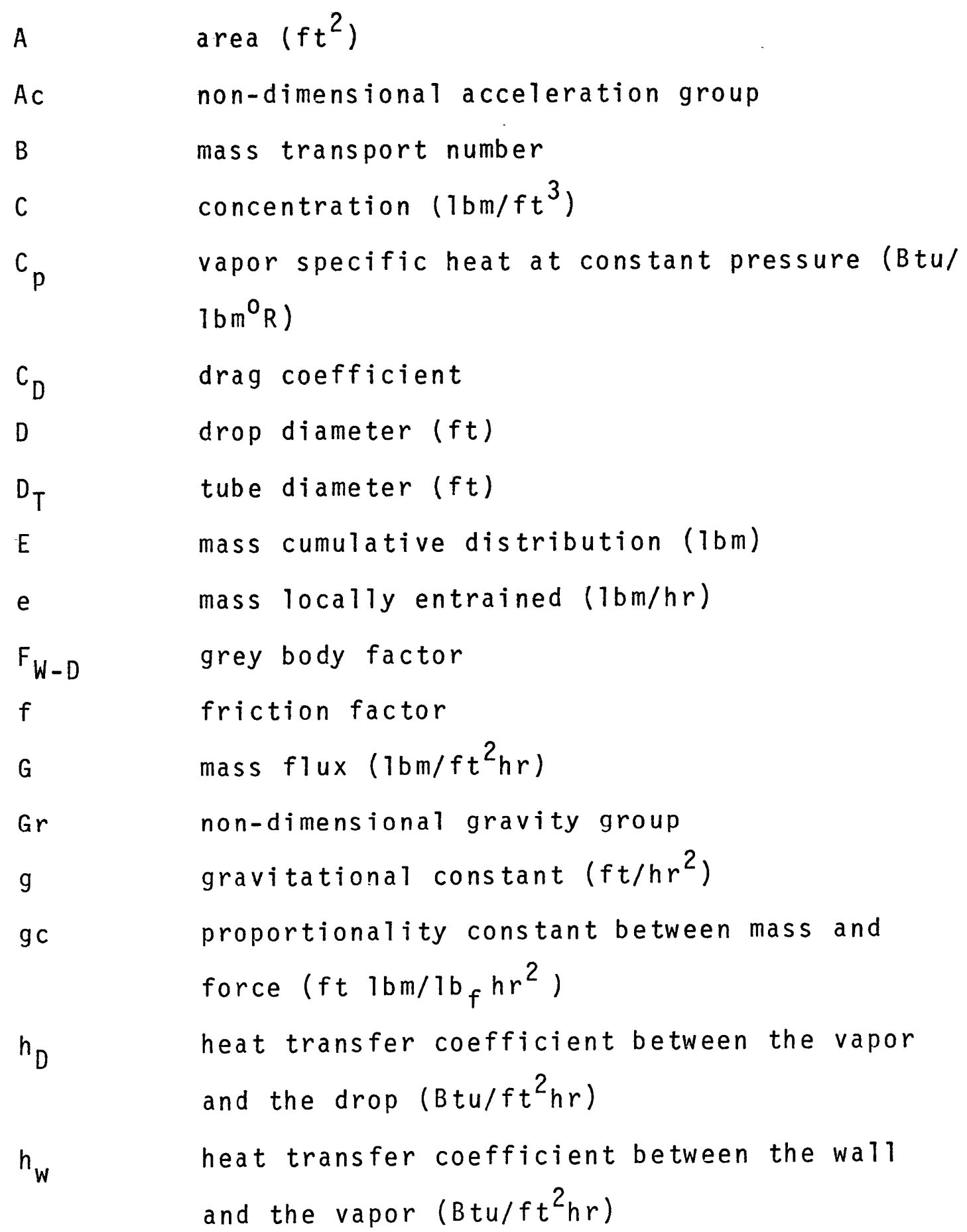




\begin{tabular}{|c|c|}
\hline I & integral constant \\
\hline K & non-equilibrium constant \\
\hline k & thermal conductivity (Btu/fthr$\left.{ }^{0} \mathrm{R}\right)$ \\
\hline M & droplet loading parameter \\
\hline m & mass $(1 \mathrm{bm})$ \\
\hline$\dot{\mathrm{m}}$ & mass flow rate $(1 \mathrm{bm} / \mathrm{hr})$ \\
\hline$n$ & number density $\left(\# / f t^{3}\right)$ \\
\hline$\dot{n}$ & number flow rate $(\# / h r)$ \\
\hline $\mathrm{Nu}$ & Nusselt number \\
\hline $\mathrm{Nu}$ & zero mass transfer Nusselt number \\
\hline $\operatorname{Pr}$ & Prandt 1 number \\
\hline Q & heat flow rate (Btu/hr) \\
\hline$Q_{C}$ & conduction heat flow rate $(B t u / h r)$ \\
\hline$Q_{r}$ & elemential heat flow rate $(B t u / h r)$ \\
\hline$q$ & heat flow rate $(B t u / h r)$ \\
\hline $\mathrm{R}$ & drop extension radius $(f t)$ \\
\hline Re & Reynolds number \\
\hline $\operatorname{Re}_{D}^{\prime}$ & Reynolds number defined by Equation (4-22) \\
\hline S & slip ratio \\
\hline $\mathrm{T}$ & temperature $\left({ }^{O} \mathrm{R}\right)$ \\
\hline$t$ & time $(h r)$ \\
\hline$t$ & tube thickness $(f t)$ \\
\hline V & velocity (ft/hr) \\
\hline
\end{tabular}


$-15-$

$\begin{array}{ll}v^{+} & \text {non-dimensional velocity } \\ \text { We } & \text { Weber number } \\ y & \text { thermodynamic quality } \\ z & \text { distance from the wall (ft) } \\ z_{b} & \text { axial coordinate }(f t) \\ z^{+} & \text {distance to burnout from beginning of }\end{array}$

Greek

$\alpha$

B

$\Gamma$

$\gamma$

$\delta$

$\varepsilon$

K

$\mu$

u

$\xi$

$\xi^{+}$

$\pi$

$\rho$

$\sigma$ void fraction

coefficient

droplet deposition ( $1 \mathrm{bm} / \mathrm{hr})$

emissivity

drop wall separation ( $f t)$

effectiveness

mass transfer coefficient ( $f t / h r)$

viscosity $(1 \mathrm{bm} / \mathrm{fthr})$

local velocity (ft/hr)

boundary layer thickens ( $f t$ )

non-dimensional boundary layer thickness

$2[\operatorname{Arcsine}(1)]$

mass density $\left(1 \mathrm{bm} / \mathrm{ft}^{3}\right)$

surface tension $\left(1 b_{f} / f t\right)$ 
$\tau$

$\tau_{0}$

$\tau^{+}$

$\psi$

Subscripts

a

B

b

c

D

eq

$f$

$\mathrm{hn}$

I

$$
\ell
$$

$\mathrm{N}$

0

R

S

TA

T

$t$

v

VD non-dimensional surface tension group

shear stress at the wall $\left(1 b_{f} / f t^{2}\right)$

non-dimensional relaxation time

Stefan-Boltzman constant (Btu/hr $\mathrm{ft}^{2}{ }{ }^{{ }_{R}}{ }^{4}$ )

intercept: pre-burnout

bulk

burnout

critical

drop

equilibrium

film

homogeneous nucleation

intercept: post burnout

liquid

node number

average at burnout

radiation

saturation

tangent point

total

tube

vapor

vapor-to-drop 


$\begin{array}{ll}\text { W } & \text { wall } \\ \text { WV } & \text { wall-to-vapor } \\ \text { WD } & \text { wall-to-drop }\end{array}$

\section{Superscripts}

III per unit volume

II per unit area per unit length 


\section{CHAPTER I}

\section{INTRODUCTION}

\subsection{Dispersed Flow Heat Transfer}

Two phase heat transfer has many applications for both heating and cooling. Cryogenic machinery, steam generators, wet steam turbines, and boiling water nuclear reactors all incorporate two phase heat transfer to some extent. In recent years, reactor safety analysis has spurred even more research in the area.

Because a phase change is occuring, high heat transfer rates are possible with low temperature differences. However, the heat transfer characteristics are highly dependent on the type of two phase flow regime present. Regimes are normally characterized by the distribution of liquid and vapor in the flow.

Dispersed flow is a regime which consists of small liquid droplets entrained by flowing vapor. The high material temperatures which characterize this flow pattern make it significant in any two phase flow heat transfer analysis. Wall temperatures are higher than the Leidenfrost temperature, so drops do not wet the wall. However, the presence of liquid can alter the vapor heat transfer by acting as a sink within the flow.

Dispersed flow film boiling is normally found in combination with other two phase boiling regimes. Two 
general types of flow patterns have been observed to precede the formation of dispersed flow [1]. The presence of one or the other of these regimes depends on the heat flux, and/or the wall temperature preceding flow initiation.

Low initial wall temperatures or low heat fluxes lead to the flow regime shown in Figure 1-1. As liquid entering the bottom of the tube is heated, vapor bubbles begin to form at the walls. In the nucleate boiling region, liquid remains in contact with the walls assuring good heat transfer and low wall temperatures. As more vapor is generated vapor collects in the center of the tube, surrounded by a liquid film attached to the wall. This is termed annular flow. Because of the large density difference between the vapor and liquid, the vapor travels at a much higher velocity than the liquid. Instabilities on the liquid surface cause droplets to be torn from the film and entrained in the vapor core. Eventually, evaporation and entrainment deplete the liquid on the wall and dryout or burnout occurs.

Inverted annular flow, the second flow pattern which may precede dispersed flow, occurs when wall temperatures are high previous to flow initiation, or when high heat fluxes are imposed. This pattern is shown in Figure 1-2. In inverted annular flow, the burnout point is very near 


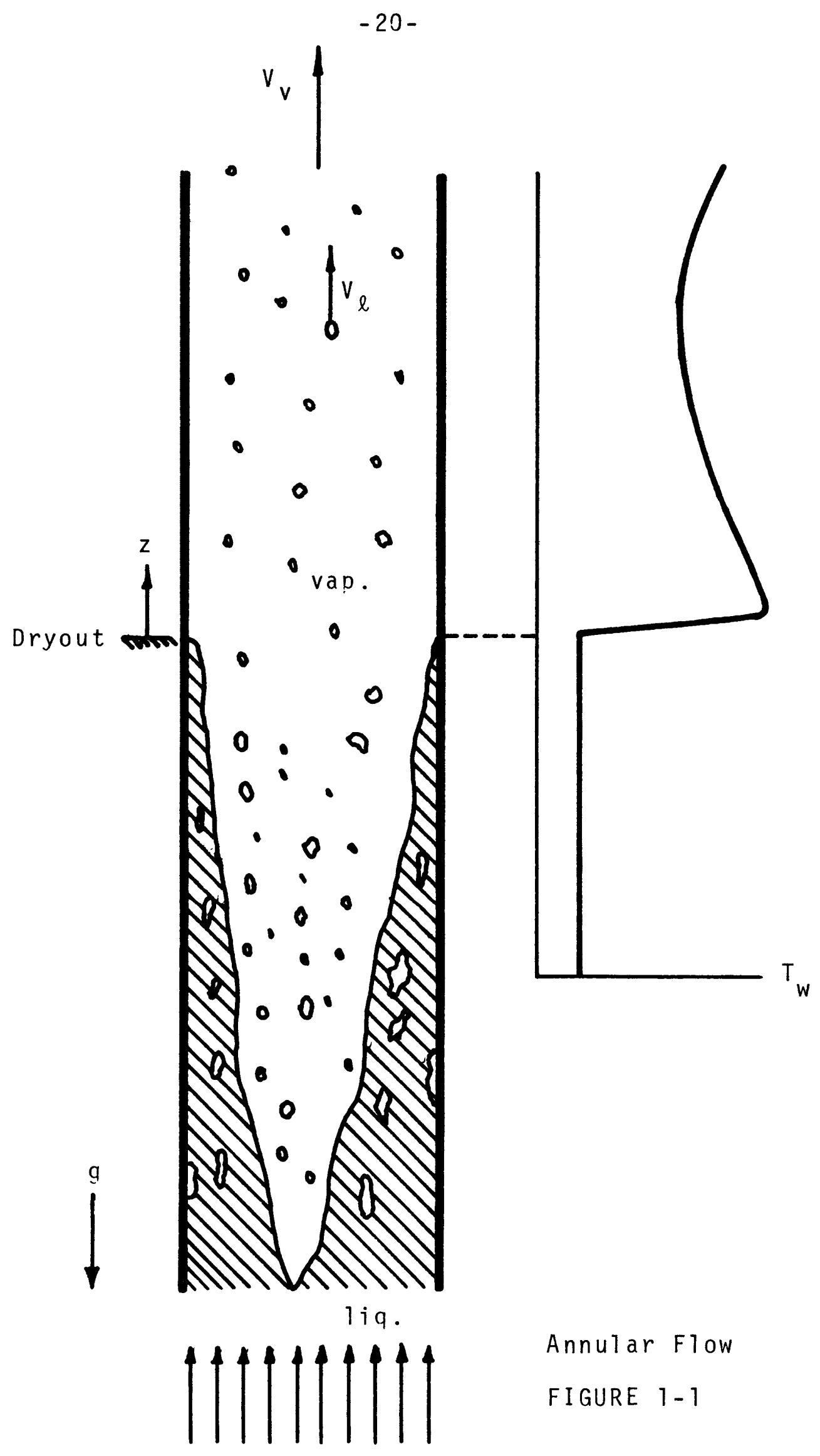




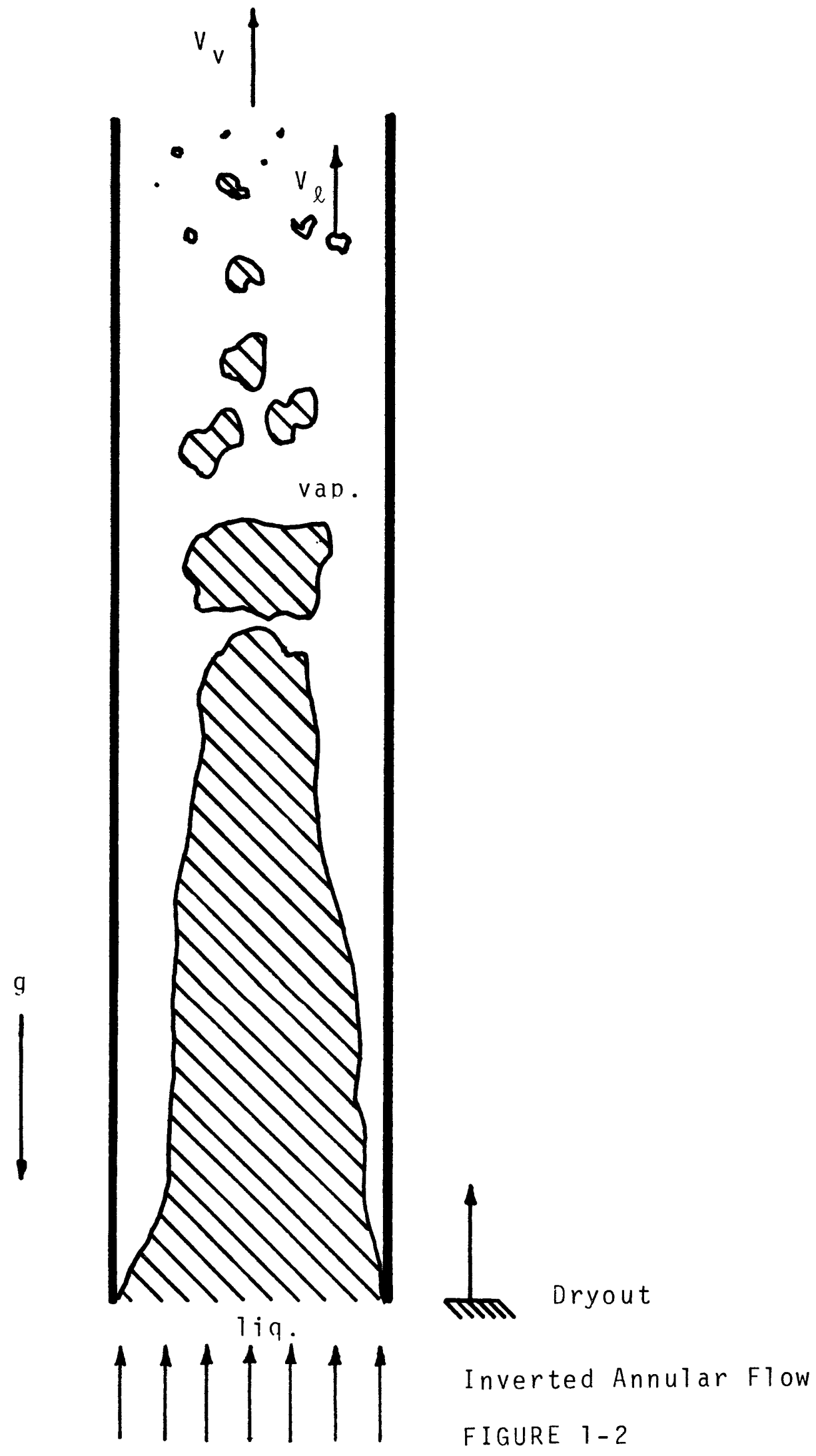


the beginning of the heated section. Wall temperatures are high enough to cause the liquid to form a core in the center of the tube, with a vapor annulus next to the wall. Vapor velocities are again much higher than the liquid velocities, and eventually the liquid core becomes unstable. Once the core ruptures, droplets rapidly form and dispersed flow is established.

The qualitative wall temperature profile in Figure 1-1 shows low wall temperatures while liquid remains in contact with the wall. Once the dryout point is reached, the primary heat transfer is between the wall and vapor. Observed temperatures in this region are high, sometimes high enough to cause material failure. Thus, dispersed flow heat tranfer must be correctly understood in order to safely design any equipment operating in this region.

The presence of 1 iquid in the flow poses the primary difficulty in analyzing any two phase system. The interaction between the two phases becomes important if any accuracy is desired when modeling flows of this type. In dispersed flow, the vapor flowing volume is much higher than the liquid volume (void fractions are typically $90 \%$ to $100 \%$ ), however, the liquid mass flow can be comparable to the vapor mass flow due to the large density ratio. Separation of the two phases must be carried out for both 
energy and momentum analysis. The liquid can travel at a velocity differing from the vapor velocity, and heat transferred from the walls must first heat the vapor before evaporating the drops. Vapor temperatures are normally higher than the liquid temperatures, and non-equilibrium exists in the flow. In addition, complications arise if drop-wall interactions are included in the analysis. 


\subsection{Review of Related Work}

\section{EXPERIMENTAL}

Dispersed flow experimental data is available for many fluids over a large range of flow conditions and differing flow geometries. Bennett et al [1] has taken wall temperature data under constant heat flux conditions using water as the fluid. Forslund [3] and Hynek [1] have both taken wall temperature data for nitrogen. Groene:'eld [5] and Cumo et al [6] have published similar data for Freon 12 while Koizumi et al [7] used Freon 113, and determined constant heat flux wall temperatures.

Basically two types of approaches have been utilized in analyzing dispersed flow heat transfer data. They can conveniently be divided into correlative and phenomenological analyses.

\section{CORRELATIVE}

Correlations are normally developed using data from a limited number of sources, and as such are typically limited to a range of flow conditions and one fluid. However, they are convenient to use because they do not require a computer solution and are therefore very attractive.

Many such correlations begin with an accepted equation for pure vapor such as the McAdams or Dittus-Boelter correlation and modify it to account for such things as 
non-equilibrium, slip (the ratio of vapor to liquid velocities), and entrance length effects in the flow.

Miropolski [8] developed a film boiling correlation for water including a correction for slip in the flow. He modified the Dittus-Boelter correlation to describe the heat transfer to pure steam, and applied the resulting correlation to two phase steam-water flows.

Polomik et al [9] modified the Colburn equation for $100 \%$ steam to correlate high pressure steam-water data. Bevi et al [10] also used a modified Colburn equation to predict two phase heat transfer data.

Groeneveld[11] presented a heat transfer correlation including a slip factor similar to Miropolki's. The equation was generalized for different flow geometries by determining a set of constants appropriate for each geometry.

Mattson et al [12] used a regression analysis on several data sets for different flow geometries and developed a separate correlation for each geometry. His correlations included a heat flux effect and a gas conductivity to critical point conductivity ratio.

More recently, Chiang et al [13] developed a correlation including both vapor and droplet-wall effects and compared it to water data taken using liquid sodium as the 
heat source. Saha [14] has developed a correlation based on vapor generation rates in steam-water flow.

A partial list of available correlations is presented by Groeneve $1 \mathrm{~d}[15]$.

\section{PHENOMENOLOG I CAL}

Most phenomenological approaches begin with an assumed heat transfer model, and follow the flow as it moves down the tube. This requires a step by step solution scheme and must be implemented on a computer. The advantage in this approach is that it accounts for specific heat transfer mechanisms within the flow.

Early work using this technique was performed by Dugall [16] who modeled the heat transfer as though it were to pure vapor flowing at the local vapor velocity. Laverty [17] extended this analysis to include a mechanism for droplet-vapor heat exchange. Forslund [3] and Bennett [2] both developed a step by step solution scheme which accounted for slip between the drops and vapor, and allowed for droplet breakup as it traveled down the tube. Forslund also included a drop-wall interaction term which allowed heat to be transferred directly between the droplet and wall upon contact. Hynek [1] extended this analys is to include the effect of a twisted tape on the heat transfer. 
He also observed the existance of both annular and inverted annular flow patterns at burnout. Groeneveld[5] used a similar calculation technique, but modified the wall-todroplet heat transfer term. Plummer [18] developed a simplified approach to heat transfer calculations based on this type of numerical scheme. More recently, Koizumi et al [7] has used this approach to predict wall temperatures for Fr-113 two phase flow.

The accuracy of these models depend on how well individual heat transfer mechanisms are understood. Analysis has focused primarily on the droplets in the flow. One area of major concern has been the heat transfer between the heated wall and the drops upon impact. A convenient measure of the drop-wall heat transfer is the effectiveness, the ratio of the amount of heat transferred to the drop to that which would be required to completely evaporate the drop.

Experimental measurements of heat transfer to drops impacting a heated surface have been conducted by Pederson [19]. His results show a distinct drop in heat transfer effectiveness as the surface temperature is increased, as do practically all other experimental results. Ueda et al [20] has studied the heat transfer characteristics of water and Freon 113 droplets impinging on a hot surface. 
Experimental results for water droplets show the effectiveness ranging from . $5 \%$ to $2 \%$ for surface temperatures above $90^{\circ} \mathrm{C}$. Styriocivich et al [21] studied the same phenomenon, using water droplets. Their photographic results indicate drop contact with the heated surface even at high surface temperatures. Effectiveness was in the range of 10\%. McCarthy [22] also investigated impingement of water droplets. Her experimental results show heat transfer effectiveness on the order of $10 \%$ also, but she concluded that much of the heat transfer was due to vapor entrainment by the droplet stream. She developed a dynamic model which agreed well with her experimental results once the entrainment effect was deducted.

Particle deposition in turbulent flows has been studied by Liu and Ilori [23]. Their deposition model is based on the eddy diffusivity present in turbulent flows. Visual studies of drop motion in dispersed flow by Cumo et al [24] show drop velocities perpendicular to the wall similar to that predicted by Liu and Ilori. Their studies also show that drop velocities in the direction of flow are independent of drop diameter. Lee and Skinivasan [25] have shown a tendency for drop stratification in some cases of dispersed flow, however, no analysis is presently available which predicts this behavior. 
Ganic [26] has studied drop motion within the laminar sublayer of turbulent flow. His results show that the laminar sublayer has little effect on drops larger than about $10 \mu$. This is much smaller than average drop sizes found in dispersed flow, thus this effect may be ignored in analysis of this regime. 


\subsection{Objectives of Research}

In order to include the pertinent physical mechanisms in dispersed flow, the phenomenological approach was used in this investigation. The objectives were as follows:

1) To develop a new computer model based on the energy, momentum, and mass transport equations including recent correlations and analytic models which describe specific heat transfer mechanisms in dispersed flow.

2) To compare this computer model with world data.

3) To isolate specific areas where this model is deficient and more research is required.

4) To simplify portions of the numerical model until a closed form solution is obtainable without discarding physical relationships important to modeling the flow.

5) To compare this new closed form solution to world data. 


\subsection{Formation and Breakup of Liquid Droplets}

Dispersed flow is composed of droplets with differing diameters. Four significant mechanisms contribute to droplet sizing.

1. Helmholtz Instability - Droplet Formation

Vapor flowing over a liquid film ultimately produces waves on the surface, and entrains liquid in the form of droplets, Fig. 2-1a. Wicks and Duckler [27] measured drop sizes in unheated annular flow, using an electrical conduction technique. Their data, along with other annular flow data, was correlated by Tatterson et al [29]. Drop sizes were characterized by an upper limit log normal distribution, suggested by Muegele and Evans [28] for sprays.

Tatterson included the effect of vapor velocity by assuming drops are formed by the rupture of waves on the liquid film. A Kelvin-Helmholtz instability analysis resulted in a correlation for volume average diameter. 

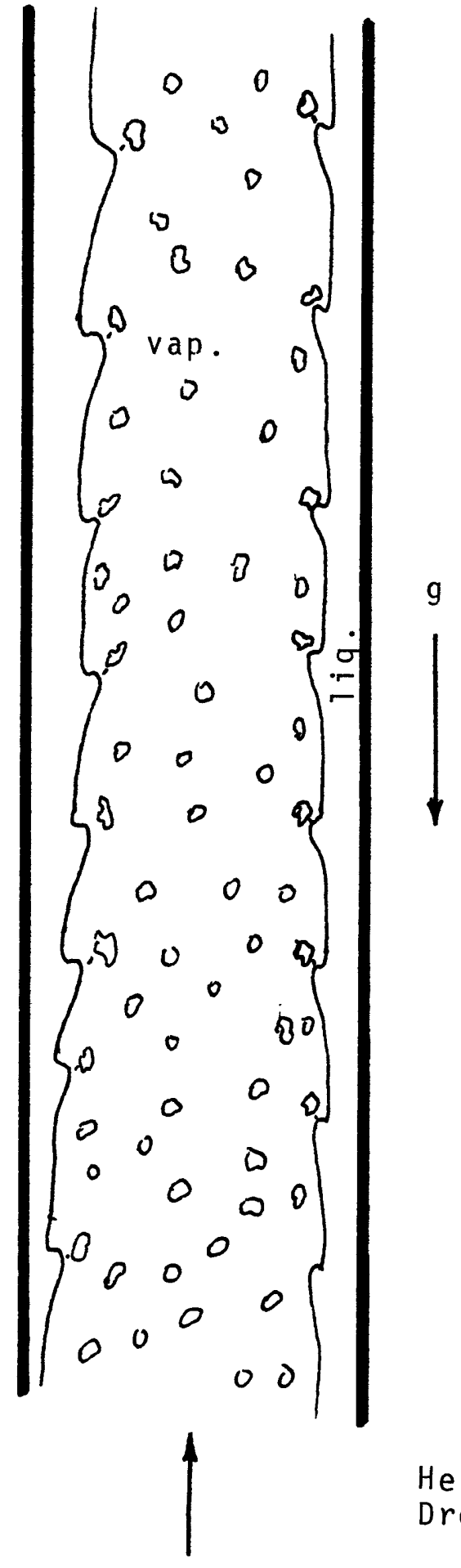

Helmholtz InstabilityDrop Formation

FIGURE 2-1a 


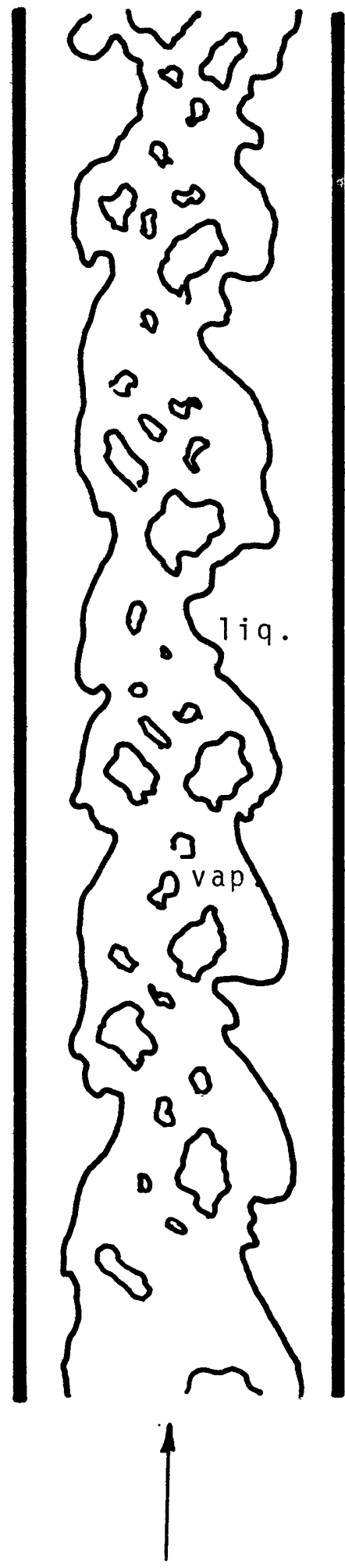

g

Film Slip Weber-

Drop Formation

FIGURE $2-1 b$ 
$-34-$

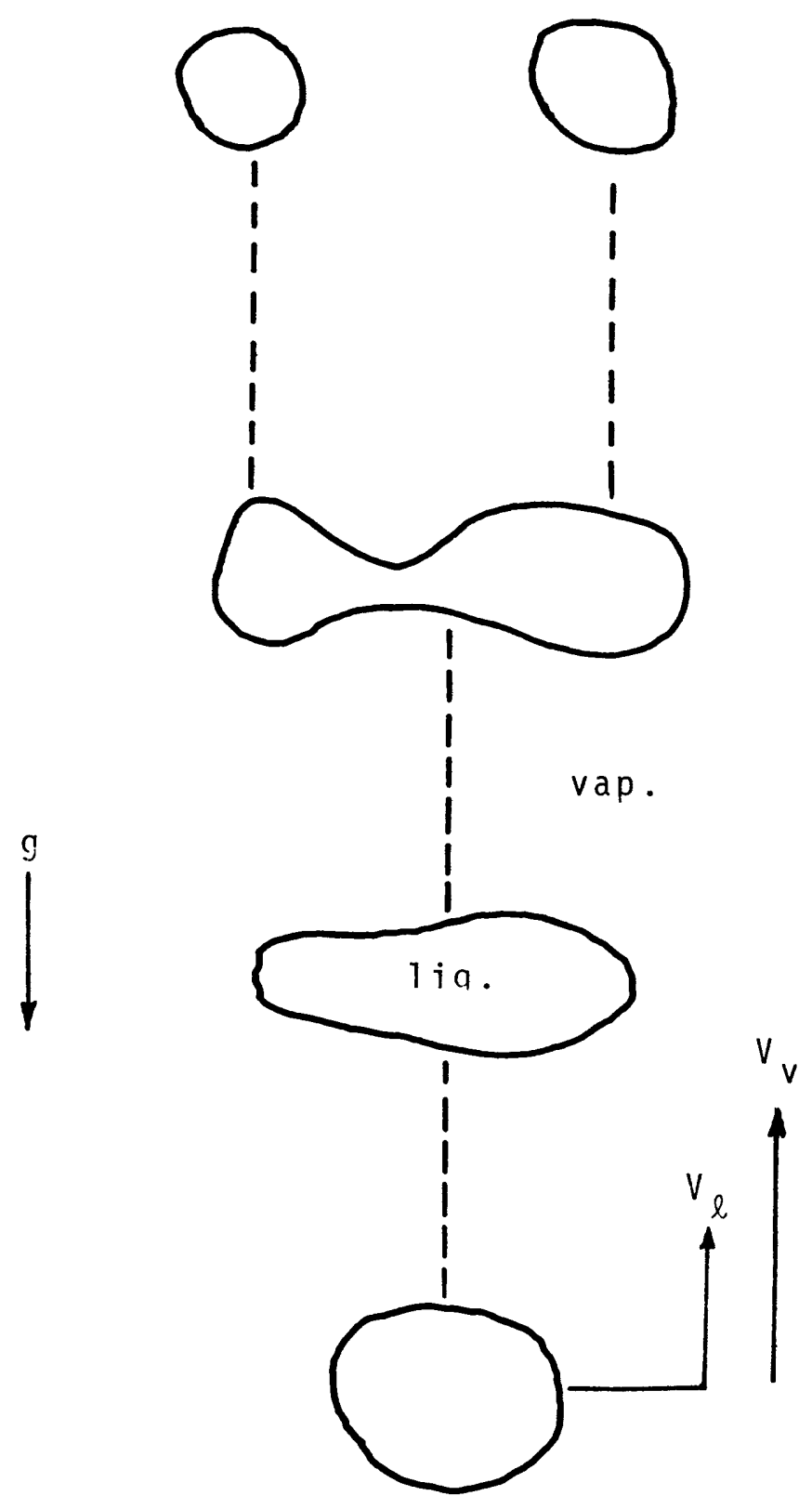

FIGURE 2-1c Weber Number Breakup 


$$
\frac{D}{D_{T}}=1.6 \times 10^{-2}\left(\frac{2 \sigma g_{c}}{\rho_{v} v_{v}{ }^{2} f D_{T}}\right)^{\frac{1}{2}}
$$

with the appropriate friction factor.

$$
f=\frac{.046}{\operatorname{Re}_{v} \cdot 2}
$$

where $\operatorname{Re}_{v}$ is the Reynolds number based on the vapor flowing alone in the channel.

2. Film Slip Weber Number - Droplet Formation

If vigorous boiling takes place in the channel, large chunks of liquid may be thrown into the stream, Fig. 2-1b. The relative velocity between the liquid film on the wall and vapor is then postulated to determine the drop size resulting from a critical Weber number criterion.

$$
w_{c}=\frac{\rho_{v}\left(v_{v}-v_{l}\right)^{2} D}{\sigma g_{c}}
$$

To predict drop diameter, $D$ from these equations, it is necessary to know We ${ }_{c}$ and $\left(v_{v}-V_{\ell}\right)$. Single drop measurements by Ishiki [30] suggested that the critical Weber number was 6.5. Later, magnitudes of 6.5 to. 7.5 were used in predicting heat transfer rates and drop sizes in 
dispersed flow $(3,18,5)$.

Ahmad [33] arrived at an expression for sip ratio based on void fraction data,

$$
S_{f}=\frac{V_{v}}{V_{\ell f}}=\left(\frac{\rho_{\ell}}{\rho_{v}}\right)^{.205}\left(\frac{G D_{T}}{\mu_{\ell}}\right)^{-0.016}
$$

Void fractions calculated using this correlation agree favorably with those calculated using the Dartmouth correlation and the separated flow model presented in Wallis [34] for annular flow, Fig. 2-2.

3. Free Stream Weber Number - Droplet Break-Up. Droplets entrained by the vapor experience drag as the vapor velocity increases due to heat addition and evaporation. Equation (2-3) may again be used with $\mathrm{We}_{\mathrm{c}}=$ 6.5 and $v_{\ell}$ equal to the free stream droplet velocity. Whenever the relative velocity produces a Weber number greater than 6.5 , it is assumed that the droplet breaks up reducing $W_{c}$ stepwise, Fig. 2-lc. For analytical purposes, we may hold the Weber number equal 6.5 and relate the drop diameter, D continuously with the relative velocity, $\left(v_{v}-v_{\ell}\right)^{2}$. 


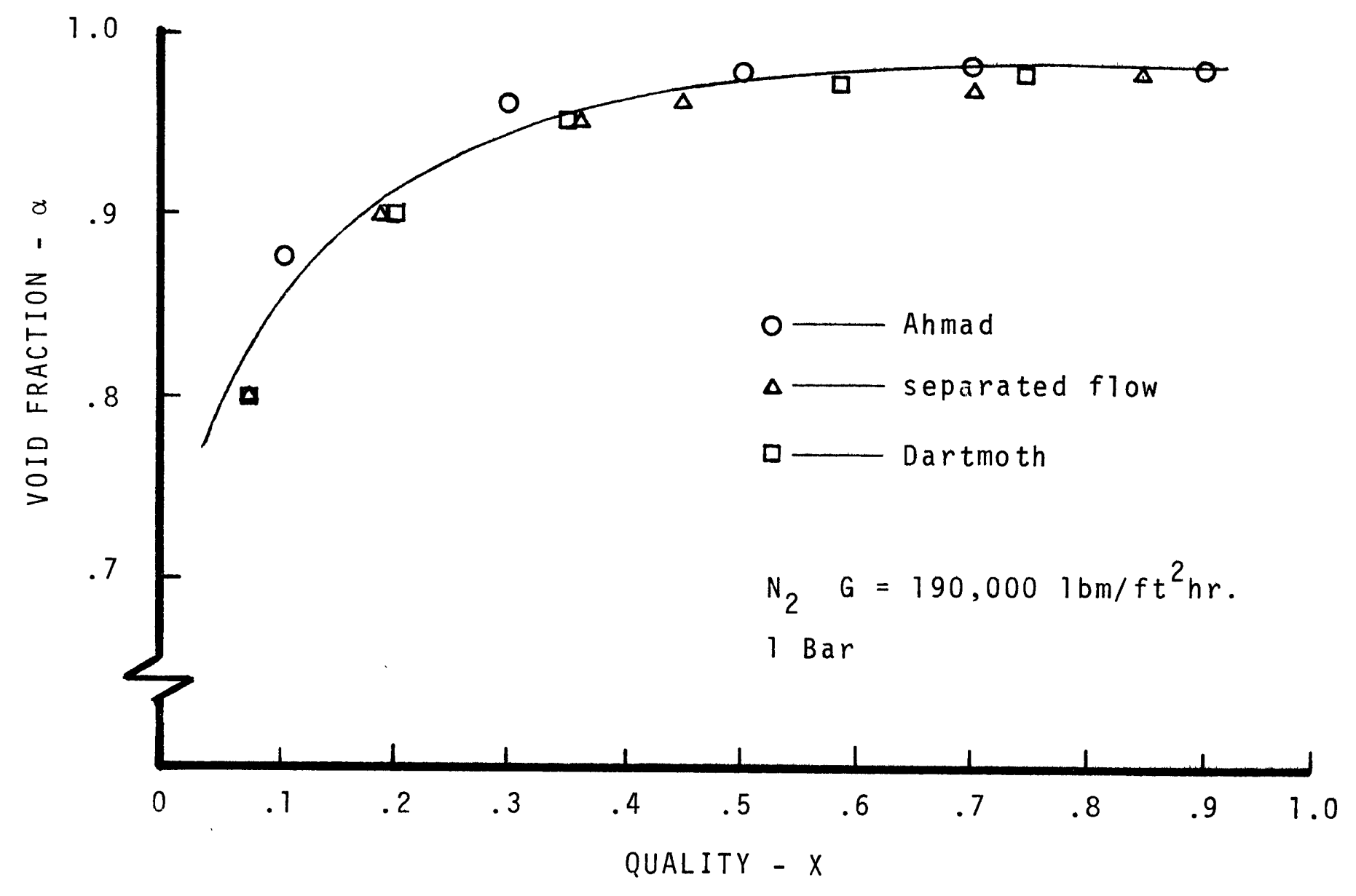

FIGURE 2-2 Void Fraction Comparison 


\section{Evaporation-Drop Size Decrease}

Downstream of burnout, non-equilibrium may exist in the flow, and heat can be transferred to the drops from superheated vapor in addition to that from the hot wall to the drops. Thus, droplet size decreases due to evaporation.

\section{1.a Inverted Annular Flow}

If the burnout quality is less than about $10 \%$, the flow regime is normally inverted annular. Visual studies in the M.I.T. Heat Transfer Laboratory show that drops break from the liquid core with a size the scale of the core diameter. Baum [31] investigated heat transfer during inverted annular flow. From flow pattern maps developed by Hosler [32], Baum concluded that inverted annular flow can exist only where void fractions are below $60 \%$. Thus, initial drop sizes would be about $80 \%$ of tube diameter.

Figure 2-3 shows drop formation in inverted annular flow. The large drops formed from the liquid core break up because surface tension forces are not strong enough to keep the drop intact in the presence of high relative velocities. A Weber number criterion should be important in determining initial drop size.

The definition of Weber number, Equation (2-3) can be combined with the definition of vapor and liquid 
$-39-$

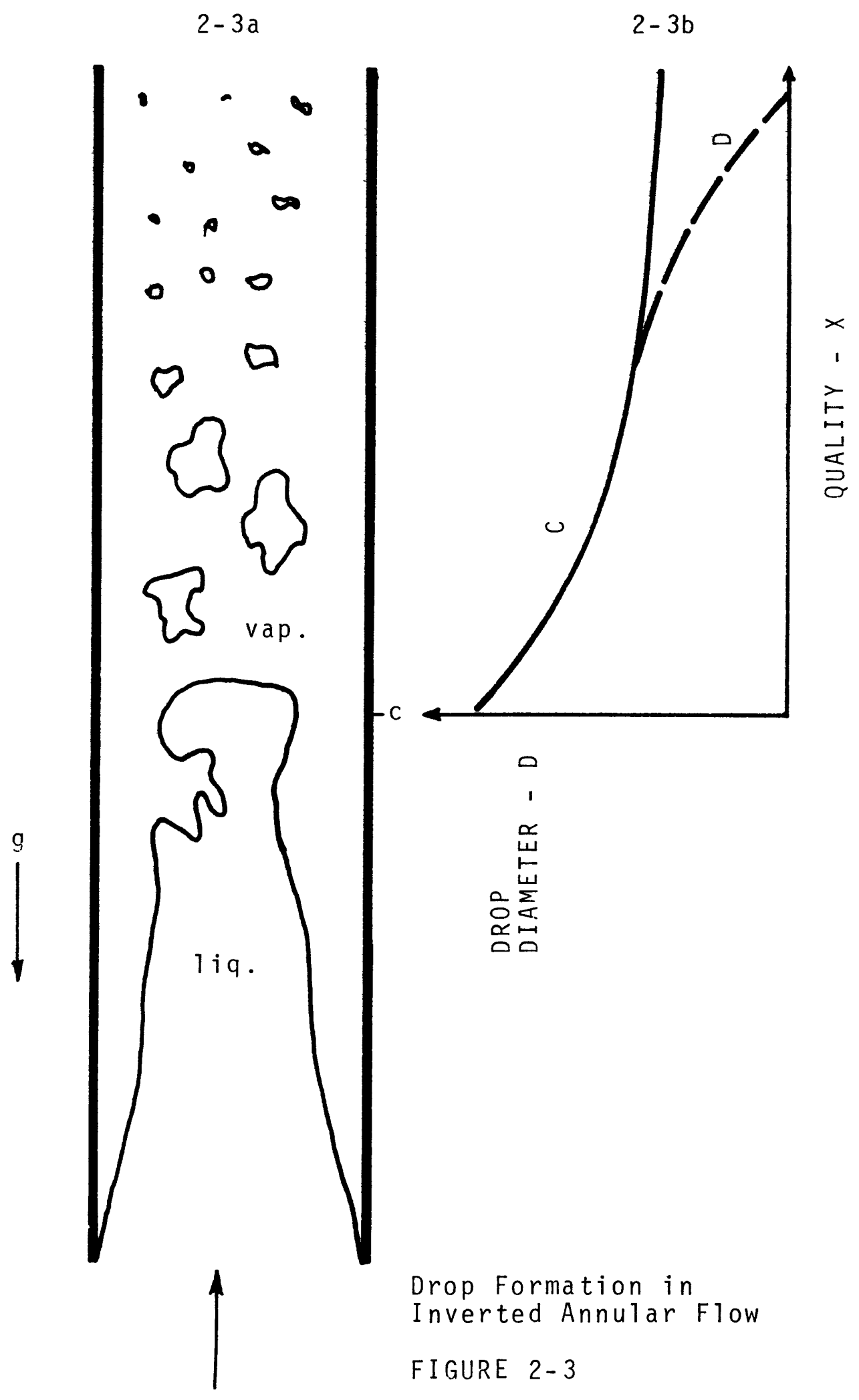


velocities,

$$
\begin{aligned}
& v_{v}=\frac{G X}{\rho_{v^{\alpha}}} \\
& v_{\ell}=\frac{v_{v}}{S}
\end{aligned}
$$

and the definition of void fraction

$$
\alpha=\frac{1}{\frac{\rho_{v}}{\rho_{\ell}} s \frac{1-x}{x}+1}
$$

to yield an equation for drop diameter as a function of quality and free stream slip at burnout.

$$
\frac{D}{D_{T}}=\frac{\rho_{v} \sigma{ }^{g} c}{G^{2} D_{T}} \quad \frac{W e_{c}}{(s-1)^{2}\left(\frac{\rho_{v}}{\rho_{l}}+\left(\frac{1}{S}-\frac{\rho_{v}}{\rho_{l}}\right) x\right)^{2}}
$$

As the drops travel downstream, they experience increasing relative velocity due to evaporation in the flow. The free stream Weber number could again be exceeded, and droplets would break up once more. Equation 2-8 could again be used to calculate a new drop diameter using local slip and quality (curve C, Fig. 2-3b).

Eventually evaporation of the drops control drop 
size (curve D, Fig. 2-3b) and no more break up occurs. This phenomenon is explained in more detail in section 2-1b under Annular Flow.

\section{$2.1 \mathrm{~b}$ Annu?ar Flow}

During annular flow, drops are formed at all points in the tube before burnout. Two types of sizing mechanisms may be important. The first is based on wave formation on the liquid film due to Helmholtz instability.

Tatterson's analysis was for unheated annular flows where the vapor velocity remains constant throughout the tube. In heated annular flow, the vapor velocity is constantly changing and an average drop diameter can be calculated from Equation (2-1) using the local vapor velocity. Thus, the characteristic drop diameter will decrease as quality increases. Equation (2-1) can be rewritten in terms of the quality and film slip using Equations (2-5) and $(2-7)$,

$$
\frac{D}{D_{T}}=.106\left(\frac{\sigma g_{C} \rho_{v}}{D_{T} G^{2}}\right)^{1 / 2}\left(\frac{G D_{T}}{\mu_{v}}\right)^{.1} \frac{X \cdot 1}{S_{f} \frac{\rho_{V}}{\rho_{l}}+\left(1-S_{f} \frac{\rho_{V}}{\rho_{l}}\right) X} \quad 2-9
$$

Thus, one characteristic drop size is associated with each point (or quality) before burnout. 
The variation of drop diameter due to Helmholtz instability, Equation (2-9) is shown sketched as a function of quality in Fig. 2-4 (curve A).

The film slip Weber number mechanism may also be important in determining the size of drops originating from the liquid film. The critical Weber number, Equation (2-5) and definitions of liquid velocity, Equation (2-6) and void fraction (assuming a film slip determined void fraction), Equation (2-7) can again be combined using the film slip, Equation (2-4)

$$
\left.\frac{D}{D_{T}}\right)_{f}=\frac{\rho_{v} \sigma g_{c}}{G^{2} D_{T}} \frac{W_{c}}{\left(S_{f}-1\right)^{2}\left(\frac{\rho_{v}}{\rho_{\ell}}+\left(\frac{1}{S_{f}}-\frac{\rho_{v}}{\rho_{\ell}}\right) x\right)^{2}}
$$

This is sketched as curve $B$ in Fig. 2-4.

The free stream slip Weber number mechanism determines the droplet diameter due to increasing relative velocity caused by the accelerating vapor stream. The drop diameter as a function of quality $X$ and local free stream slip velocity is given by Equation (2-8) and is sketched as curve $C$ on Fig. 2-4. Curve $C$ lies above curve $B$ because $\left(V_{v}-V_{\ell}\right)$ $<\left(v_{v}-v_{f}\right)$. Entrained drops with diameter lying above curve $C$ will always break up.

Because non-equilibrium exists in the flow after burnout, the drops evaporate as they travel down the tube 


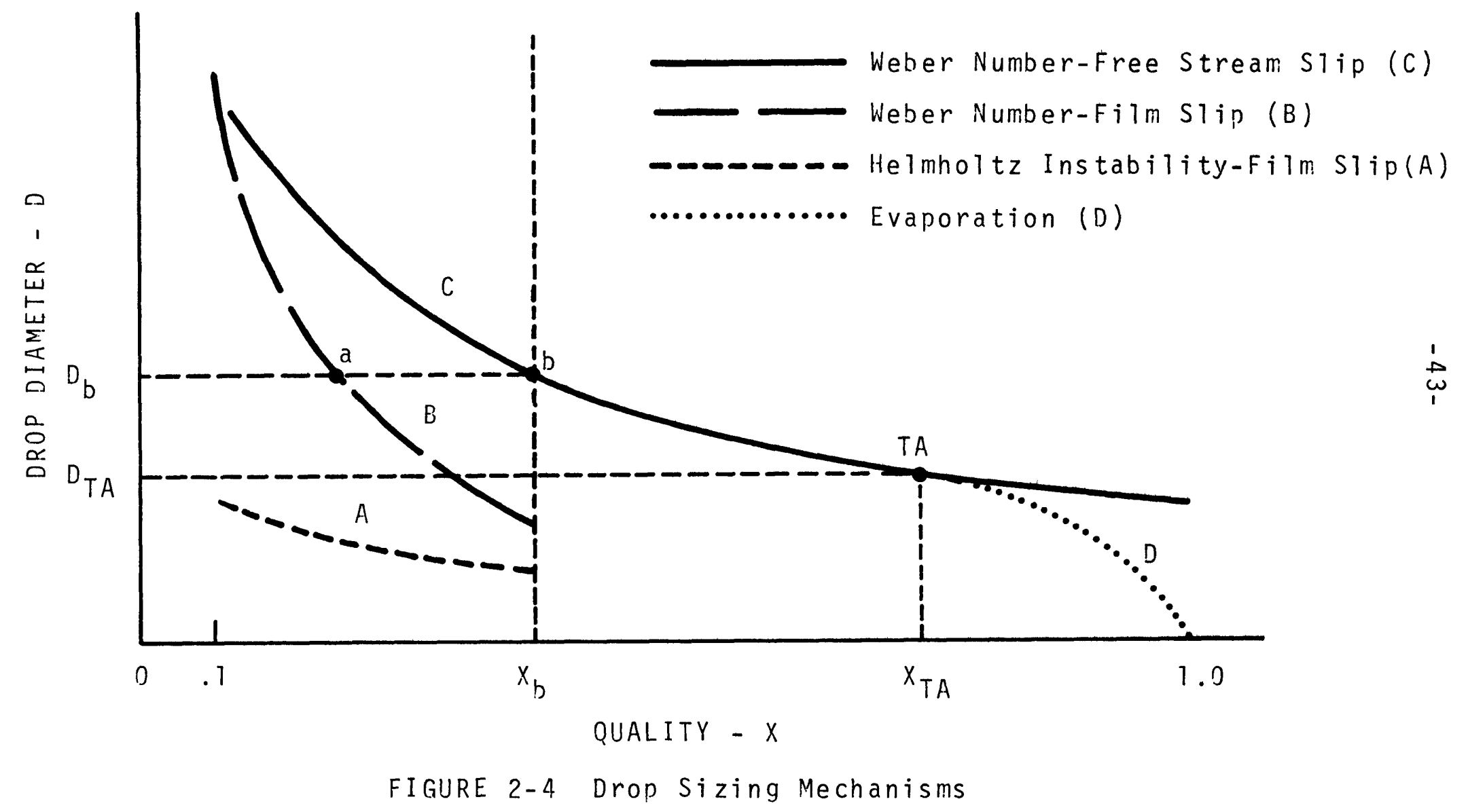


When the drop diameter gradient $\frac{d D}{d x}$ due to evaporation is larger than the diameter gradient due to the free stream critical Weber number (the derivative of equation 2-8), point TA of Fig. 2-4, break up no longer occurs and evaporation alone determines drop size. After breakup ends, the number of droplets in the flow remains constant. The variation of drop diameter with quality due to evaporation can be determined using a mass balance on the drops.

The liquid mass flow rate, $\dot{m}_{\ell}$ can be defined in terms of the drop number flux, $\dot{n}$,

$$
\dot{\mathrm{m}}_{\ell}=\rho_{\ell} \dot{\mathrm{n} \pi} \mathrm{D}^{3} / 6
$$

The definition of flowing quality,

$$
x=1-\dot{m}_{\ell} / \dot{m}
$$

can be combined with Equation (2-11) to give an equation for the quality in terms of drop diameter

$$
x=1-\rho_{\ell} \dot{n} \pi D^{3} / 6 \dot{m}
$$

For a constant droplet flux, 


$$
D \propto(1-x)^{\frac{1}{3}}
$$

and the drop diameter variation can be calculated.

$$
\frac{D}{D_{T A}}=\left(\frac{1-x}{1-x_{T A}}\right)^{\frac{1}{3}}
$$

This equation is curve $D$ in Fig. 2-4, where $\mathrm{X}_{T A}$ and $\mathrm{D}_{T A}$ are the quality and diameter at the tangent point of curves $D$ and $C$ (Equations (2-14) and (2-8)).

Figure $2-5$ is a plot of Equations $(2-8),(2-9),(2-10)$, and $(2-14)$ for Freon $12\left(G=486,940 \frac{1 \mathrm{bm}}{\mathrm{ft}^{2} \mathrm{hr}} ; Q=39371\right.$ $\left.\frac{\mathrm{Btu}}{\mathrm{ft}^{2} \mathrm{hr}}\right)$. In this case, the gradient of the evaporation curve (Equation (2-14)) is greater than the gradient of the Weber number curve (Equation (2-8)) at burnout. Thus, no breakup would occur after the burnout point.

Figure 2-6 shows various sizing sequences a drop may undergo after initial formation.

Any drops formed during annular flow larger than $D_{b}$ (Fig. 2-6), for example point $m$, follow a path m-nb-o-p with no diameter change between $m$ and $n$. Drops formed with diameters smaller than $D_{b}$, for example $D_{q}$ from the Helmholtz instability criterion, or $D_{r}$ from the 


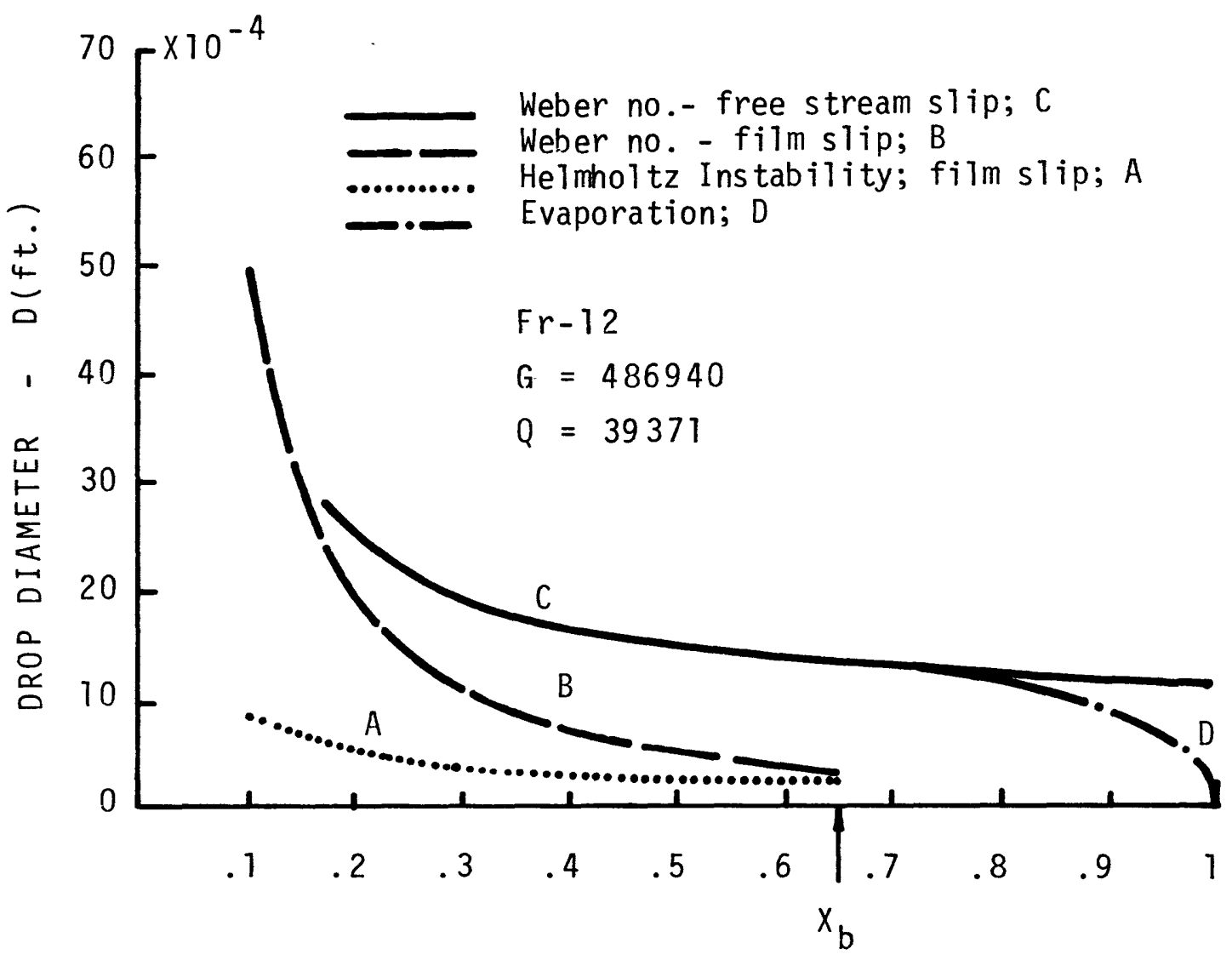

QUALITY - X

FIGURE 2-5 Drop Sizing Mechanisms - Fr-12 


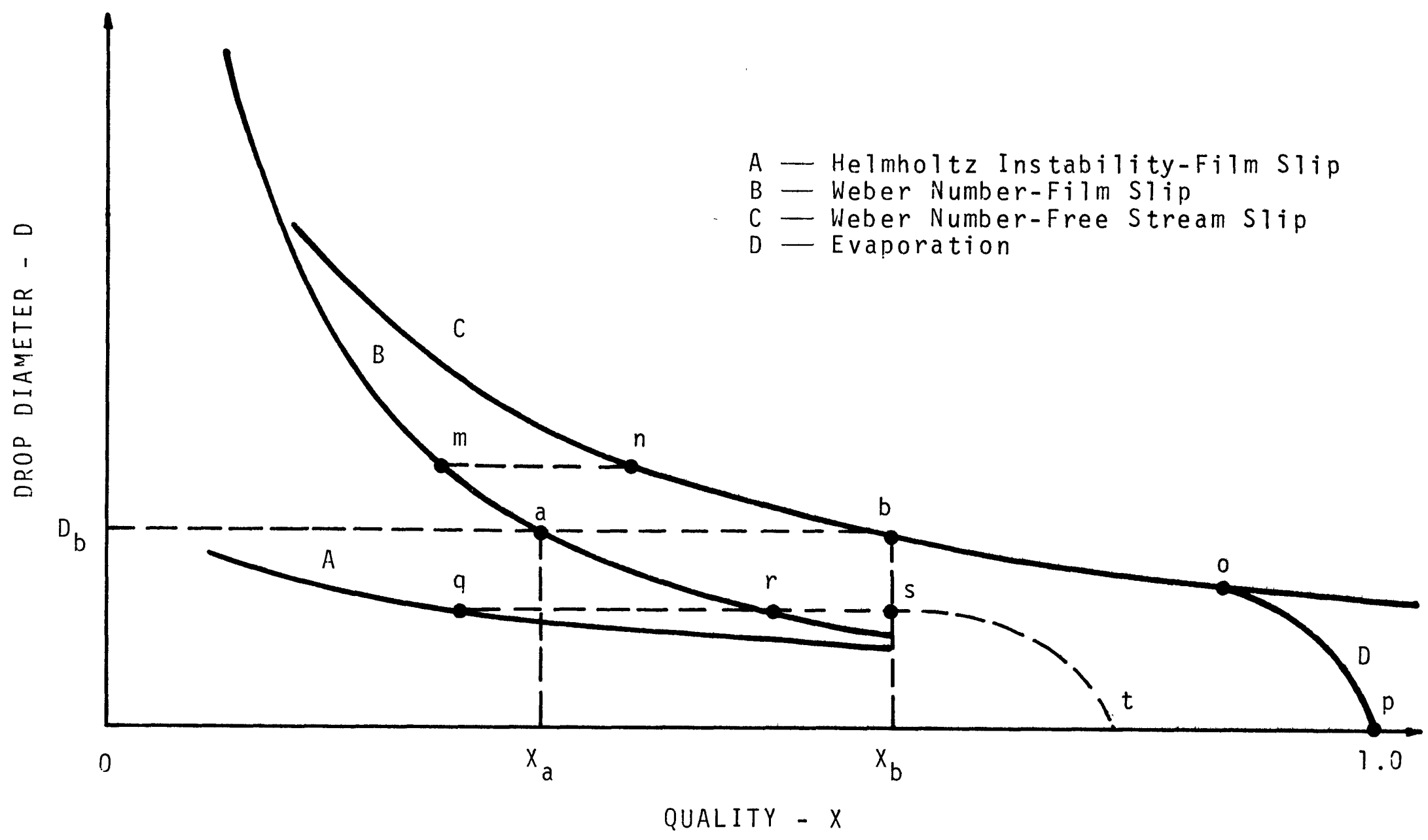

FIGURE 2-6 Drop Sizing Sequences 
film slip criterion, remain at the initial diameter uritil they reach the burnout point, $x_{b}$. After burnout, they decrease in size due to evaporation, along paths q-r-s-tor $r-s-t$.

\subsection{Average Drop Size at Burnout}

Most dispersed flow analyses use the concept of an average drop size to determine properties downstream of burnout. Drops are formed at points upstream of burnout, and the characteristic drop sizes are dependent on local conditions. It is necessary to find a method of averaging these various drop sizes in order to facilitate dispersed flow heat transfer calculations.

\section{2a Inverted Annular Flow}

Drops in inverted annular flow are formed after the burnout point. Initial average drop diameters for dispersed flow analyses should be based on the free stream Weber number criterion, Equation (2-8), at the point of liquid core breakup, for example point $C$ in rig. 2-1a.

\section{2b Annular Flow}

In order to average drop sizes for annular flow, all 
drops formed upstream of burnout must be considered. All drops originating upstream of point a (Figure 2-6) form with initial drop diameter $D>D_{b}$, due to Weber number breakup from the film. As they travel downstream, they break up by the free stream weber number criterion, to a diameter $D_{b}$ at the burnout point. Those drops that form from curves $B$ or $A$ with initial diameters less than $D_{b}$ remain at that size until they reach the burnout point. In order to determine the average drop size at burnout, the contribution of all of these drops must be considered. High speed photographic studies at the M.I.T. Heat Transfer Laboratory show that most entrained liquid forms from pieces of liquid being thrown from the film. The local drop size would best be characterized by the film sip Weber number mechanism. We therefore ignore the drop contribution from Helmholtz instability (Curve A, Fig. 2-6), Eq. 2-9).

In order to compute average drop diameters at burnout, it is necessary to know the cumulative mass distribution, E at the burnout point with respect to quality. The cumulative distribution is that amount of mass entrained previous to a quality $x$ which remains in the flow at burnout after accounting for deposition.

The mass entrained previous to point a (Fig. 2-6) which remains in the flow at burnout is $E_{a}$. All of this 
mass can be characterized by a drop diameter $D_{b}$. Between qualities $X_{a}$ and $X_{b}$, the entrained liquid remaining at burnout, $\left(E_{b}-E_{a}\right)$ will appear at diameters decreasing below $D_{b}$ as given by Equation (2-10). The mass weighted average diameter is then

$$
\frac{\bar{D}}{D_{T}}=\frac{1}{E_{b}}\left[E_{a} \frac{D_{b}}{D_{T}}+\int_{X_{a}}^{X_{b}} \frac{d}{D_{T}} \frac{d E}{d x} d x\right]
$$

We assume that the mass cumulative distribution, E varies linearly with quality, $X$ from $E=0$ at $X=0.1$ where annular flow is assumed to begin (this assumption is discussed more fully in Appendix $A-5)$.

$$
\frac{E}{E_{b}}=\frac{X-0.1}{X_{b}-0.1}
$$

Equation $(2-15)$ becomes

$$
\frac{D}{D_{T}}=\frac{x_{a}-0.1}{x_{b}-0.1} \frac{D_{b}}{D_{T}}+\int_{X_{a}}^{x_{b}} \frac{D}{D_{T}} \frac{d x}{x_{b}-0.1}
$$

By substituting Equation (2-10) into Equation(2-17) for $\bar{D}_{T}$ and integrating, the average drop diameter can be determined. 


$$
\begin{aligned}
& \frac{\bar{D}}{D_{T}}=\frac{1}{x_{b}-0.1}\left\{\frac{D_{b}}{D_{T}}\left(x_{a}-.1\right)+\left(\frac{\rho_{l}}{\rho_{v}}\right)^{2} \frac{W_{c}}{\left(S_{f}-1\right)^{2}} \frac{\rho_{v} \sigma g_{c}}{G^{2} D_{T}}\right. \\
& \left.\frac{1}{\left(\frac{\rho_{\ell}}{\rho_{v}} \frac{1}{S_{f}}-1\right)}\left[\frac{1}{1+x_{a}\left(\frac{\rho_{\ell}}{\rho_{v}} \frac{1}{S_{f}}-1\right)}-\frac{1}{1+x_{b}\left(\frac{\rho_{\ell}}{\rho_{v}} \frac{1}{S_{f}}-1\right)}\right]\right\} 2-18
\end{aligned}
$$

$\frac{D_{b}}{D_{T}}$ can be calculated using Equation (2-8) evaluated at burnout conditions.

The quality, $x_{a}$ where the film slip drop diameter equals the free stream slip drop diameter at burnout $\left(D_{b}\right)$ is obtained by equating drop diameters from Equations $(2-6)$ and $(2-8)$.

$$
\frac{\frac{\rho_{v}}{\rho_{\ell}}+\left(\frac{1}{s_{f}}-\frac{\rho_{v}}{\rho_{\ell}}\right) x_{a}}{\frac{\rho_{v}}{\rho_{\ell}}+\left(\frac{1}{s}-\frac{\rho_{v}}{\rho_{l}}\right) \times}=\frac{1}{s_{f}-1}
$$

If $x_{a}$ is less than $10 \%$, none of the drops formed in the annular region break up before reaching the burnout point. The drop size is determined solely by the film slip Weber number mechanism. The first term in Equation (2-18) should be eliminated, and $x_{a}$ replaced by 0.1 in the second term. 
CHAPTER III

\section{DISPERSED FLOW HEAT TRANSFER MODEL}

\subsection{Formulation}

The dispersed flow heat transfer model $\lfloor 1,2,3,5,18]$ consists of three of the heat transfer mechanisms shown in Fig. 3-1. A small portion of the tube wall is shown with vapor travelling with velocity $V_{v}$ and droplets travelling with axial velocity $V_{\ell}$. Because of turbulence and velocity gradients in the flow, the entrained drops move toward the wall with velocity $v_{p}$. Wall temperatures are high enough to prevent drop wetting, and approaching droplets ride on a vapor layer produced by rapid evaporation. The kinetic energy of the drop is absorbed by drop deformation and stored as surface energy. Surface forces try to restore the drop to a spherical shape, and the drop rebounds from the wall and travels downstream. Four heat transfer mechanisms can be identified from this representation.

1. Heat transfer directly from the wall to the vapor - Q 


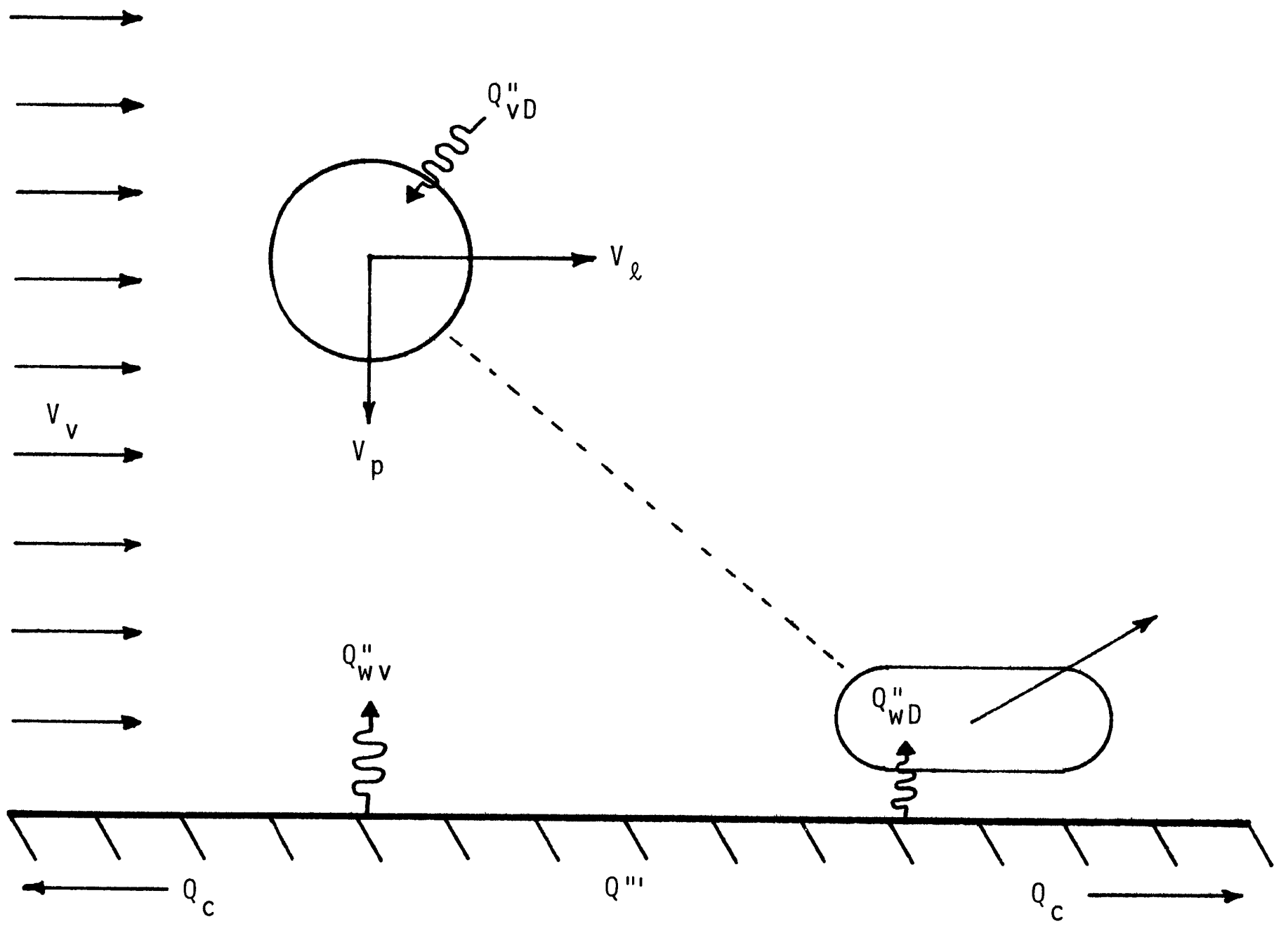

FIGURE 3-1 Heat Transfer Mechanisms in Dispersed Flow 
2. Heat Transfer from the Vapor to the Entrained Droplets - Q"

"Non equilibrium exists in the flow, and superheated vapor transfers heat to the drops.

3. Heat Transfer from the Wall to the Drops - Q ${ }_{W D}^{\prime \prime}$. Droplets in close proximity to the wall establish the local temperature profile, and therefore the local heat transfer.

Included in this analys is is one additional mechanism not contained in previous analyses.

4. Heat Transfer by Axial Conduction in the Tube $-Q_{C}$. High temperature gradients can exist in the tube wall near burnout and axial conduction could be impor$\tan t$.

The emphasis of this chapter is to develop a mechanistic heat transfer model which can predict tube wall temperatures in constant heat flux dispersed upflow. 


\section{ASSUMPTIONS}

Several assumptions are made which simplify the heat transfer model. These assumptions and their reasoning fol low:

1. The Flow is Steady State

Solution schemes have been compared to steady state experimental data. In some real world cases, this is a good approximation. For example, a once through steam generator operating under constant power conditions would be essentially a steady state process. 0 ther cases might not be steady state, however, could be considered "quasi" steady state. During the reflood portion of a nuclear reactor loss of coolant accident, cold water is used to cool fuel rods which are well above the Leidenfrost temperature. In this case, the burnout point or quench front velocities are on the order of inches per minute, and "quasi" steady state analysis should be appropriate. In other cases, where quench fronts move faster, as might occur in quench cooling of metals, the steady state assumption would not be appropriate, and a modification of this analysis would be necessary. 
2. The System is under Constant Heat Flux Conditions.

3. Equilibrium Quality Exists at Burnout.

Before the burnout point, vapor generated at the wall must travel through the liquid film, and little vapor superheat could exist. Therefore, the vapor and liquid temperatures must be equal at burnout.

4. The liquid is at saturation temperature at burnout and remains at the saturation temperature until completely evaporated.

5. The Drop Size Distribution can be Characterized by One Average Drop Size.

Drops of many sizes are present in dispersed flow. The assumption of a single drop size in the flow will cause a slight overprediction of equilibrium near actual qualities of 1 , since drops larger than the average drop size will exist in the flow, and will take longer to evaporate than a drop of average size.

Over the major portion of the flow pattern, the assumption of a single drop size would have little effect on the calculated wall temperatures, since the number of very large droplets existing in the flow is very small. 
6. The Wall Temperature at the Dryout Point is the Homogeneous Nucleation Temperature.

A liquid spontaneously boils at the homogeneous nucleation temperature. Any surface with temperature greater than the homogeneous nucleation temperature would prevent wetting. Previous to the burnout point, the liquid on the wall is in transition boiling. Once dryout occurs, liquid can no longer wet the wall. At this point, the wall temperature is thus assumed to be at the homogeneous nucleation temperature.

7. Liquid and Vapor Velocities are Uniform Across the Tube.

The vapor flow regimes present are turbulent, and vapor velocities would be nearly uniform. As stated earlier, experimental evidence shows that liquid drop velocities are approximately uniform across the tube and independent of drop size. 
KNOWNS AND UNKNOWNS

Knowns:

$\begin{array}{lll}X_{b} & - & \text { burnout quality } \\ G & - & \text { mass flux } \\ Q^{\prime \prime} & - & \text { heat flux }\end{array}$

Fluid Properties

Tube Properties and Dimensions

Unknowns:

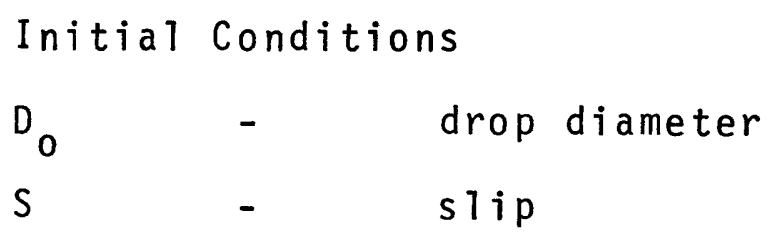

Local Conditions

$\begin{array}{lll}X & - & \text { quality } \\ D & - & \text { drop diameter } \\ V_{\ell} & - & \text { liquid velocity } \\ T_{V} & - & \text { vapor temperature } \\ T_{W} & - & \text { wall temperature }\end{array}$

$V_{v}, V_{\ell}$ and $\alpha$ can be calculated from Equations (2-5), (2-6) and (2-7) once $S$ is known. 
The model begins at burnout, where initial drop size is calculated by the procedure outlined in Chapter II, and the liquid velocity is calculated using the droplet momentum equation. The solution proceeds downstream using gradients of droplet diameter, actual quality, liquid velocity, vapor temperature, and an algebraic energy balance at the wall. Correlations are used for droplet heat transfer coefficient, droplet drag coefficient, and heat transfer coefficient between the wall and vapor. An analytic model is used to determine wall to droplet heat transfer.

\section{1 a Dryout Conditions}

The droplet momentum equation can be solved for free stream slip ratio at burnout, once the liquid acceleration is known. The liquid acceleration at burnout

$$
v_{\ell} \frac{d v_{\ell}}{d z}
$$

can be rewritten using the definition of liquid velocity Equation $(2-6)$. 


$$
V_{\ell} \frac{d V_{\ell}}{d z}=V_{\ell} \frac{G}{\rho_{V}} \frac{d\left(\frac{x_{e q}}{\alpha s}\right)}{d z}
$$

Under dispersed flow conditions, void fractions slightly less than one increase with distance while slip ratios, slightly greater than one decrease with distance. Therefore, at burnout, we assume that $x_{e q} \frac{d \frac{1}{s \alpha}}{d z} \ll \frac{1}{\alpha s} \frac{d x_{e q}}{d z}$ (see Appendix $A-1$ ).

The equilibrium quality at any point in the tube is,

$$
X_{e q}=\frac{4 Q^{\prime \prime}\left(z+z_{b}\right)}{G h_{f g} D_{T}}
$$

differentiating and substituting into Equation (3-1) with $x_{\text {eq }} \frac{d\left(\frac{1}{s \alpha}\right)}{d z}<\frac{1}{s \alpha} \frac{d x_{e q}}{d z}$,

$$
\frac{d V_{\ell}}{d z}=\frac{1}{s \alpha} \frac{4 Q^{\prime \prime}}{\rho_{V} h_{f g} D_{T}}
$$

The droplet momentum equation consists of droplet acceleration, the force due to gravity, and the drag on the drop. 
Acceleration Gravity Drag

$\frac{\pi D^{3}}{6} \rho_{\ell} v_{\ell} \frac{d v_{\ell}}{d z}=-\frac{\pi D^{3}}{6} g\left(\rho_{\ell}-\rho_{v}\right)+\frac{\pi D^{2}}{8} C_{D} \rho_{v} v_{\ell}^{2}(s-1)^{2} 3-4$

Substituting Equation (3-3) for $\frac{d v_{\ell}}{d z}$ and the definition of liquid velocity Equation (2-6), results in an equation for the free stream slip ratio.

$$
S=\frac{\left.1+\sqrt{1-\left(1-\frac{4}{3} \frac{\rho_{\ell} \rho_{V} g\left(1-\frac{\rho_{v}}{\rho_{\ell}}\right)}{G^{2} C_{D}} D \frac{\alpha^{2}}{x^{2}}\right)\left(1-\frac{16}{3} \frac{Q^{\prime \prime}}{G h_{f g}} \frac{D}{D_{T}} \frac{\rho_{\ell}}{\rho_{V}} \bar{x}\right.}\right)}{\left(1-\frac{4}{3} \frac{\rho_{\ell} \rho_{V} g\left(1-\frac{\rho_{V}}{\rho_{\ell}}\right)}{G^{2} C_{D}} D \frac{\alpha^{2}}{x^{2}}\right)}
$$

The definition of void fraction, Equation (2-7) and the free stream Weber number criterion, Equation (2-8) can be combined with Equation (3-5) in an iterative solution to solve for slip, void fraction and drop diameter at burnout.

\section{1b Governing Equations}

When modeling two phase flow, transport equations must be written for both phases. A set of equations which describe dispersed flow have been used by several investigators $[1-3,5]$. A good review of dispersed flow 
transport equations is given by crowe [36].

The equations presented here are those used by Forslund [3], Plummer [18] and Groeneveld [5].

\section{Liquid Velocity Gradient}

The droplet momentum balance Equation(3-4)

presented in section $3-1$ a is rewritten here

$$
\frac{d V_{\ell}}{d z}=-\frac{g}{V_{\ell}}\left(1-\frac{\rho_{v}}{\rho_{\ell}}\right)+\frac{3}{4} C_{D} \frac{\rho_{v}}{\rho_{\ell}} v_{\ell}(s-1)^{2} \frac{1}{D}
$$

where $C_{D}$ is the droplet drag coefficient.

2. Droplet Diameter Gradient

The liquid mass decreases due to droplet evaporation. An energy balance on the liquid includes heat transferred to the liquid from the superheated vapor - $Q_{V D}$, and heat transferred from the wall to the liquid- $Q_{W D}^{\prime \prime}$, Figure. (3-1). This in turn determines the rate of 1 iquid mass decrease.

$$
h_{f g} \frac{d m_{\ell}}{d t}=-\left[Q_{v D}^{\prime}+Q_{w D}^{\prime}\right]
$$

where $m_{\ell}, Q_{V D}^{\prime}, Q_{W D}^{\prime}$, are per unit length of tube. The liquid mass rate of change $\frac{d m_{\ell}}{d t}$ can be related to 
distance down the tube.

$$
\frac{d m_{\ell}}{d t}=v_{\ell} \frac{d m_{\ell}}{d z}
$$

For a unit length of tube, the relationship between the liquid mass and the drop diameter is:

$$
m_{\ell}=n \rho_{\ell} \frac{\pi D^{3}}{6} \frac{\pi D_{T}{ }^{2}}{4}
$$

where $n$ is the number of drops per unit volume. Or, using Equations (3-7) and $(3-8)$ and a constant drop number over $\mathrm{dz}$,

$$
\frac{d m_{\ell}}{d t}=\frac{1}{8} v_{\ell} n \rho_{\ell} \pi^{2} D_{T}^{2} D^{2} \frac{d D}{d z}
$$

VAPOR TO DROP HEAT TRANSFER - Q VD :

The heat transfer from the vapor to a single droplet is:

$$
q_{v D}=\pi D^{2} h_{D}\left(T_{v}-T_{s}\right)
$$


The heat transfer to all entrained droplets becomes: (again for a unit length of tube)

$$
Q_{V D}^{\prime}=n \frac{\pi D_{T}{ }^{2}}{4} q_{V D}
$$

or using Equation (3-10)

$$
Q_{V D}^{\prime}=\frac{1}{4} \pi^{2} D^{2} D_{T}^{2} h_{D}\left(T_{V}-T_{S}\right) n
$$

WALL TO DROP HEAT TRANSFER - $Q_{W D}^{\prime}:$

The heat transfer from the wall to a single droplet is :

$$
q_{W D}=\rho_{\ell} \frac{\pi D^{3}}{6} h_{f g} \varepsilon
$$

where the effectiveness, $\varepsilon$ is defined as the heat transfer to the drop divided by the total amount of heat needed to completely evaporate the drop.

The liquid mass flux toward the wall per length of tube is: 


$$
\dot{\mathrm{m}}_{\ell p}=\frac{1}{2} \rho_{\ell} V_{p} \pi D_{T}(1-\alpha)
$$

assuming half of the liquid is travelling toward the wall with velocity $V_{p}$ and half is travelling away from the wall.

The drop deposition rate (\#/hr ft) is $\dot{m}_{\ell p}$ divided by the mass per drop.

$$
\dot{n}_{p}=\frac{6 \dot{m}_{\ell p}}{\rho_{\ell} \pi D^{3}}=\frac{3 V_{P} D_{T}(1-\alpha)}{D^{3}}
$$

however, $(1-\alpha)=\frac{\text { Volume of Liquid }}{\text { Total Volume }}$

or $(1-\alpha)=\frac{\pi D^{3}}{6} n$

The wall to drop heat transfer is the product of the number of drops impacting per unit time and the heat transfer per impact. Combining Equations (3-12), (3-14) and $(3-15)$

$$
Q_{W D}^{\prime}=\frac{\pi^{2} D^{3} D_{T}}{12} h_{f g} V_{p} \rho_{\ell} n \varepsilon
$$

Placing Equations (3-9), (3-11a) and (3-16) into Equation (3-6) 


$$
\frac{d D}{d z}=-2\left[\frac{h_{D}\left(T_{V}-T_{s}\right)}{V_{\ell} \rho_{\ell} h_{f g}}+\frac{1}{3} \frac{D}{D_{T}} \frac{V_{p}}{V_{\ell}}\right]
$$

3. Actual Quality Gradient

The liquid flux, $G(1-x)$ can be written in terms of the axial droplet flux, $\dot{n}$.

$$
G(1-x)=\rho_{\ell} \frac{\dot{n} \pi D^{3}}{6} / \frac{\pi D_{T}{ }^{2}}{4}
$$

where $\dot{n}$ is the number of drops passing a cross section per time.

Differentiating with respect to $z$ and assuming the droplet flux is uniform across an element $d z$,

$$
\frac{d x}{d z}=-\frac{2 \rho_{\ell} \dot{n} \pi D^{2}}{G \pi D_{T}^{2}} \frac{d D}{d z}
$$

back substituting Equation (3-18) for $\dot{n}$.

$$
\frac{d x}{d z}=-\frac{3(1-x)}{D} \frac{d D}{d z}
$$

4. Vapor Temperature Gradient

The enthalpy increase in the vapor is the difference between total heat flux into the fluid minus that used to evaporate the liquid. 


$$
Q_{V}^{\prime}=Q_{T}^{\prime}-Q_{\ell}^{\prime}
$$

VAPOR ENTHALPY INCREASE: $Q_{V}{ }^{\prime}$

The vapor enthalpy increase across an element

$d z$ is:

$$
Q_{v}^{\prime}=\frac{1}{4} \pi D_{T}^{2} G \times C_{p} \frac{d T_{v}}{d z}
$$

The total heat transferred into the system is:

$$
Q_{T}^{\prime}=Q^{\prime \prime} \pi D_{T}
$$

LIQUID EVAPORATION: $Q_{\ell}$

The heat needed to evaporate the liquid and raise the resulting vapor to free stream temperature is:

$$
Q_{\ell}=\frac{1}{4}\left[C_{p}\left(T_{v}-T_{s}\right)+h_{f g}\right] G \pi D_{T}^{2} \frac{d x}{d z}
$$

combining Equations (3-21) through (3-24), 


$$
\frac{d T_{v}}{d z}=\frac{4 Q^{\prime}}{D_{T} G X C_{p}}-\left[\left(T_{v}-T_{s}\right)+\frac{h_{f g}}{C_{p}}\right] \frac{1}{x} \frac{d x}{d z}
$$

\section{Energy Balance at the Wall}

The heat into the fluid must all go into the drops impacting the wall $Q_{W D}^{\prime}$ or into the vapor $Q_{w V}^{\prime}$.

\section{VAPOR HEAT TRANSFER: Q ${ }^{\prime}$ '}

Heat transfer to the vapor/unit length:

$$
Q_{w V}^{\prime}=\alpha h_{w}\left(T_{w}-T_{v}\right) \pi D_{T}
$$

where $h_{w}$ is the wall to vapor heat transfer coefficient, and $\alpha$ is the void fraction which approximates the fraction of wall area free of drop interaction.

WALL TO DROP HEAT TRANSFER: $Q_{W D}^{\prime}$

$Q_{W D}$ is obtained is obtained by combining Equations $(3-15)$ and $(3-16)$

$$
Q_{W D}^{\prime}=\frac{1}{2}(1-\alpha) \pi D_{T} h_{f g} v_{p} \rho_{\ell} \varepsilon
$$


A non-linear temperature profile may exist under the drop, i.e. some heat from the wall may be used to superheat the vapor evaporated from the drop as well as evaporate the drop itself. A factor $\frac{\beta_{1}}{\beta_{2}}$ is included in the analysis to account for a non-linear temperature profile beneath the drop.

Thus, combining Equations (3-26) and (3-27) with the total energy into the system $Q_{T}^{\prime}$, Equation (3-23) and $\frac{\beta_{1}}{\beta_{2}}$.

$$
T_{w}-T_{v}=\frac{Q^{\prime \prime}}{h_{w}^{\alpha}}-\frac{1}{2} \frac{(1-\alpha) h_{f g} v_{p} \rho_{\ell}}{h_{w}^{\alpha}} \frac{\beta_{1}}{\beta_{2}} \varepsilon
$$

\section{3-1c Correlations}

Droplets in dispersed flow are exposed to an environment which may alter both the heat transfer and drag characteristics from that expected for simpler flows. Some of the more important effects are discussed below.

1. Droplet Heat Transfer Coefficient - Eq. (3-11a)

Droplet heat transfer coefficients may be affected by evaporation or free stream turbulence. 


\section{EFFECT OF EVAPORATION}

Mass transfer away from the drop has been found to decrease the heat transfer. Empirical shielding factors have been used to account for this effect. One common form which has been used by Ross et al [37] and Yuen et al [38] is:

$$
N u_{D}(1+C B)^{n}=N u_{0}
$$

where $\mathrm{Nu}_{0}$ is the Nusselt number for zero mass transfer, and $B$ is the spalding transport, or mass transfer number,

$$
B=\frac{C_{p}\left(T-T_{s}\right)}{h_{f g}-{ }^{q_{R} / \dot{m}}}
$$

$q_{R}$ is the radiative heat exchange, which was present in their experiment, and $\dot{m}$ is the mass transfer rate from the drop.

Ross experimentally investigated water drops evaporating in a steam environment. Drop Reynolds numbers were in the range of $30-200$ and the empirical shielding function which gave a satisfactory fit was

$$
(1+B)^{0.6}
$$


The zero mass transfer Nusselt number which Ross used was:

$$
\mathrm{Nu}_{0}=2+.369 \mathrm{Pr}{ }^{1 / 3} \mathrm{Re}^{.58}
$$

Yuen and Chen [38] chose a shielding function in the form

$$
N u_{D}(1+B)=N u_{0}
$$

to fit data of water and methanol evaporating in air for drop Reynolds numbers from 200 - 1000 and various free stream temperatures $\left(g_{R} / \dot{m}\right.$ was neglected in their analysis as it had little effect on the overall heat transfer). Yuen and Chen used the Ranz and Marshall correlation as the zero mass transfer Nusselt number.

$$
N u_{0}=2+.6 \operatorname{Re}_{f}^{1 / 2} \operatorname{Pr}_{f}^{1 / 3}
$$

Because the drop Reynolds numbers investigated by Yuen and Chen were closer to the range encountered in this analysis, and radiation to the drops is negligable (Appendix A-2), Yuen's shielding function 


$$
(1+B)=\left(1+\frac{C_{p}\left(T_{v}-T_{s}\right)}{h_{f g}}\right)
$$

and zero mass transfer number Equation (3-32) were chosen for use in Equation (3-31)

\section{EFFECT OF FREE STREAM TURBULENCE}

Drop heat transfer and drag correlations are based on the relative velocity between the drop and vapor. Drop Reynolds numbers in this study were found to be in the range where the boundary layer is laminar $(\approx 100<\operatorname{Re}<$ 1000). However, the free stream is turbulent, and turiulent fluctuations are present.

Hayward and Pei โ43」 experimentally examined the local heat transfer coefficient of a sphere in a stream with induced turbulence. The local Nusselt number ( $u_{D}$ as a function of position on the sphere), showed a dependence on the turbulence level in the flow, however, the overall Nusselt numbers were correlated well by solid sphere correlations. Reynolds numbers varied from 2500 to 6500 and turbulence intensity from . $5 \%$ to $5.7 \%$.

Maisel and Sherwood [45] studied the effect of free stream turbulence on mass transfer coefficients for 
spheres. Their experimental results show mass transfer coefficients increasing with increasing sphere Reynolds numbers and also with increasing turbulence intensity. However, low Reynolds numbers in combination with low turbulence levels had little effect on the mass transfer coefficient. They also found no effect due to the scale of turbulence in the flow.

Turbulence levels based on the relative velocity between the droplets and vapor are approximately $10 \%-20 \%$ in dispersed flow. For a turbulence level of $20 \%$, Maisel's data shows little increase in the mass transfer coefficient at a sphere Reynolds number of 2550.

Typical drop Reynolds numbers in dispersed flow range from 10 to 1000 over the major portion of the flow, therefore free stream turbulence is expected to have little effect on the heat transfer characteristics.

The heat transfer coefficient used in this study is that suggested by Yuen, Equations(3-31) and (3-32)

$$
N u_{D}=(1+B)^{-1}\left(2+.6 \operatorname{Re}_{f}^{1 / 2} \operatorname{Pr}_{f}^{1 / 3}\right)
$$


2. Drag Coefficient - Equations (3-4a), (3-4)

EFFECT OF EVADORATIOH!

Theoretical work by Hamielec [40] for low Reynolds number flows, indicated that droplet drag should decrease with increasing evaporation rates. However, experimental evidence presented by Yuen [41] suggests that evaporation has little effect on drag coefficients for mass transfer numbers, $B$, up to 3 . He interpreted the results by explaining that evaporation causes pressure drag to increase and friction drag to decrease, thus having little effect on total drag.

Ingebo [42] also noticed little difference in drag between evaporating droplets and non evaporating spheres. Mass transfer numbers in this study were always less than 3 , therefore the drag coefficient has not been altered because of evaporation.

\section{EFFECT OF ACCELERATION}

Droplets found in dispersed flow are typically 100 $200 \mu \mathrm{m}$. Because they are small, their acceleration is of the same order of magnitude as that of the vapor. Typical vapor accelerations range from 100 to $1,000 \mathrm{ft} / \mathrm{sec}^{2}$. Ingebo [42] found drag coefficients for accelerating liquid 
and solid spheres to be different from that for non accelerating spheres, other conditions being similar. The empirical expression which he suggested is:

$$
C_{D}=\frac{27}{\operatorname{Re}_{D}^{.84}}
$$

Accelerations in his experiments ranged from $6,000 \mathrm{ft} / \mathrm{sec}^{2}$ to $60,000 \mathrm{ft} / \mathrm{sec}^{2}$.

In analyzing dispersed flow, Groeneveld [5] used a minimum value of $C_{D}$ of .4 along with the Ingebo drag coefficient, while Hyrek [1] used a drag coefficient for a solid sphere.

$$
\begin{array}{ll}
C_{D}=\frac{24}{\operatorname{Re}_{D}\left(1+.142 \operatorname{Re}_{D} .698\right)} & \operatorname{Re}_{D}<2000 \\
C_{D}=.45 & \operatorname{Re}_{D}>2000
\end{array}
$$

Forslund [3] used a weighted average of the Ingebo $C_{D}$ and that of a solid sphere since drop accelerations encountered in his model were between Ingebo's experimental accelerations and gravitational acceleration (present in most steady state experiments). 
All of these investigations show an insensitivity to the choice of drag coefficient. Because accelerations are present in the flow, Groeneveld's technique was chosen for this analysis, Equation (3-34).

\section{EFFECT OF FREE STREAM TURBULENCE}

Free stream turbulence of the levels in dispersed flow has little effect on mass and heat transfer coefficients. Because these transfer mechanisms are similar to momentum transfer it is expected that free stream turbulence would have little effect on the drag coefficient.

3. Droplet Deposition Velocity-Eqs. (3-17), (3-18)

A droplet in dispersed flow experiences forces which tend to cause it to migrate perpendicular to the axial flow direction. A non-dimensional, deposition velocity, $\mathrm{V}^{+}$, was found to be a function of a dimensionless particle relaxation time, $\tau^{+}$, by Liu and Ilori [23].

$$
\begin{aligned}
& v^{+}=v_{p}\left(\tau_{0} / \rho_{v}\right)^{-1 / 2} \\
& \tau^{+}=2 / 9 \frac{\rho_{\ell}}{\rho_{v}} \frac{D^{2} \tau_{o} \rho_{v}}{\mu_{v}{ }^{2}}
\end{aligned}
$$


where $\tau_{0}$ is the shear stress at the wall. Figures 2 and 3 of their paper compare their calculations with deposition data. For values of $\tau^{+}$greater than 30 , the value of $\mathrm{V}^{+}$becomes constant. For example, for a $\operatorname{Re}$ of $10^{5}$, $\frac{\rho_{\ell}}{\rho_{V}}=11$, and $D_{T}=1 / 2$ in, $\tau^{+}$remains above 30 until drop diameters are less than $10 \mu$. Drop size in dispersed flow may begin at $100-200 \mu$ in diameter and evaporate completely. However, when the droplet evaporates to $10 \mu$, $99 \%$ of the mass has been evaporated, and the drop has 1 ittle effect on the flow for the remainder of its life. A constant value for $V^{+}$is therefore a good approximation. In this analysis, a value of .15 was used as suggested by Iloeje [4]. For a turbulent friction factor of $.046 / \mathrm{Re}^{.2}$, the drop deposition velocity, Equation (3-35), becomes

$$
\frac{v_{p}}{v_{v}}=.023 \mathrm{Re}^{-0.1}
$$

4. Drop-Wall Heat Transfer Effectiveness Equations $(3-17),(3-28)$

Forslund [3] discussed the role of drop-wall interactions in dispersed Flow. Hynek [1] used a drop-wall 
heat transfer coefficient based on information for single drops resting on a hot surface. Groeneveld [5] postulated dry wall collisions and treated the heat transfer as conduction across the vapor layer beneath the drop. Included in his analysis was an empirical determination of the appropriate vapor thickness. Iloeje [4] assumed that the drop came in intimate contact with the wall, and calculated appropriate heat up and nucleation times. The model assumed that some liquid remained attached to the heated surface after nucleation, and evaporated at the wall. By summing energies for heat up, nucleation, and evaporation he arrived at an expression for single drop heat transfer. Studies of individual drop impacts on a heated surface show a very low drop heat transfer effectiveness at dispersed flow film boiling temperatures $[19,22,46]$. Recently, McCarthy [22] has developed numerical and analytic models which account for deformation and vapor generation for heated, dry wall, drop impacts. The analysis was performed assuming a deformed droplet shape. The numerical analysis was carried out for two characteristic shapes. Both the circular cylinder and truncated sphere models showed proper trends and gave satisfactory agreement with water data. The analytic model was derived for the circular cylinder, and actually showed better data agreement than 
the numerical model. Her analytic model was chosen for this analysis because it includes the important physical parameters in dry wall impacts, and shows reasonable agreement with single drop data.

The equation for droplet heat transfer effectiveness is :

$$
\begin{gathered}
\varepsilon=2.6\left(\frac{\rho_{v}}{\rho_{l}}\right)^{1 / 2}\left(\frac{\bar{R}}{D}\right)\left(\frac{\rho_{l} v_{P}^{2} D}{\sigma g_{c}}\right)^{1 / 8} \cdot \\
\left(\frac{\beta_{2} k_{v}\left(T_{w}-T_{s}\right)}{h_{f g}\left(\rho_{v} \sigma g_{c} D\right)} 1 / 2\right)^{1 / 2}\left(I_{2} \frac{\mu_{v} h_{f g}}{\beta_{2} k_{v}\left(T_{w}-T_{s}\right)}+I_{1}\right)^{-1 / 4}
\end{gathered}
$$

$I_{1}$ and $I_{2}$ are constants with values of .225 and 1.5 respectively. $\bar{R}$ is average drop extension radius over the impact period.

$$
\bar{R}=\left(R_{\max }+.43 D\right) / 2
$$

$R_{\max }$ is the maximum extension radius, derived by equating the initial kinetic energy of the drop to the surface tension energy in the drop when it has come completely to rest. 


$$
\begin{array}{r}
\frac{R_{\max }^{2}}{D^{2}}=\frac{2}{3}\left(1+\frac{W e_{p}}{12}\right) \\
\cos ^{2}\left\{\frac{1}{3}\left[\arccos \left(\frac{-1.225}{\left(1+\frac{W e}{12}\right)^{3 / 2}}\right)\right]\right\}
\end{array}
$$

The Weber number $W_{p}$ used in this equation is defined using the initial inertia of the drop perpendicular to the wall.

$$
W e_{p}=\frac{\rho_{\ell} v_{p}{ }^{2} D}{\sigma g_{C}}
$$

Maximum extension radius calculated by Equation (3-39) is limited to $W e_{p}>1.74$. This arises from the assumption that the drop is a circular cylinder. Before deformation, the sphere and cylinder surface energies are not equal if the volumes are assumed equal, and an inital amount of $k i-$ netic energy is required to account for the difference. For We ${ }_{p}$ less than 1.74 it is assumed that $R_{\max }=.43 \mathrm{D}$, which is consistant with Equations(3-39) and (3- 38). $\beta_{2}$ accounts for the non linear temperature profile beneath the droplet. 


$$
\beta_{2}=\frac{1}{1+.3 \frac{C_{p}\left(T_{w}-T_{s}\right)}{h_{f g}}}
$$

In order to predict total heat transfer from the wall, the amount of heat used to superheat the vapor beneath the drop must be included. The ratio of the total to drop heat fluxes were determined from the temperature profile.

$$
\frac{q}{q_{D}}=\frac{\beta_{1}}{\beta_{2}}
$$

where $\beta_{1}$ is given as ${ }^{*}$ :

$$
\beta_{1}=\left(1+.43 \frac{C_{p}\left(T_{w}-T_{s}\right)}{h_{f g}}\right) /\left(1+.3 \frac{C_{p}\left(T_{w}-T_{s}\right)}{h_{f g}}\right) 3-43
$$

Equations (3-37) through (3-43) can be combined to calculate the drop heat transfer effectiveness.

McCarthy's analysis and most data apply to single drop impactions through a stagnant fluid. In the case of dispersed flow, a vapor temperature profile is already established at the wall due to forced convection. The The factors $\beta$ and $\beta$ have been corrected to these
values by McCarthy since publication of Ref. 22 . 
temperature gradient at the wall is established by the wallto-vapor Nusselt number.

$$
\left.\frac{d T}{d y}\right)_{v}=-\frac{1}{D_{T}} N u_{w}\left(T_{w}-T_{B}\right)
$$

The temperature gradient under the drop at the wall, predicted by the drop impaction analysis is defined by McCarthy using $\beta_{1}$.

$$
\left.\frac{d T}{d y}\right)_{D}=-\beta_{1} \frac{T_{w}-T_{S}}{\delta}
$$

where $\delta$ is the drop-wall separation distance, and $y$ is the distance from the wall.

$$
\begin{array}{r}
\delta^{4}=.45 \pi \bar{R}^{4}\left(\frac{1}{W_{p}}\right)^{1 / 2} \frac{\beta_{2} k_{v} \mu_{v}\left(T_{w}-T_{s}\right)}{\rho_{v} h_{f g} \sigma D g_{c}} \\
\left(I_{2}+\frac{\beta_{2} k_{v}\left(T_{w}-T_{s}\right)}{\mu_{v} h_{f g}} I_{1}\right)
\end{array}
$$

Two cases of drop impaction can be identified. The first case is where $\left.\left.\mid \frac{d T}{d y}\right)_{D}|>| \frac{d T}{d y}\right)_{V} \mid$. This case is depicted in Figure 3-2. The temperature profile beneath 

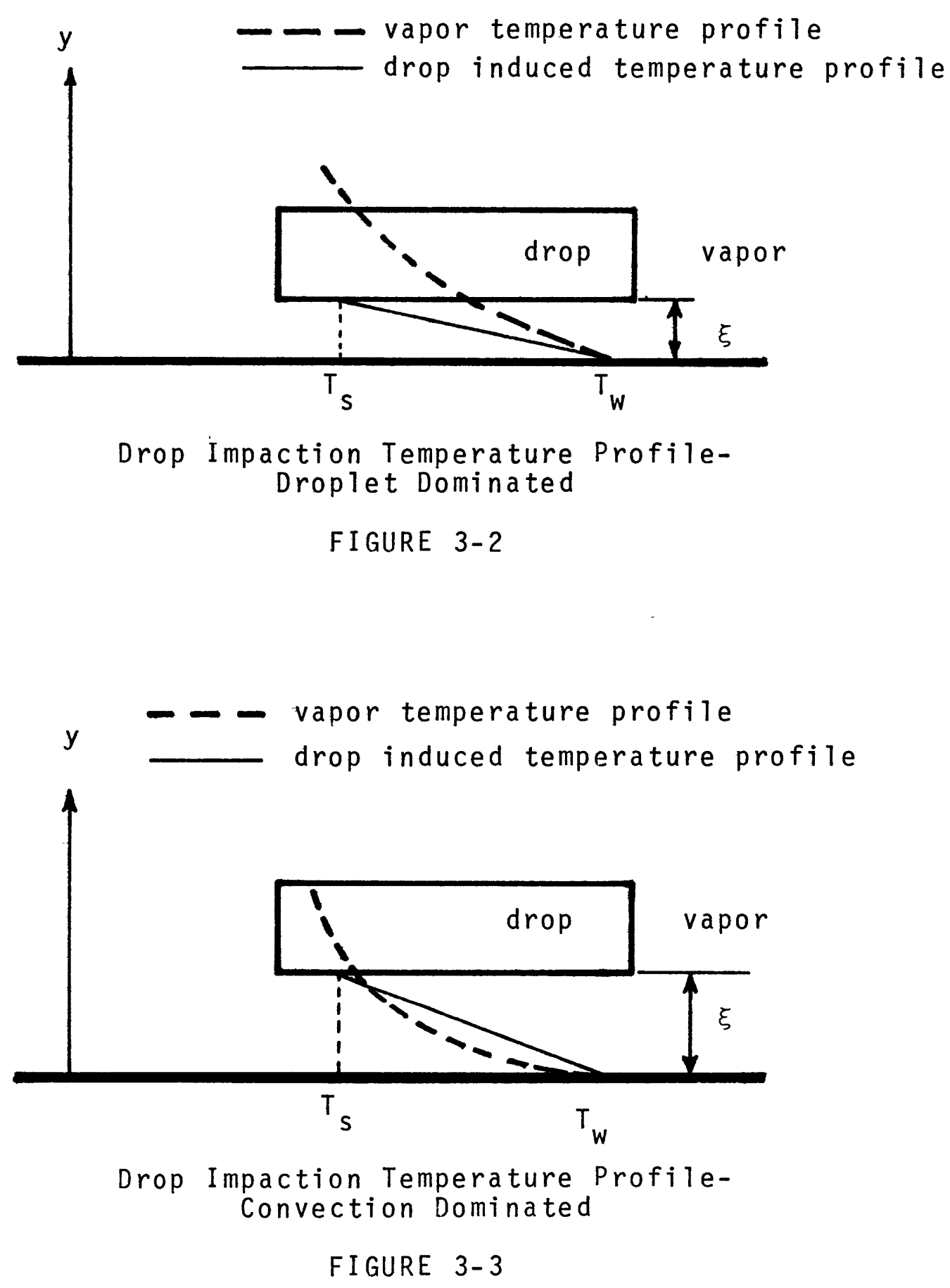
the drop is established due to the drop impaction and vapor dynamics beneath the drop.

$$
\text { The second case } \left.\left.\mid \frac{d T}{d y}\right)_{D}|<| \frac{d T}{d y}\right)_{V} \mid \text { is shown in }
$$

Figure 3-3. The drop has not penetrated the thermal boundary layer sufficiently to establish the temperature profile beneath the drop. In this case, the heat transfer would be determined by the forced convection temperature profile. The criteria for determining when this change occurs can be found by equating temperature gradients in Equations $(3-44)$ and $(3-45)$.

$$
\frac{\delta_{C}}{D_{T}}=\frac{\beta_{1}}{N u_{W}}\left[\frac{T_{W}-T_{S}}{T_{W}-T_{B}}\right]
$$

When the drop wall separation distance is greater than $\delta_{c}$ the convective temperature profile dominates. Drops at distances greater than $\delta_{c}$ would be expected to alter the convective temperature profile somewhat, however, to be conservative, the drop heat transfer term was included in the wall energy balance (Equation (3-28)) only when drop separation distances were less than $\delta_{c}$. The drop-wall interaction term in Equation (3-17) was included for all drop-wall interactions, since drops would still be in the high temperature wall layer. 
Property values used for calculating droplet effectiveness were based on the average temperature.

$$
T=\left(T_{w}+T_{s}\right) / 2
$$

5. Vapor Heat Transfer Coefficient Eq. (3-28)

The vapor heat transfer component in dispersed flow is normally modeled using single phase heat transfer correlations based on local conditions (i.e. using actual vapor velocities, temperature, etc.). One common form that has been used by many investigators $[1-3,5]$ follows the Dittus-Boelter form.

$$
N u_{w}=A \operatorname{Re}^{n} \operatorname{Pr}^{m}
$$

Several of these correlations and their applications are given in Table 3-1. For this investigation, the DittusBoelter equation with a vapor thermal entrance length from Kay's numerical solution [4] was used to calculate heat transfer coefficients for all fluids except water.

$$
N u_{W}=.023 \mathrm{Re}^{.8} \mathrm{Pr} .4\left(1+\frac{.8 D_{T}}{\mathrm{z}}\right)
$$

For water predictions, the Heinemansteam equation was used, Table 3-1. 
Forslund

$$
N u_{w}=.035 \mathrm{Re}^{.743} \mathrm{Pr}^{.4}
$$

Hynek (Dittus-Boelter) $\quad N u_{w}=.023 \mathrm{Re}^{.8} \mathrm{Pr} \cdot 4$

Koizumi

\begin{tabular}{|c|c|c|c|}
\hline $\begin{array}{l}\text { Bennett (Heineman) } \\
\text { Plummer }\end{array}$ & $\begin{array}{l}N u_{w}=.0157 \mathrm{Re}^{.84} \mathrm{Pr}^{1 / 3} \\
N u_{w}=.0133 \mathrm{Re}^{.84} \mathrm{Pr}^{1 / 3}\end{array}$ & $\left(\frac{D_{T}}{L_{D O}}\right)^{.04}$ & $\begin{array}{l}6<\frac{L_{D O}}{D_{T}}<60 \\
\frac{L_{D O}}{D_{T}}>60\end{array}$ \\
\hline
\end{tabular}

Groeneveld $\quad N u_{W}=.023 \mathrm{Re}^{.8} \mathrm{Pr}^{1 / 3}\left(\frac{\mu_{\mathrm{v}}}{\mu_{\mathrm{W}}}\right) \cdot 14,1+.3\left[\frac{\mathrm{D}_{\mathrm{T}}}{\mathrm{L}_{\mathrm{D} 0^{+} .01 \mathrm{D}_{\mathrm{T}}}}\right]^{.7}$
Plummer

$L_{D O}$ - length from dryout. Unless otherwise noted, properties are evaluated at free stream conditions

Table 3-1 
6. Conduction in the Tube Wall

High axial temperature gradients in the tube wall near burnout might lead to significant conduction effects. For the numerical solution, the tube wall was divided into axial segments as shown in Figure 3-4. $Q_{r}^{\prime \prime}$ is the heat flux into element $N$, and $Q^{\prime \prime}$ is the flux which leaves the inside tube wall, Equation (3-28). Temperature differences between the elements cause an axial heat flux within the wall. An elemental conduction equation can be written for a tube conductivity $k_{t}$ and thickness $t$

$$
Q^{\prime \prime}=Q_{r}^{\prime \prime}-\frac{2 k_{t} t}{\Delta z^{2}} T_{N}+\frac{k_{t} t}{\Delta z^{2}} T_{N-1}+\frac{k_{t} t}{\Delta z^{2}} T_{N+1}
$$

The heat flux Q" is composed of a flux to the vapor and a flux to the liquid drops.

$$
Q^{\prime \prime}=N u_{w} \frac{k_{v}}{D_{T}} \alpha T_{N}-N u_{w_{T}} \frac{k_{v}}{D_{T}} \alpha T_{v}+\frac{1}{2}(1-\alpha) v_{p} \rho_{\ell} h_{f g} \frac{\beta_{1}}{\beta_{2}} \varepsilon
$$

The conduction equation, [3-50] can then be written:

$$
\begin{aligned}
& -\frac{k_{t} t}{\Delta z^{2}} T_{N-1}+\left[N u_{W} \frac{k_{v}}{D_{T}} \alpha+\frac{2 k_{t} t}{\Delta z^{2}}\right] T_{N}-\frac{k_{t} t}{\Delta z^{2}} T_{N+1} \\
& =Q_{r}^{\prime \prime}+N u_{w} \frac{k_{v}}{D_{T}} \alpha T_{v}-\frac{1}{2}(1-\alpha) v_{p} \rho_{\ell} h_{f g} \frac{\beta_{l}}{\beta_{2}} \varepsilon
\end{aligned}
$$



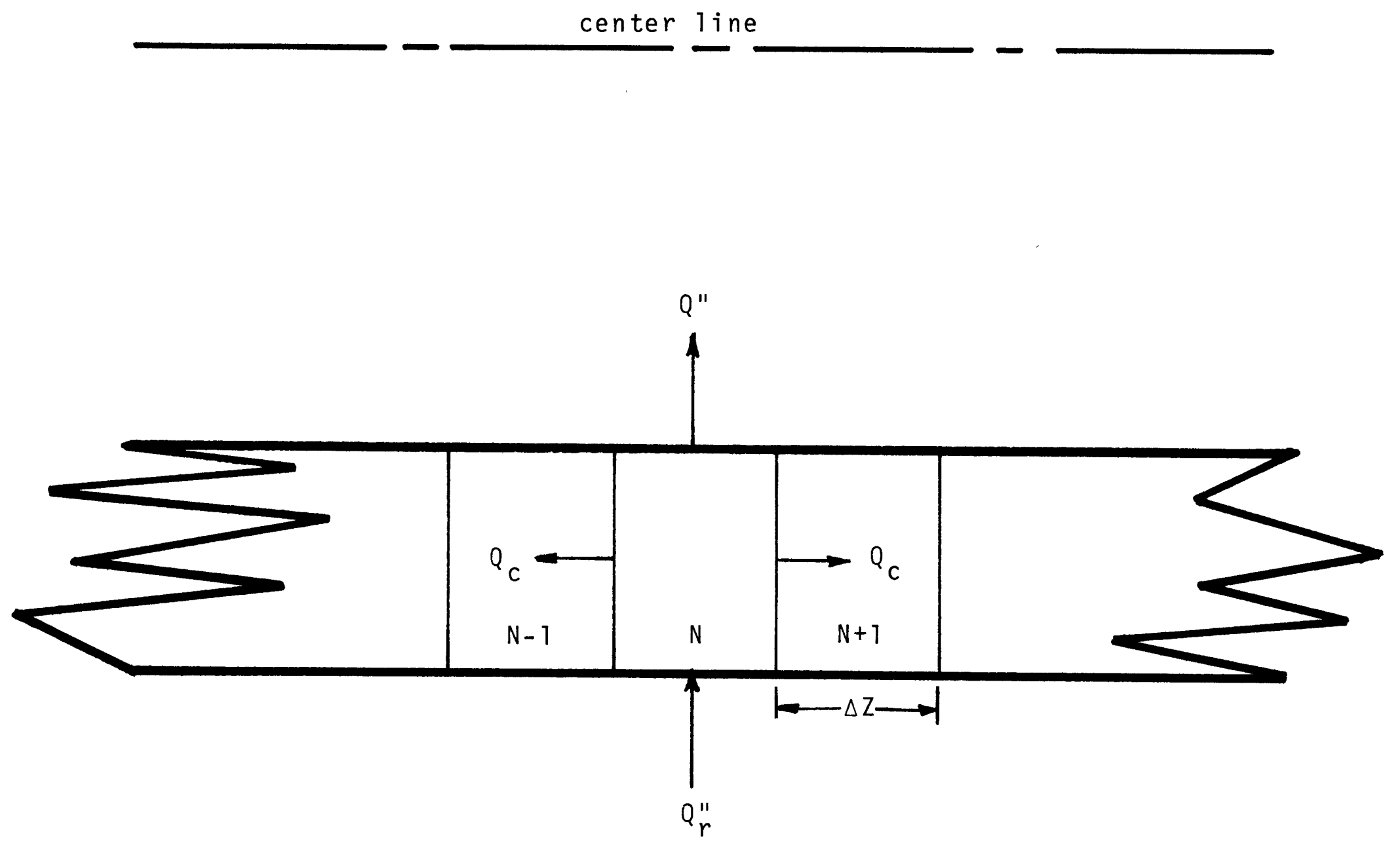

FIGURE 3-4 Axial Conduction in the Tube 


\subsection{Numerical Solution and Results}

The solution of Equations [3-4a], [3-17], [3-20], [3-25], [3-28] was performed using a fourth order RungaKutta integration scheme marching down the tube. Wall temperature at the burnout point was assumed to be the homogeneous nucleation temperature, while the last conduction element far downstream was assumed to be insulated from the remainder of the tube.

Initial drop size, void fraction, vapor velocity, and liquid velocitywere determined by using the procedure outlined in Section 3-1a. After the drops arrived at free stream conditions, if the Weber number, We, exceeded 6.5 at any point in the solution scheme, the drops were assumed to split into two drops of equal mass. Vapor properties were allowed to vary as a function of temperature, $\left(C_{p}, k_{v}, \rho_{v}, \mu_{v}\right)$.

General calculation procedure involved first marching down the tube and calculating wall temperatures and vapor properties at each axial conduction element without including the wall conduction. These wall temperatures were used as an initial guess so the local drop effectiveness, $\varepsilon$, and other quantities needed in Equation [3-51] could be calculated. A tri-diagonal conduction matrix was established using Equation [3-51] and local conditions. 
The matrix was solved for new wall temperatures using back substitution. These new wall temperatures were used to calculate the actual heat flux entering the fluid. The Runga-Kutta scheme was used again to calculate local fluid parameters. These in turn were used in another conduction matrix. Iteration was continued until the tube temperature profile converged. Never were more than three iterations needed before wall temperatures converged to within $1 \%$.

For most calculations, the step size and conduction element length were both $0.01 \mathrm{ft}$, however, if any instability in the solution scheme developed (most easily detected by looking at the relative velocity between the liquid and vapor, i.e. $v_{\ell}$ can not be greater than $v_{v}$ in upflow). The step size was automatically decreased by a factor of 10 until stability was achieved. Decreasing the step size to $.001 \mathrm{ft}$ had no effect on the calculated wall temperatures. 
3.2a Comparison of Numerical Model with Data FORSLUND'S NITROGEN DATA, [3]

Figure 3-5 and 3-6 compare the numerical solution scheme to Forslund's nitrogen data. Burnout was near the beginning of the tube and was probably inverted annular or froth flow as suggested by Plummer. Burnout qualities were assumed to be $1 \%$ which was always between the beginning of the tube and the first data point. Equilibrium quality was assumed to exist up to a quality of $6 \%$ as suggested by Forslund and Plummer's calculations to account for the non dispersed nature of the flow.

Near burnout, the model predicts wall temperatures slightly higher than the data. In all probability this is due to the type of flow regime present. The model assumes dispersed flow exists at the burnout point. If an inverted annular flow pattern is present, the slip ratio would be higher than the dispersed flow slip ratio. Vapor velocities would therefore be much higher, and the wall temperatures would be correspondingly lower.

The predicted wall temperatures agree well with the data at points downstream of burnout for almost all comparisons. However, the low heat flux data in Figure 2-6 lies above the predicted values. A low heat flux means that any heat losses (or gains) will have a significant effect 


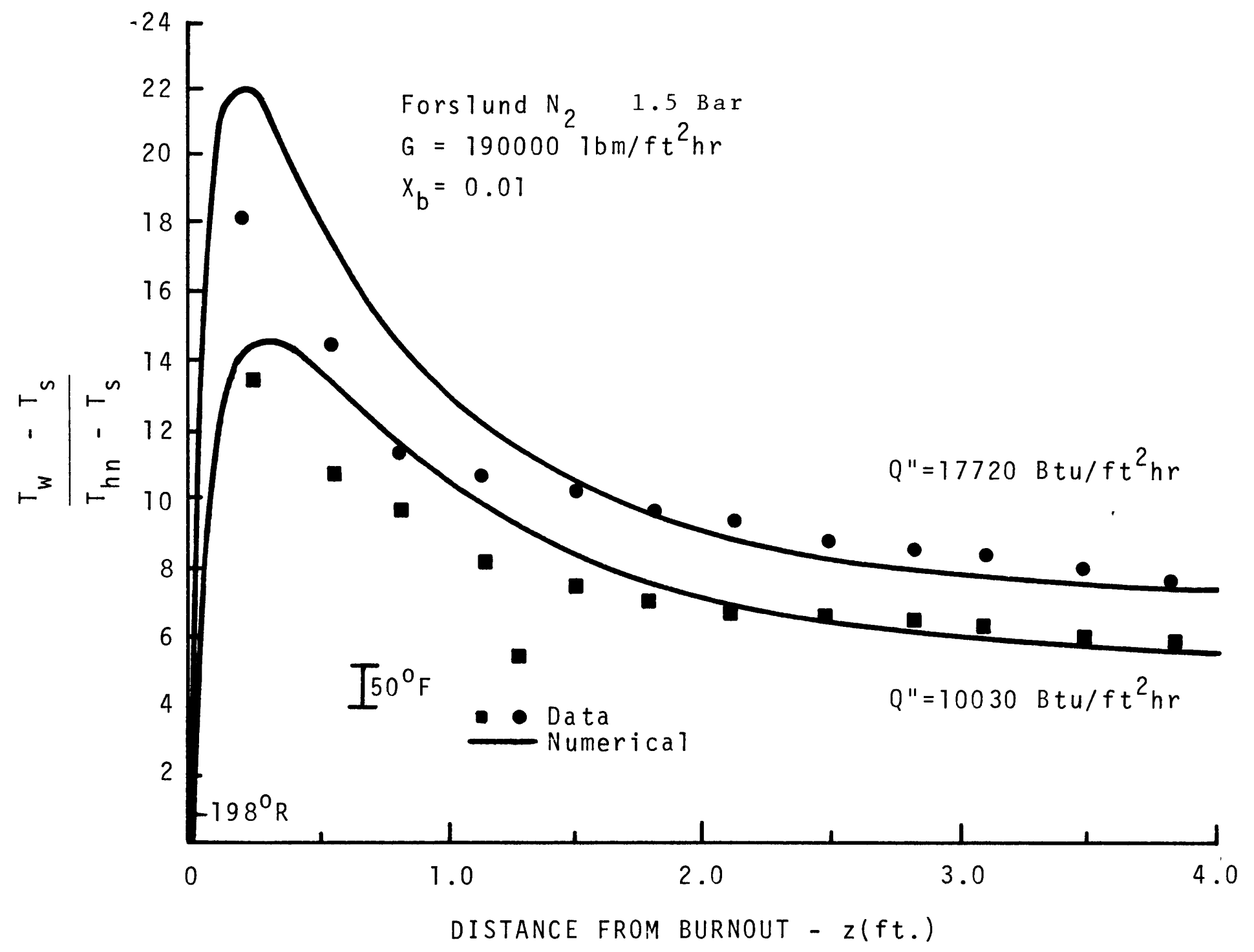

FIGURE 3-5 


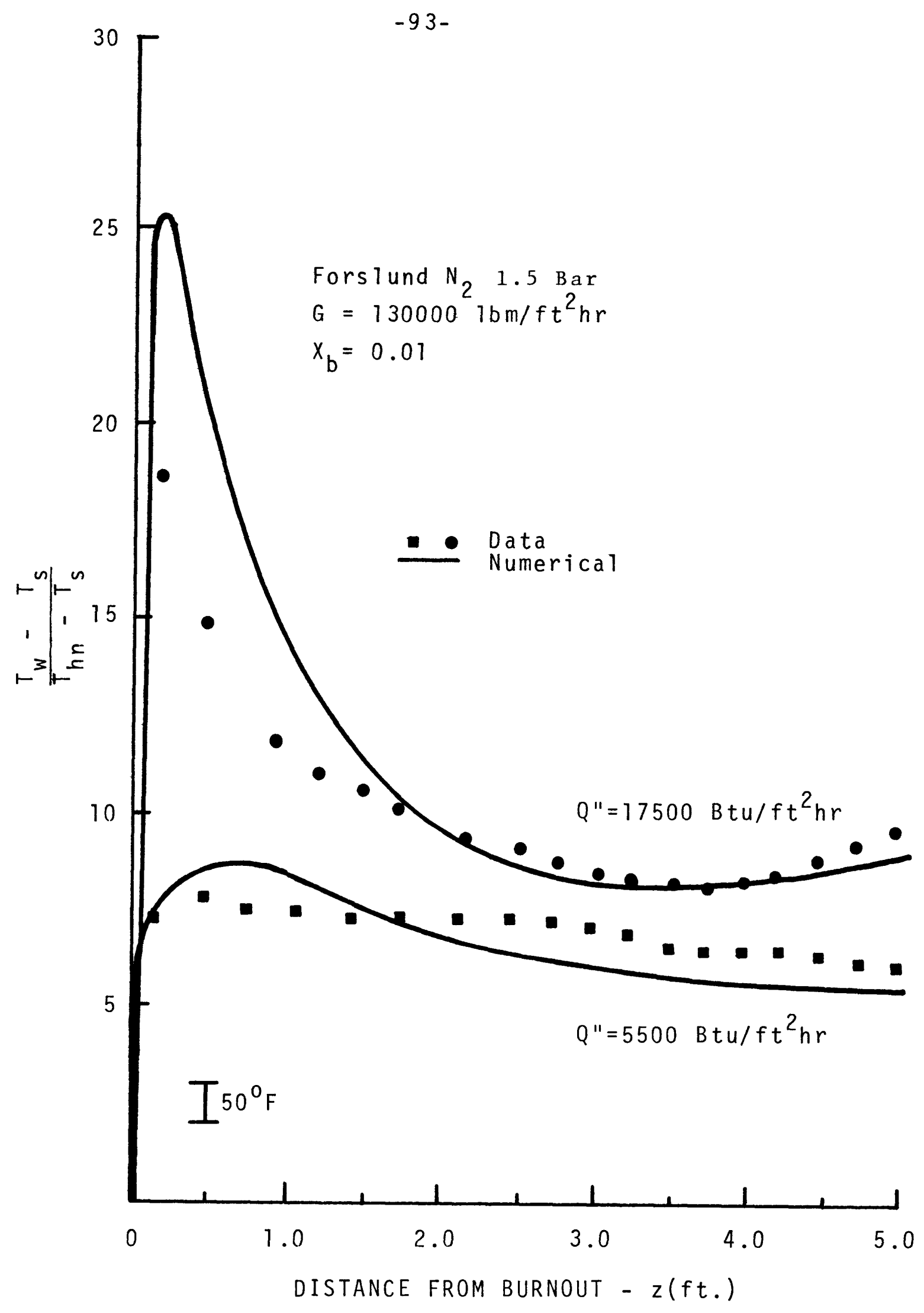

FIGURE 3-6 
on wall temperatures.

The wal1 temperatures in the $Q=5500 \frac{\mathrm{Btu}}{\mathrm{ft}^{2} \mathrm{hr}}$ run (Figure 2-6) are approximately $38^{\circ} \mathrm{F}$. Thus, heat gain can come from the atmosphere at room temperature. The natural convection heat transfer coefficient for a $\frac{1}{2}$ inch vertical tube at $38^{\circ} \mathrm{F}$ in a $70^{\circ} \mathrm{F}$ environment is approximately $2 \frac{\mathrm{Btu}}{\mathrm{ft} 2 \mathrm{hr}}$. this suggests that losses due to natural convection would account for less than $2 \%$ of the total heat flux in the tube if the tube were not insulated. However, at $38^{\circ} \mathrm{F}$, condensation of water vapor from the surrounding air would occur if the relative humidity were above approximately $35 \%\left(70^{\circ} \mathrm{F}\right.$ air temperature). Condensation heat transfer coefficients are much higher than natural convection heat transfer coefficients, and could cause the discrepancy in the results.

\section{GROENEVELD'S FREON - 12 DATA, [5]}

Comparison of the model to Groeneveld's Fr-12 data is shown in Figure 3-7. The model predicts data we11, however, some discrepancy between the data and the prediction scheme is apparent at points near burnout. Immediately downstream of burnout, a thermal boundary layer is beginning to develop. The model uses a thermal entry length for vapor alone. Drops present in the flow would have a 


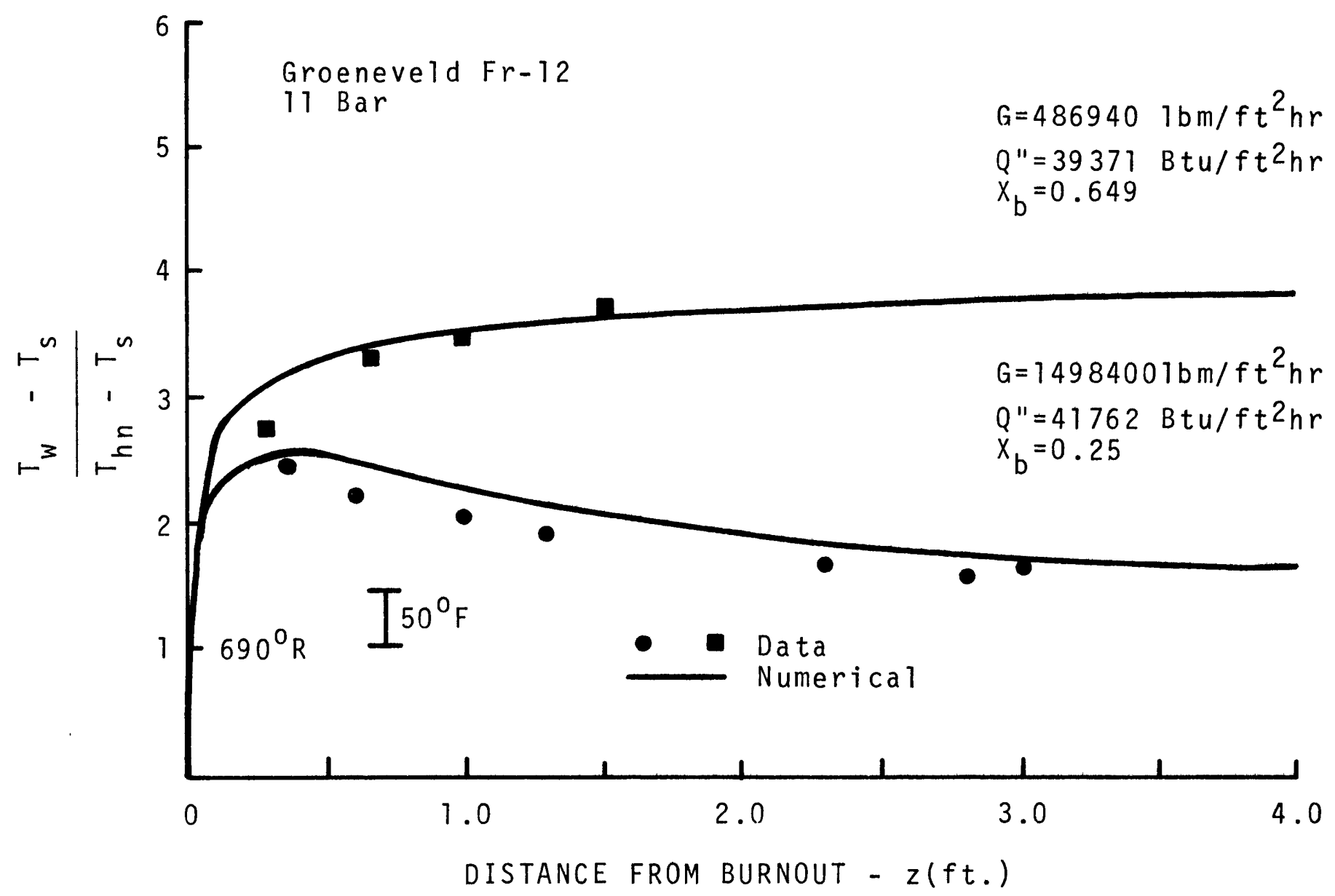

FIGURE 3-7 
tendency to increase the thermal entrance length by acting as a sink in the flow. Wall temperatures would therefore be lower than those predicted using a vapor entrance length alone. A further discussion of this phenomenon is presented in Appendix A-3.

BENNETT'S WATER DATA, [2]

Figures 3-8 and 3-9 compare the model with Bennett's water data. Predicted wall temperatures agree with the data to within about $50^{\circ} \mathrm{F}$. The shapes of the predicted curves all seem to agree with the data, however, the magnitude of the predicted temperatures for $G=3.8 \times 10^{6} \mathrm{lbm} /$ $\mathrm{ft}^{2} \mathrm{hr}$ run (Figure 3-9) are slightly above actual wall temperatures. Agreement might be better if a wall-tovapor heat transfer coefficient higher than that predicted by the Heineman steam equation is used. Figure $3-10$ is a plot of the $G=3.8 \times 10^{6} \mathrm{lbm} / \mathrm{ft}^{2} \mathrm{hr}$ run using two different heat transfer coefficients. The upper curve shows predicted wall temperatures using the Heineman equation and is the same curve as in Figure 3-9. The lower curve shows predicted wall temperatures using the Dittus-Boelter equation. Temperatures predicted by the Dittus-Boelter equation are too low, primarily because the Heineman equation is based on flim properties, while the Dittus-Boelter equation is based on bulk properties. However it does show the effect 


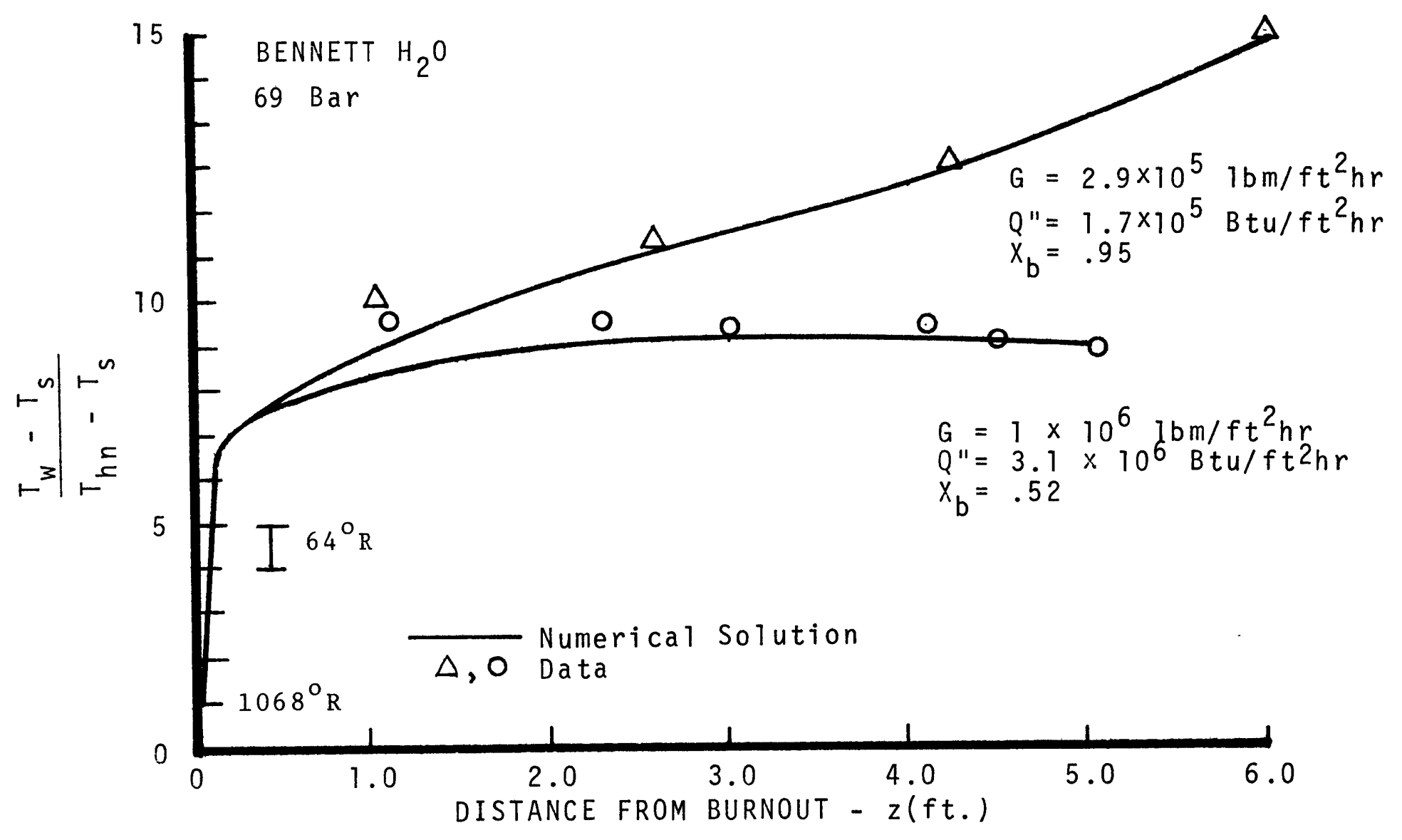

FIGURE 3-8 


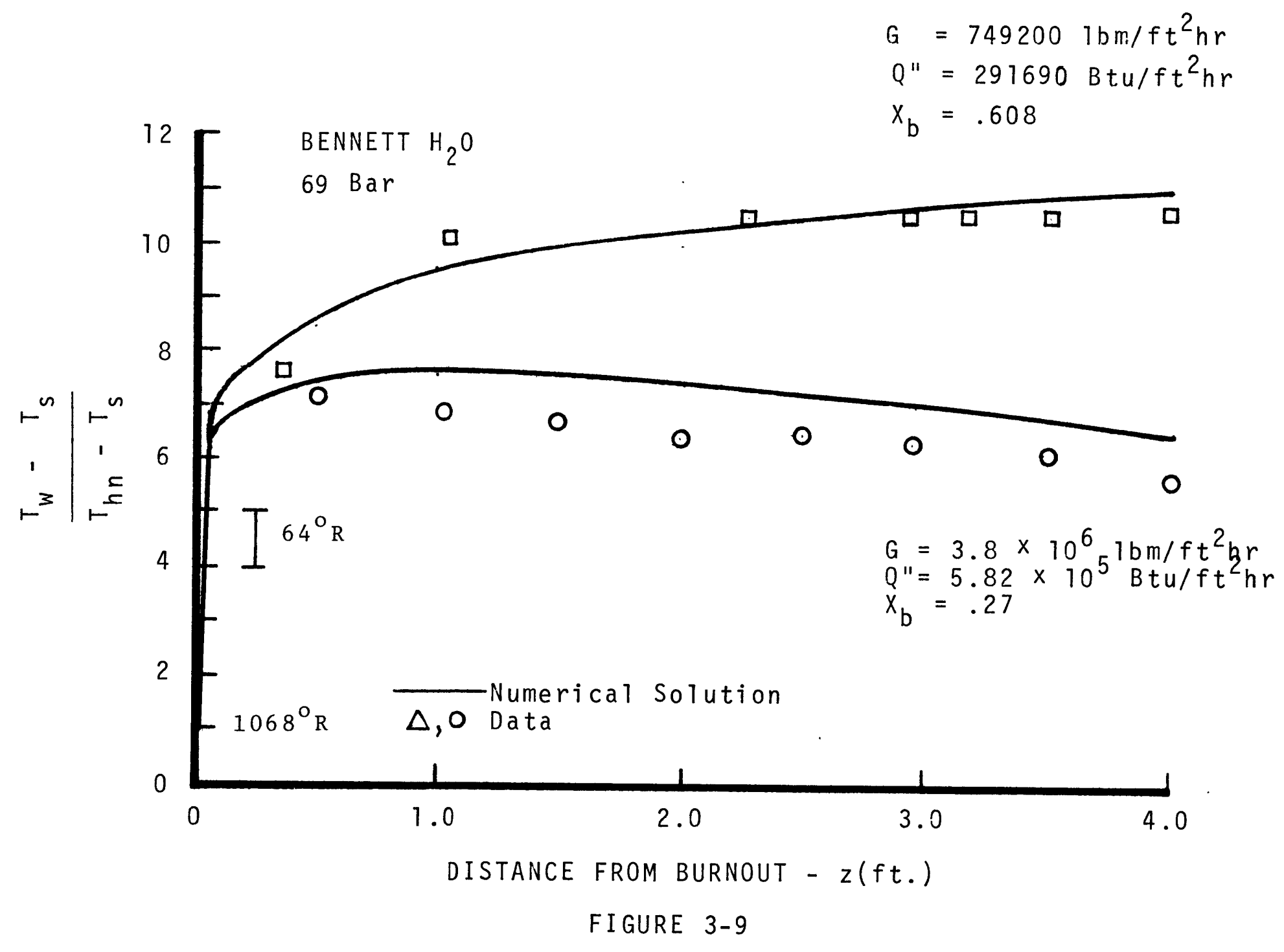




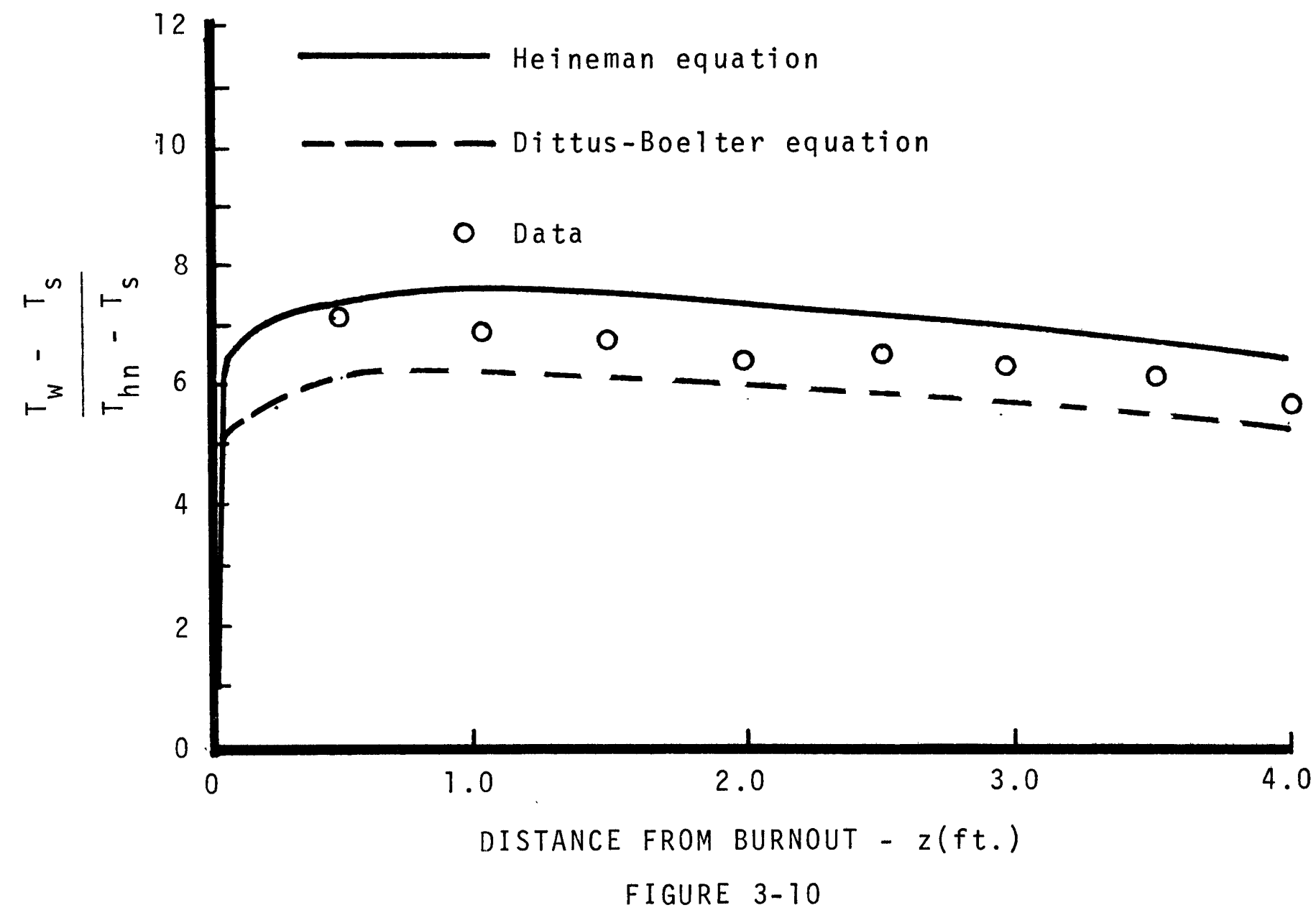


of a higher heat transfer coefficient on predicted wall temperatures.

$$
\begin{aligned}
& \text { CUMO'S FREON-12 DATA, [6] } \\
& \text { Predictions of Cumo's Freon-12 data is much poorer }
\end{aligned}
$$
than that for the other fluids. Figures 3-11 and 3-12 compare the prediction scheme with Cumo's data. Compared to predicted wall temperatures, the general shape of the data seems to suggest that more equilibrium exists in the flow than the model predicts. However, Figure 3-13 shows the $G=7661401 \mathrm{bm} / \mathrm{ft}^{2} \mathrm{hr}$ data compared to a prediction which assumes that equilibrium exists in the flow. Although the equilibrium wall temperature decreases more rapidly than the non-equilibrium wall temperature, the shape of the data is still much different than that predicted.

Groeneveld found that $\mathrm{Fr}-12$ decomposed at temperatures much above $600^{\circ} \mathrm{F}$ in the presence of moisture. Experience at the MIT Heat Transfer Laboratory indicates that temperatures as $10 \mathrm{w}$ as $300^{\circ} \mathrm{F}$ may cause Freon decomposition. Property values were found to change with time even at these low temperatures.

Wall temperatures in Cumo's experiments were as high as $570^{\circ} \mathrm{F}$. Thus, some Freon decomposition might have occured, leading to the odd wall temperature data. 


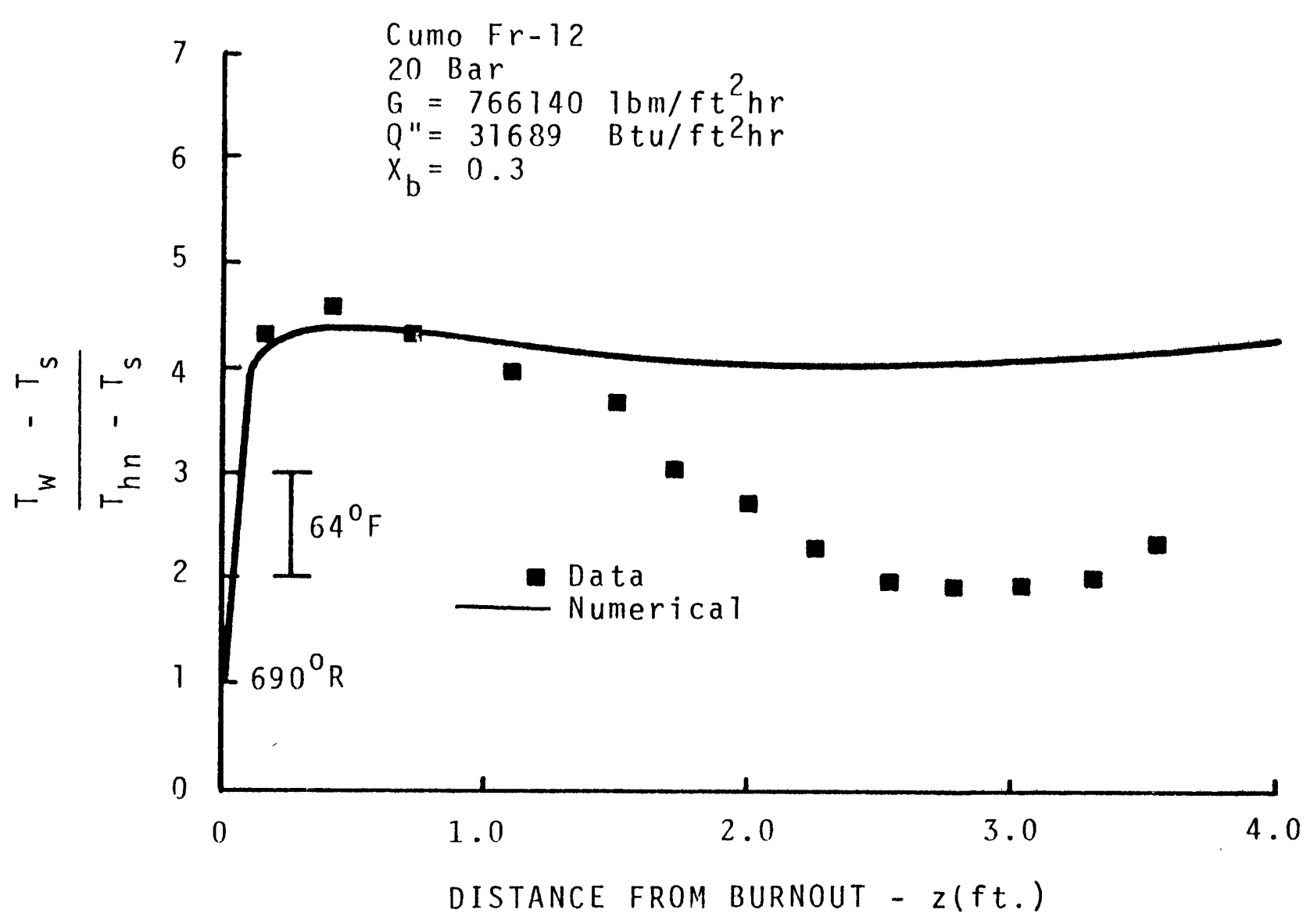

$\frac{1}{1}$

FIGURE 3-11 


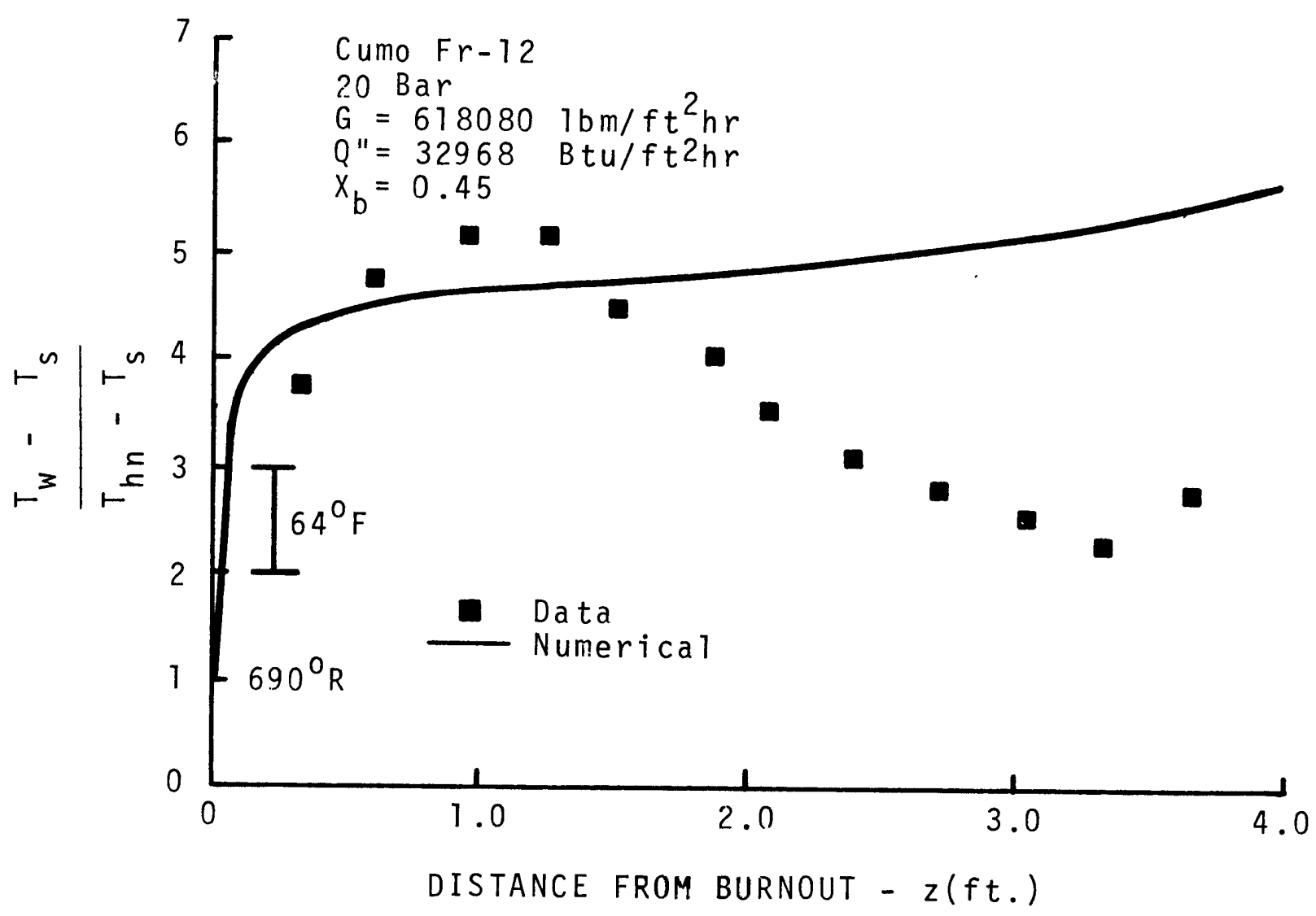

F IGURE 3-12 


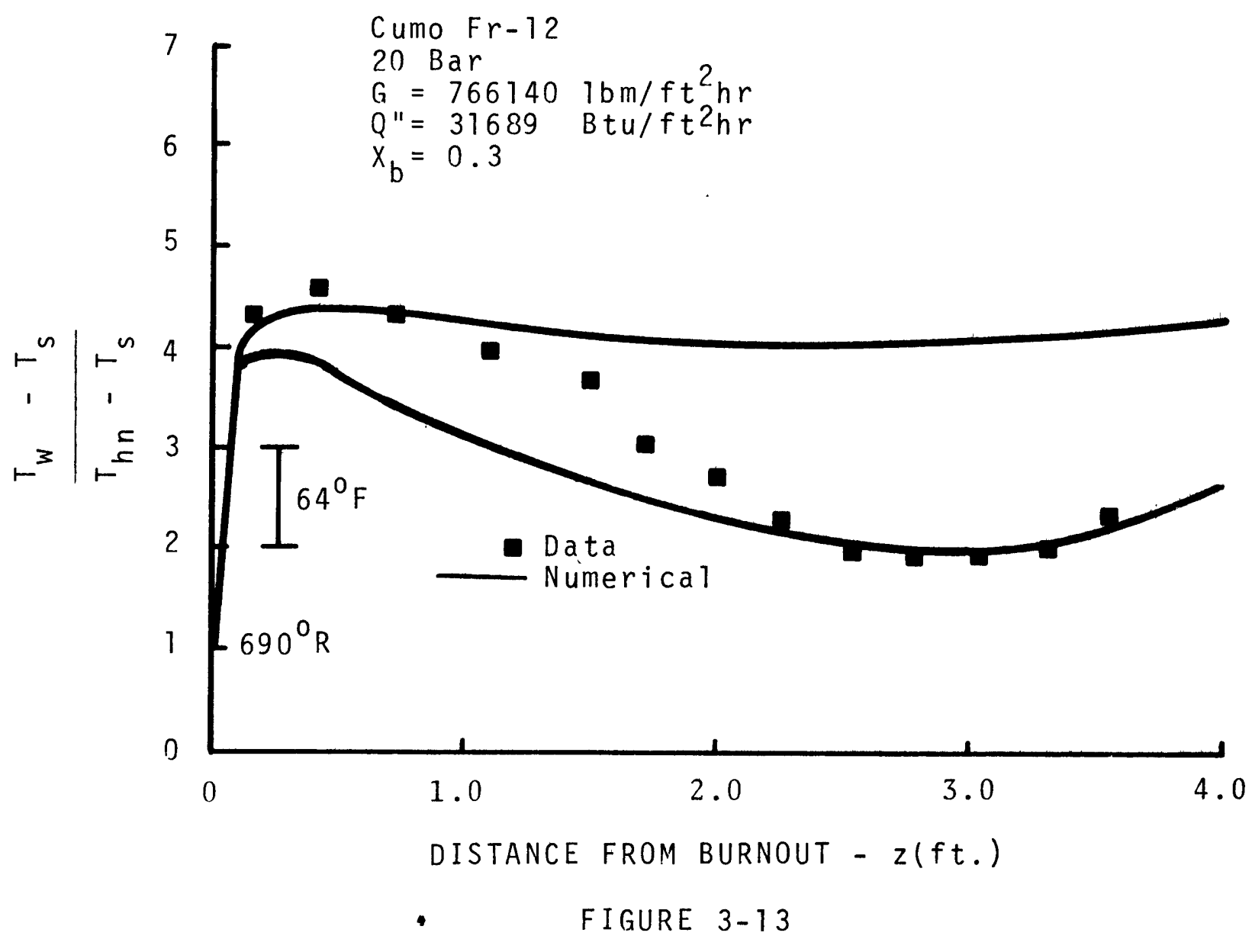




\section{2b Contribution of Individual Heat Transfer Mechanisms}

The importance of conduction and drop-wall interactions can be determined by looking at the heat transfer contribution from each.

Non equilibrium and the vapor heat transfer coefficient can be studied to determine qualitative effects on the wall temperature profile.

\section{Conduction}

Conduction had little effect on wall temperature profiles.Tube materials were either stainless steel or inconel in all of the experiments which were investigated in this study. The low thermal conductivity of these metals allows large temperature gradients to exist in the tube wall with little effect on the heat flux into the fluid.

Figure 3-14 shows wall temperature profiles with and without conduction present in the tube wall for nitrogen

$$
\left(G=130,000 \frac{1 \mathrm{bm}}{f t^{2} \mathrm{hr}}\right) \text {. At } z=0 \text {, the wall tempera- }
$$

ture is assumed to be the homogeneous nucleation temperature. Any heat conducted back into the wetted portion of the tube is absorbed by the liquid on the wall. The shape of the two curves in Figure 3-14 can be understood by examining the actual heat flux entering the fluid (the heat flux into 


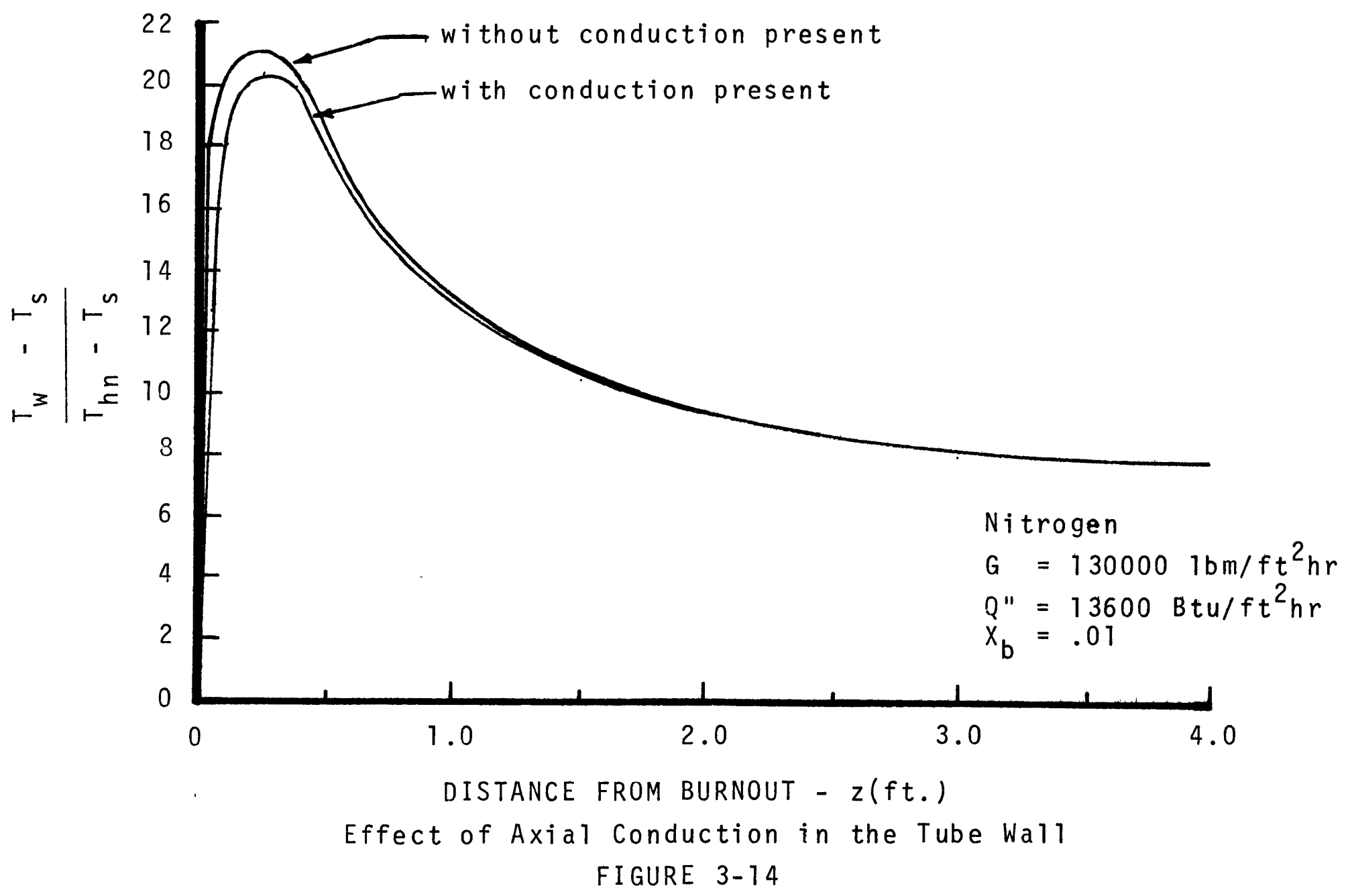


the tube minus the heat flux due to conduction). A plot of the local heat flux including conduction for the conditions in Figure 3-14 is shown in Figure 3-15.

At points before $z=.6 \mathrm{ft}$., the actual $\mathrm{flux}$ entering the fluid is always less than or equal to that predicted assuming no conduction in the tube wall. Thus, wall temperatures are lower in the conduction case than in the no conduction casela further explanation of equal fluxes follows). At points greater than $z=.6 \mathrm{ft}$., conduction increases the heat flux entering the fluid. Wall temperatures would be expected to be higher with conduction than with no conduction. However, another conduction related factor helps reduce wall temperatures.

All of the heat conducted out of the tube at the dryout point enters the wet wall region of flow. This heat is used to evaporate liquid wetting the wall. All liquid evaporated in this manner produces vapor at the saturation temperature. Thus, the quality at burnout is actually slightly higher than the burnout quality without conduction. More vapor is present at burnout, increasing the wal1-to-vapor heat transfer coefficient, and lowering wall temperatures at the beginning of the tube. Thus, the peak temperature at $z=.3 \mathrm{ft}$. is lower with conduction present than without conduction present. This effect also lasts sightly beyond the peak, lowering wall temperatures on down the tube. 


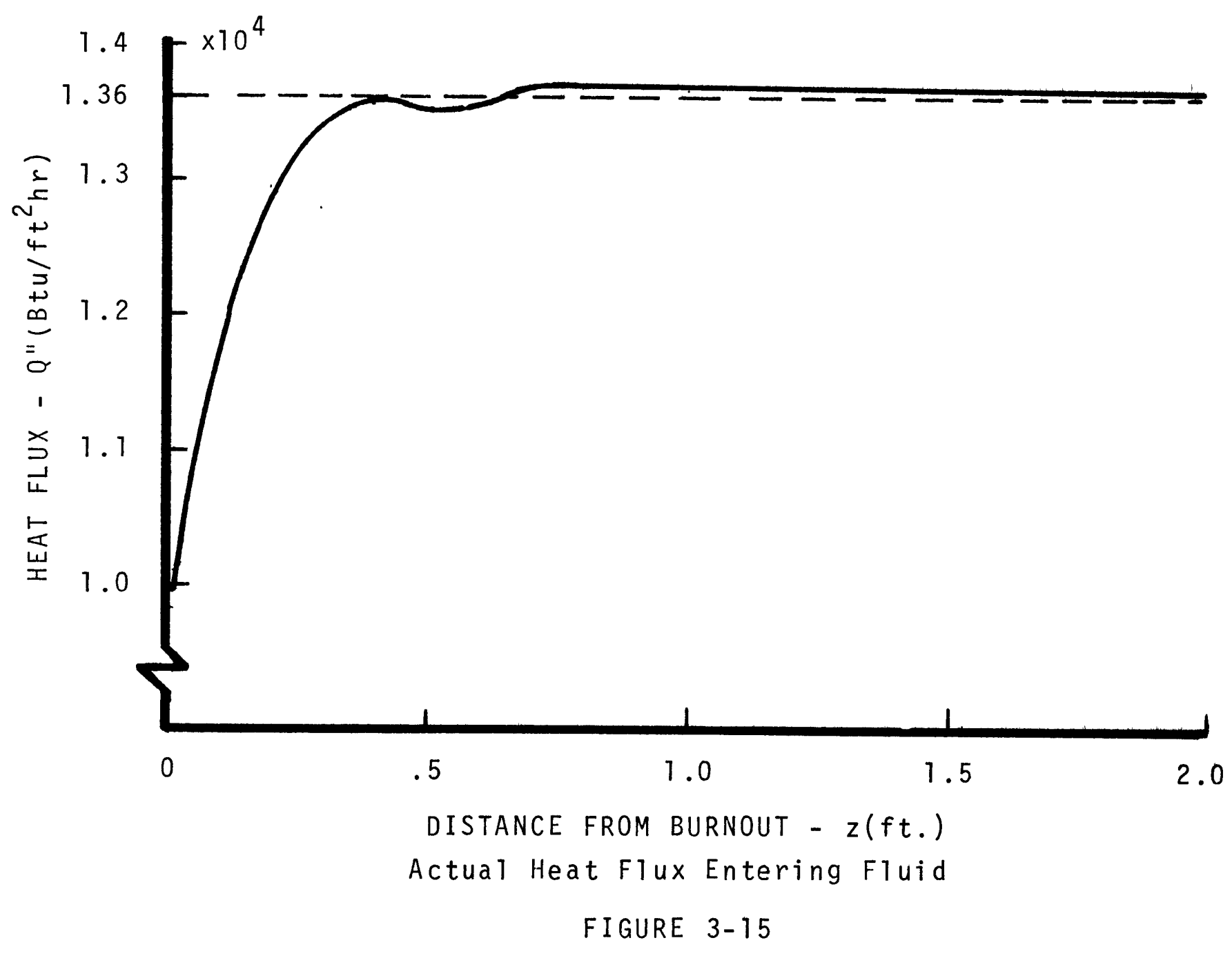




\section{Drop-Wall Interactions}

The effect of drop-wall interactions on the heat transfer analysis was slight. Equations (3-17) and (3-28) both contain a drop effectiveness term. Figure 3-16 shows the ratio of drop-wall heat transfer to the total heat transferred to the drops, calculated as the ratio of the last term to the total of Equation (3-17) or

$$
2 / 3 \frac{v_{p}}{v_{\ell}} \frac{D}{D_{T}} \varepsilon / \frac{d D}{d z}
$$

At burnout, no vapor superheat exists, and no heat is transferred to the drop from the vapor due to forced convection. Any drop evaporation is due to drop-wall interactions. As the vapor begins to superheat, the heat transfer from vapor to drop soon dominates. For all but only a short length immediately after burnout, the drop-wall heat transfer is less than $10 \%$ of the total heat transferred to the drops. The effect of drop-wall impacts on departure from equilibrium in the flow is therefore negligible.

Figure $3-17$ shows the ratio of the drop-wall contribution to the total heat flux from the wall, calculated as the ratio of the second term to the first term of the right side of Equation (3-28). The interplay between void 


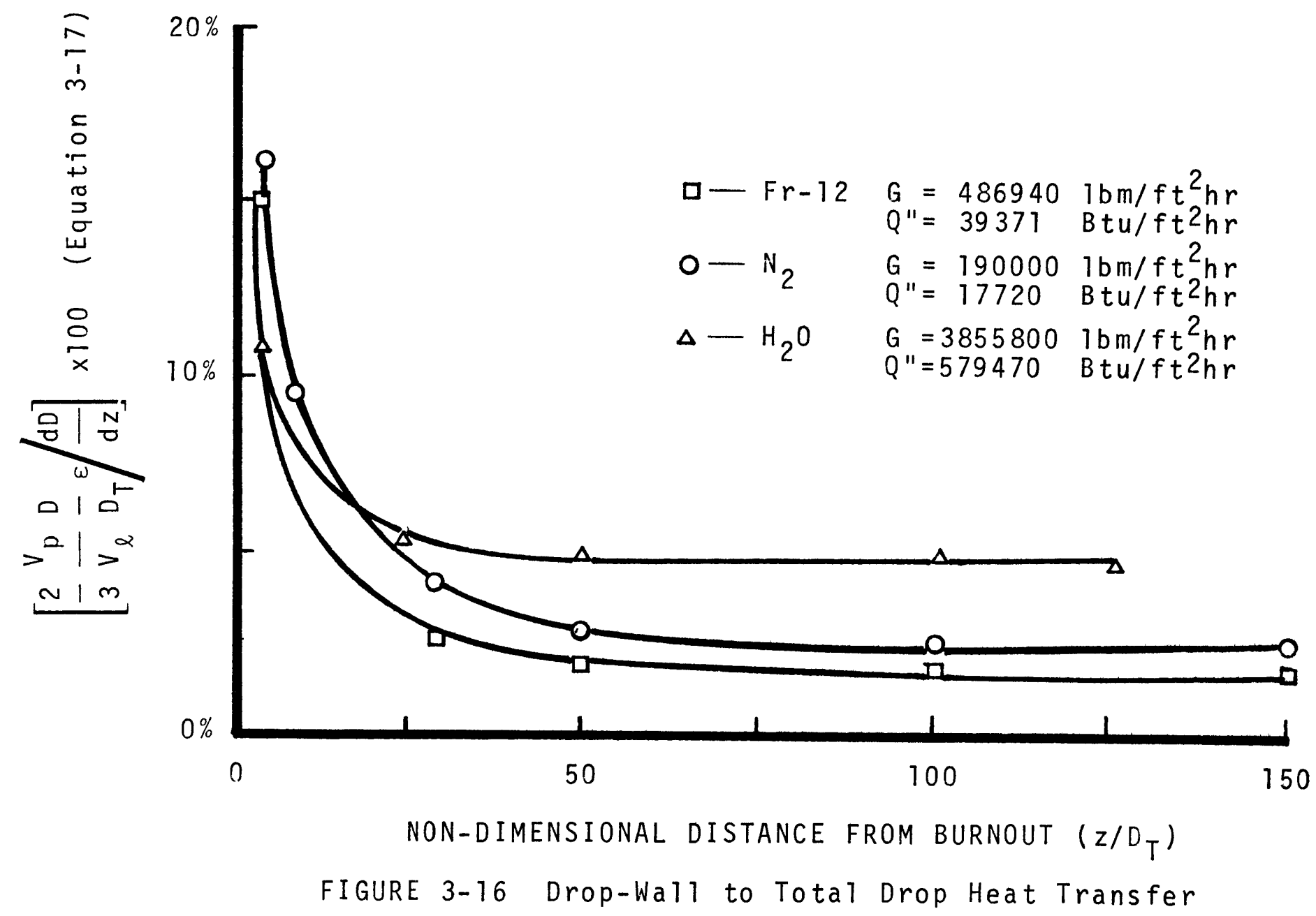




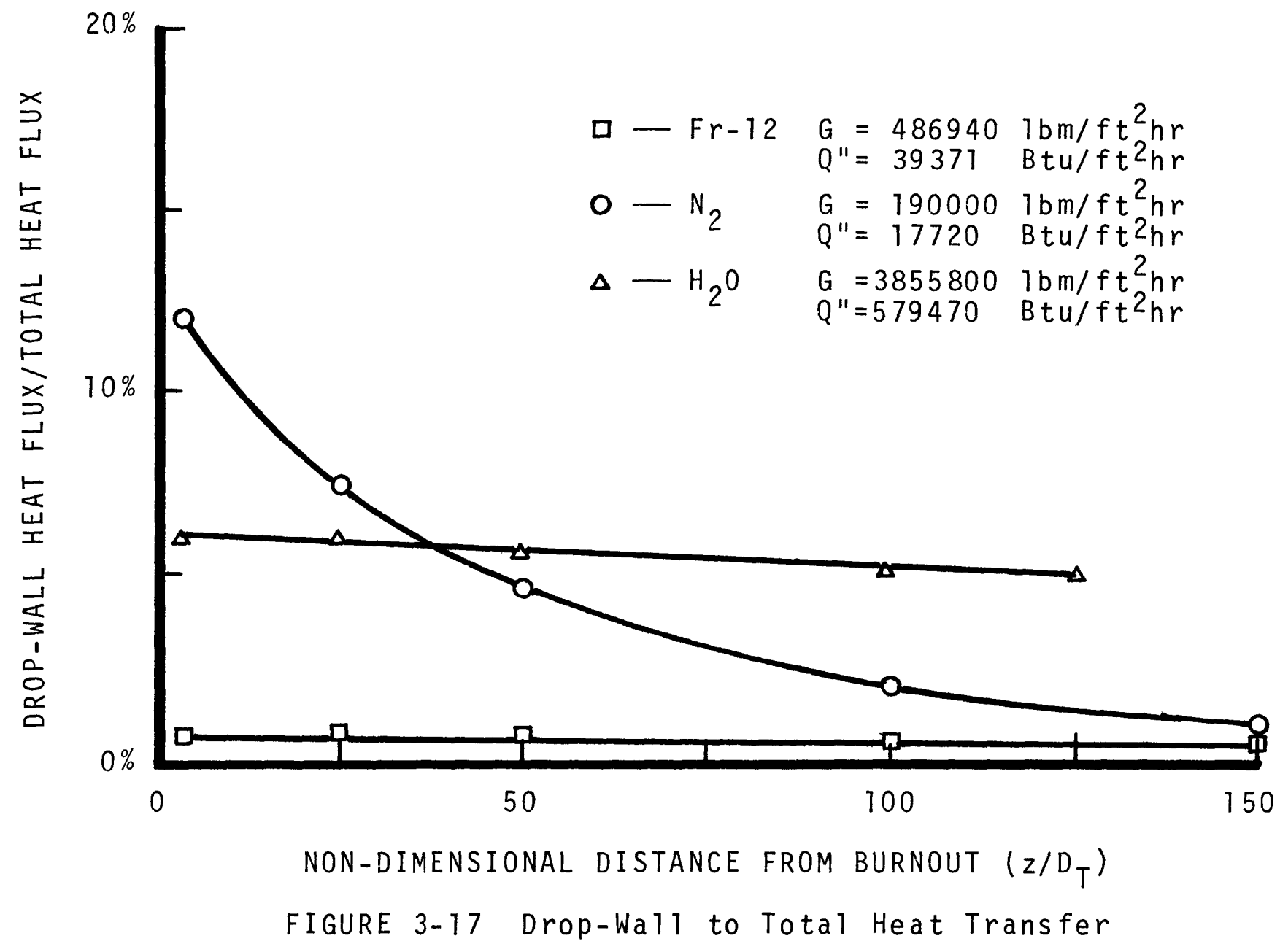


fraction, drop perpendicular velocity, and wall temperature determine the curve shapes in Figures $3-16$ and 3-17. Drop perpendicular velocity increases with distance from the burnout point because of increasing vapor velocity. This tends to increase the drop-wall heat transfer. Void fraction also increases due to decreasing drop diameter and slip ratio; this decreases the total drop-wall heat transfer. Wall temperature trends are dependent on fluid properties and flow conditions, and do not lend themselves to any general statements. However, in all cases investigated, the drop-wall heat transfer was small compared with the total flux at the wall.

Although no cases were found in which drop-wall interactions were important, some cases can be envisioned in which they could play an important role in determining wall temperatures. High drop perpendicular velocities in combination with low heat fluxes might produce such a situation. Heat transfer in swirling flows, or heat transfer from obstructions (i.e. reactor grid spacers) might be affected by drop-wall interactions.

3. Vapor to Drop Heat Transfer

The effect of changing the vapor-to-drop heat transfer coefficient by $10 \%$ or $20 \%$ from those calculated 
by Equation (3-33) results in very little change in predicted temperatures. However, if this heat transfer coefficient is infinite, the vapor remains at saturation temperature (the flow is equilibrium). The effect on wall temperature is shown for one example in Figure 3-13. This dramatic lowering of the wall temperature curve is caused by the increased wal1-to-vapor heat transfer coefficient resulting from the increased vapor velocity, as well the decreased vapor temperature.

4. Vapor Heat Transfer Coefficient

The magnitude of the vapor heat transfer coefficient alters the calculated wall temperature. Figure 3-10 shows wall temperatures calculated using two heat transfer coefficients. The higher the heat transfer coefficient, the lower the wall temperature.

In general, the degree of equilibrium in the flow determines the relative shape of the wall temperature curve, while the wall to vapor heat transfer coefficient determines the relative magnitude of the wall temperatures.

\subsection{Discussion of the Numerical Solution}

The dispersed flow model used in this study is similar to that used by Forsiund [ 3 ], Hynek [ 1 ], Groeneveld [5] 
and Plummer [18]. However, it differs in two important respects.

The new solution includes a consistent model for dropwall interactions and eliminates the need for variable constants in the analysis. Forslund's constant $k_{l} k_{2}$ and Groeneveld's drop wall separation distance were correlating factors used to fit dispersed flow data. $k_{1} k_{2}$ was found to be dependent on fluid type by Hynek, while Groeneveld's drop-wall separation distance was independent of wall temperature. Both seem intuitively incorrect. McCarthy's effectiveness model shows correct wall temperature trends, and agrees with single drop data. By accounting for the presence of the vapor temperature profile at the wall, her model can be applied to forced convective conditions.

Drop sizes in dispersed flow affect the degree of non equilibrium present. In this study, it was found that wall temperatures were affected very little by the initial choice of drop size in inverted annular burnout, as long as the drop diameter was larger than the diameter predicted using the free stream Weber number criterion, Equation (2-8). This was due to the rapid break up of drops down stream of the burnout point. Drop sizes quickly decreased to sizes dictated by the free stream Weber number criterion. Initial drop 
sizes in annular burnout do affect the degree of equilibrium in the flow. After burnout, they seldom break up due to the critical Weber number mechanism. Larger drop sizes therefore increase non-equilibrium in the flow because the liquid-vapor surface area, and therefore the vapor-drop heat transfer decreases with increasing drop size. Thus, increasing initial drop size in annular flow increases the predicted wall temperatures downstream.

Initial drop sizes predicted by previous analyses depended only on burnout conditions. In reality, droplets are all formed previously to dryout if the flow regime is annular, and drop sizes should depend on upstream conditions. The drop sizing model in this analysis includes this effect. The assumed linearmass distribution seems to be a reasonable approximation to what would be expected in heated flows. Wall temperatures predicted by this numerical model, using drop sizes calculated by this method, agree well with dispersed flow wall temperature data.

Extension of the model to regimes other than dispersed flow, as was done near burnout with Forslund's data, causes discrepancies in the predicted and actual wall temperatures. As the flow pattern becomes more like dispersed flow, the predicted and actual wall temperatures merge. 
The Dittus-Boelter and Heineman equations were used to predict wall-to-vapor heat transfer with reasonable success. However, as was suggested by Plummer, heat transfer equations developed for specific fluids might better predict data.

The numerical model is able to predict data for several fluids quite well, however, it requires computer implementation. As with all numerical solutions, it is difficult to predict intuitively the effect parameter perturbations have on the overall solution. In the next chapter, information gathered from the numerical solution will be used to simplify the governing equations, and a solution technique will be presented which needs no computer solution. 


\section{CHAPTER IV}

LOCAL CONDITIONS SOLUTION

\subsection{Formulations}

The analytic solution developed in Chapter III, (Equations $(3-4 a),(3-17),(3-20),(3-25),(3-28))$ requires a stepwise integration from burnout to predict wall temperatures downstream. By examining the results of these calculations for many different conditions, simplifying relationswere obtained which permit the prediction of wall temperatures at any position using known local conditions. The same basic equations are used in the local solution, but are modified including only the most important heat transfer effects.

Conduction in the tube wall has been shown to have little effect on predicted wall temperatures, and drop-wall heat transfer is important for only a very small distance down the tube from burnout. These two conclusions are the major reason why the local condition solution is possible. Before proceeding, several non-dimensional groupings can be identified which appear in many equations and help simplify calculations. They are presented here. 


$$
\begin{aligned}
& \tau=\frac{\rho_{v}{ }^{\sigma}{ }_{c}}{G^{2} D_{T}} \\
& \text { surface tension group 4-1 } \\
& A c=\frac{Q^{\prime \prime}}{G h_{f g}} \\
& G r=\frac{\rho_{v}\left(\rho_{\ell}-\rho_{v}\right) D_{T} g}{G^{2}} \quad \text { Gravity group }
\end{aligned}
$$

These originate from the droplet momentum equation and the definition of the Weber number.

\section{1 a Dryout Conditions}

Calculations necessary to determine initial drop diameters have been presented in Chapter II, and Section 3.1b. The numerical solution allows drops to break up after burnout due to a critical Weber number as they travel downstream. The governing equations can be greatly simplified if no further drop breakup occurs after burnout. In order to make this assumption, one of two conditions must hold.

1. No drop breakup actually occurs after burnout

or

2. An appropriate burnout drop diameter can be calculated which produces the same non-equilibrium in the flow as that which would occur if droplets 
present at burnout were allowed to break up as they travel downstream.

The remainder of this section is devoted to determining if condition 1 holds in the flow, and if it does not, what drop size should be used to cause condition 2 to hold.

Figure 4-1 shows the diameter history for three average drop sizes at burnout. Curve $C$ is the free stream Weber number criteria, Equation (2-8) for determining drop size. Drop size $D_{i}$ evaporates as it moves down the tube, and never intercepts the Weber number break-up curve, $C$. Thus, it correponds to condition 1 discussed above. Both drop sizes $D_{i j}$ and $D_{i j i}$ eventually intersect the critical Weber number curve, and would be expected to breakup. In order to determine when droplets break up, an analytic method for determining the equation for curve $C$ is needed.

$$
\text { Equation (2-8) defines this curve and can be written }
$$
in terms of the void fraction, $\alpha$ and slip ratio s

$$
\frac{D}{D_{T}}=\tau W_{c}-\frac{s^{2} \alpha^{2}}{(s-1)^{2}} \frac{1}{x^{2}}
$$

The droplet momentum Equation (3-4) 


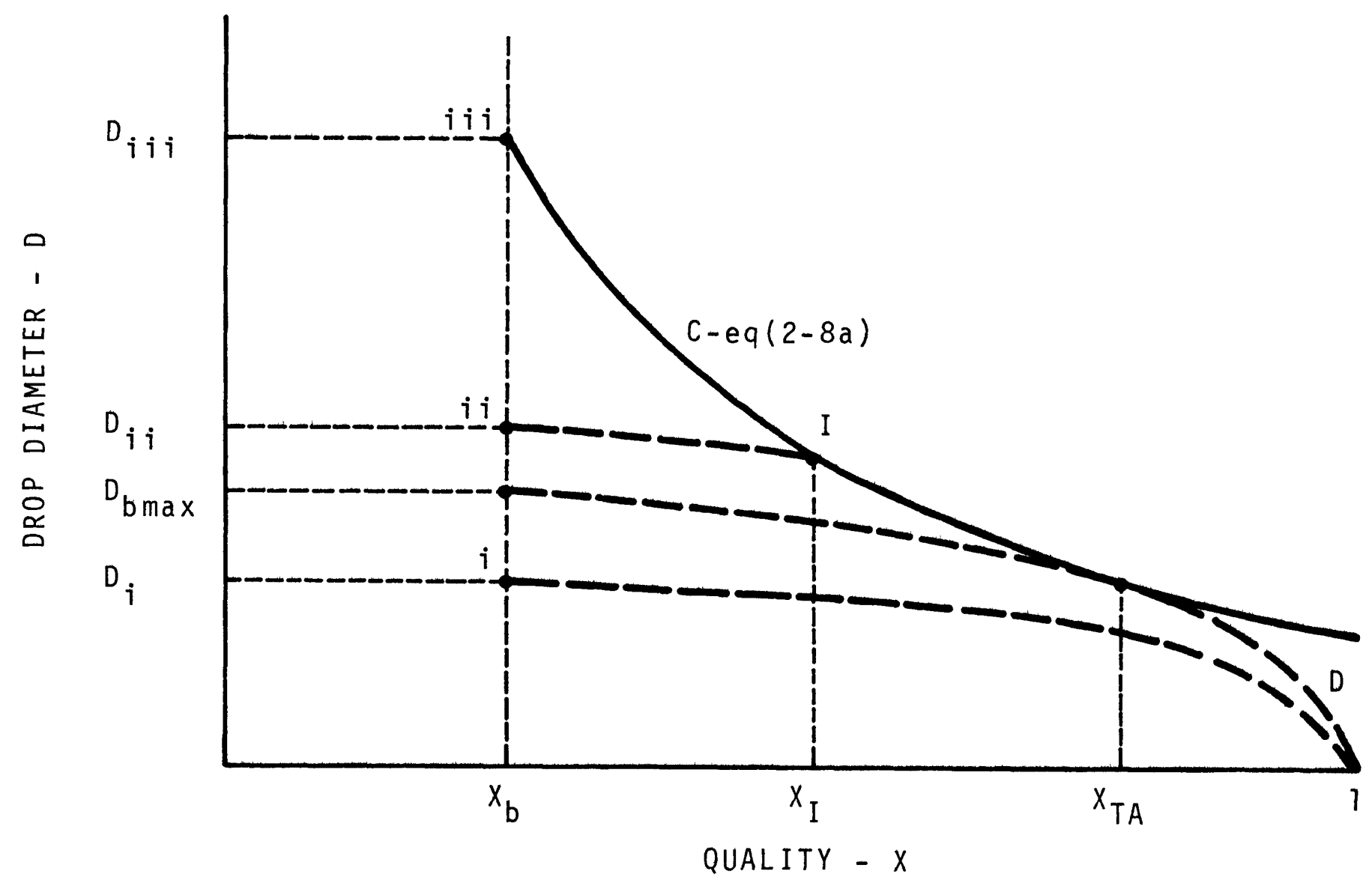

$\frac{1}{0}$

FIGURE 4-1 Drop Diameter History after Burnout 


$$
\rho_{\ell} v_{\ell} \frac{d V_{\ell}}{d z}=-g\left(\rho_{\ell}-\rho_{v}\right)+\frac{3}{4} C_{D} \rho_{v} v_{\ell}^{2}(s-1)^{2} \frac{1}{D} \quad 3-4
$$

can be simplified by assuming that the change in actual quality with distance is approximately equal to the change in equilibrium quality with distance. This assumption is discussed in Appendix A-1, and is shown to be a good approximation. Also, the assumption that $x \frac{d \frac{1}{\delta \alpha}}{d z} \ll \frac{1}{\delta \alpha} \frac{d x}{d z}$, used in Section 3.1 a should be valid at any point in the flow using the same argument as presented in Section 3.1a. Using the definition of liquid velocity, Equations (2-5) and (2-6), the momentum equation can be written as

$$
\frac{16}{3} \frac{\rho_{\ell}}{\rho_{V}} \frac{A C}{C_{D}} \frac{D_{0}}{D_{T}}=-\frac{4}{3} G r \frac{s^{2} \alpha^{2}}{C_{D} X} \frac{D_{0}}{D_{T}}+(s-1)^{2} X
$$

Equation (3-4b) can be solved for (s - 1) and inserted into Equation $(2-8 a)$. Solving for $\frac{D}{D_{T}}$ yields,

$$
\frac{D}{D_{T}}=\left[3 \tau W_{e_{C}} C_{D} s^{2} \alpha^{2} /\left(16 A c \frac{\rho_{\ell}}{\rho_{V}} x+4 G r s^{2} \alpha^{2}\right)\right]^{1 / 2}
$$

For many cases in dispersed flow, the value of so is near 1 , and an explicit equation for $D$ can be obtained. 


$$
\left.\frac{D}{D_{T}}=\left[\begin{array}{llll}
3 & W_{C}{ }_{C} C_{D} /(16 A c & \frac{\rho_{\ell}}{\rho_{v}} x+4 & G r
\end{array}\right)\right]^{1 / 2}
$$

This is an equation for curve $C$ in Figure 4-1. Thus, the drop diameter determined by the critical Weber number criterion can be determined at any quality once property values, $C_{D}$, and the flow conditions are known. Reynolds numbers are normally high enough that $C_{D}=0.4$ may be used. An equation for free stream slip at any point in the flow can be obtained by solving Equation 3-4b for $S$.

$$
\begin{gathered}
S=\left[1+\left\{\left(1-\frac{4}{3} \operatorname{Gr} \frac{\alpha^{2}}{x^{2}} \frac{1}{C_{D}} \frac{D}{D_{T}}\right)\left(\frac{16}{3} \frac{\rho_{\ell}}{\rho_{V}} A C \frac{1}{x C_{D}} \frac{D}{D_{T}}-1\right)+1\right\}^{1 / 2}\right] / \\
{\left[1-\frac{4}{3} G r \frac{\alpha^{2}}{x^{2}} \frac{1}{C_{D}} \frac{D}{D_{T}}\right]}
\end{gathered}
$$

In many cases, this equation can also be simplified. If

$$
\frac{4}{3} \operatorname{Gr} \frac{\alpha^{2}}{x^{2}} \frac{1}{C_{D}} \frac{D}{D_{T}} \ll 1
$$

which is generally true except at lower quality, then

$$
s-i=\left(\frac{16}{3} \frac{\rho_{\ell}}{\rho_{V}} A C \frac{1}{x C_{D}} \frac{D}{D_{T}}\right)^{1 / 2}
$$


Reynolds numbers are normally high enough so that $C_{D}$ is approximately .4, and Equation (4-7) can be used to define the slip for drop diameter, $D$ at any actual quality, $X$. Returning to Figure 4-1, the three drop sizes $D_{i j i}$, $D_{i j}$, and $D_{i}$ can be investigated separately.

1. Inverted Annular Flow - Diii

Burnout drop sizes may be characterized by the free stream Weber number, $D_{i j i}$, particularly in inverted annular flow. Beyond burnout, the drop diameter actually follows curve $C$ stepwise until point $X_{T A}$ where evaporation dominates, and the drop diameter follows curve $D$, Equation (2-14).

An average drop diameter which partially accounts for droplet breakup after burnout can be calculated by integrating Equation (4-5) from $x=x_{b}$ to $x=x_{T A}$, assuming that $D_{T A}$ (Figure 4-1) characterizes the flow from $x=x_{T A}$ to $X=1$, and that because breakup is so rapid, evaporation has little effect in determining drop diameter until $X=X_{T A}$.

$$
\frac{D_{0}}{D_{T}}=\frac{1}{1-x_{b}}\left[\int_{X_{b}}^{X_{T A}} \frac{D}{D_{T}} d x+\frac{D_{T A}}{D_{T}}\left(1-x_{T A}\right)\right] \quad 4-8
$$


Inserting Equation (4-5) for $\frac{D}{D_{T}}$ and integrating, as suming constant properties,

$$
\begin{aligned}
\frac{D_{0}}{D_{T}}= & \frac{1}{1-X_{b}}\left[\frac { 3 \sqrt { \tau W _ { C } } C _ { D } } { A _ { C } } \frac { \rho _ { v } } { \rho _ { \ell } } \left\{\left(\frac{16}{3} A c \frac{\rho_{\ell}}{\rho_{V}} \frac{X_{T A}}{C_{D}}+\frac{4}{3} \frac{G r}{C_{D}}\right)^{1 / 2}-\right.\right. \\
& \left.\left.\left(\frac{16}{3} A C \frac{\rho_{\ell}}{\rho_{v}} \frac{X_{b}}{C_{D}}+\frac{4}{3} \frac{G r}{C_{D}}\right),{ }^{1 / 2}\right\}+\left(1-X_{T A}\right) \frac{D_{T A}}{D_{T}}\right]
\end{aligned}
$$

$X_{T A}$ can be calculated by equating the slopes and magnitudes of the evaporation curve, Equation (2-14) and the free stream Weber number criterion, Equation (4-5) at $X_{T A} \cdot$

$$
x_{T A}=\frac{3}{5}-\frac{1}{10} \frac{G r}{A c} \frac{\rho_{v}}{\rho_{\ell}}
$$

$D_{T A}$ can be calculated using Equation $(4-5)$ at $X_{T A}$

$$
\frac{D_{T A}}{D_{T}}=\left[3 \tau W_{C} C_{D} /\left(16 A c \frac{\rho_{\ell}}{\rho_{v}} X_{T A}+4 G r\right)\right]^{1 / 2} 4-11
$$

Equations $(4-10)$ and $(4-11)$ define the tangent point of the critical Weber number criterion and the evaporation curve for any flow condition. 


\section{Annular Flow $-D_{i j}$ and $D_{i}$}

The techniques in Chapter II allow calculation of the average drop size actually present at burnout, $\bar{D}$, Equation (2-18). With the previous assumptions, this diameter can now be calculated. Equations (2-18) and (2-19) can be used to determine $\bar{D}$ once values for $D_{b}$ and the free stream slip, $s$ are known. The critical Weber number drop diameter at burnout can be evaluated using Equation (4-5) at the burnout quality, $x_{b}$.

$$
\frac{D_{b}}{D_{T}}=\left[3 \tau W_{C} \quad C_{D} /\left(16 A c \frac{\rho_{\ell}}{\rho_{v}} X_{b}+4 G r\right)\right]^{1 / 2} 4-12
$$

The free stream slip for drop diameter $D_{b}$ at burnout is given by Equations (4-6) or (4-7), evaluated at $x=x_{b}$ with $D=D_{b}$.

$$
s-1=\left(\frac{16}{3} \frac{\rho_{l}}{\rho v} A c \frac{1}{X_{b} C_{D}} \frac{D_{b}}{D_{T}}\right)^{1 / 2}
$$

Equation (4-12) assumes that $s \alpha \approx 1$. The value of s $\alpha$ should be highest using the free stream drop diameter at burnout, since dropdiameters decrease in size as the drops move downstream. If the value of $s \alpha$ calculated using information from Equations (4-12) and (4-7a) or (4-6) is 
larger than approximately $1.7, D_{b}$ should be calculated using Equation (4-4) evaluated at $x_{b}$. No cases in this investigation required solving Equation (4-4) iteratively to determine $D_{b}$, however, high $s \alpha$ values could occur in some flow situations.

A criterion must now be established to determine if $\bar{D}$ will break up beyond the burnout point as it travels down the tube.

The value of $D_{T A}$, Equation (4-11) can be used to determine the maximum drop diameter at burnout which would not break up as ittravels along the tube, Dbmax. Figure 4-1. Using the same reasoning as was used in arriving at Equation (2-14) with no droplet breakup,

$$
\frac{D_{b \max }}{D_{T A}}=\left[\frac{1-x_{b}}{1-x_{T A}}\right]^{1 / 3}
$$

3. Annular Flow - Dij

If $D_{b \max }$ is less than $\bar{D}$, Equation (2-18), (i.e. $D_{i j}$, Figure 4-1), the drops will break up as they travel down the tube. A procedure similar to that used for $D_{i i i}$ can be used to determine an approximate drop size for this case.

The point $X_{I}$ where drops with size $D_{i j}$ at burn- 
out intercept curve $C$, the Weber number curve, can be approximated by equating $D_{i j}$ (or $\bar{D}$ ) to $D$ in Equation $(4-5)$.

$$
X_{I}=\frac{3}{16} \frac{C_{D}}{A c} \frac{\rho_{V}}{\rho_{l}}\left[\left(\frac{D_{T}}{\bar{D}}\right)^{2} \tau W_{c}-\frac{4}{3} \frac{G r}{C_{D}}\right]
$$

From point $X_{I}$ to $X_{T A}$, the same integration can be performed as in the inverted annular case (1). The average drop diameter becomes

$$
\begin{aligned}
& \frac{D_{0}}{D_{T}}=\frac{1}{1-x_{b}}\left[\frac{\bar{D}}{D_{T}}\left(x_{I}-x_{b}\right)+\frac{D_{T A}}{D_{T}}\left(1-x_{T A}\right)+\frac{3}{8} \frac{\sqrt{\tau W e} C_{D}}{A C} \frac{\rho_{V}}{\rho_{\ell}} \bullet\right. \\
& \left.\left\{\left(\frac{16}{3} A c \frac{\rho_{\ell}}{\rho_{v}} \frac{X_{T A}}{C_{D}}+\frac{4}{3} \frac{G r}{C_{D}}\right)^{7 / 2}-\left(\frac{16}{3} A c \frac{\rho_{\ell}}{\rho_{v}} \frac{X_{I}}{C_{D}}+\frac{4}{3} \frac{G r}{C_{D}}\right)^{1 / 2}\right\}\right] 4-15
\end{aligned}
$$

\section{Annular Flow $-D_{i}$}

Fortunately, the most commonty occuring case in annular flow is $\bar{D}<D_{b \max }$, and the appropriate burnout drop diameter is just $D_{0}=\bar{D}$, Equation (2-18).

Free stream slip at burnout can be calculated using Equations (4-6) or (4-7). Void fraction is thengiven by Equation (2-7), and vapor and liquid velocities by $(2-5)$ and (2-6) respectively. 
4.1b Governing Equations

An analytic solution must be able to predict local conditions based on known parameters. In this solution scheme, the following parameters are assumed known.

Initial Conditions

$\begin{array}{lll}D_{0} & - & \text { drop diameter } \\ x_{b} & - & \text { burnout quality }\end{array}$

Flow Conditions

$\begin{array}{lll}G & - & \text { mass flux } \\ Q^{\prime \prime} & - & \text { heat flux } \\ D_{T} & - & \text { tube diameter } \\ \text { Fluid Properties } & & \end{array}$

The parameters to be determined by the analysis are,

Local Conditions

\begin{tabular}{|c|c|c|}
\hline$x$ & - & actual quality \\
\hline$T_{v}$ & - & vapor temperature \\
\hline$v_{v}$ & - & vapor velocity \\
\hline$T_{w}$ & - & wall temperature \\
\hline
\end{tabular}


Once drop diameter $D_{0}$ is determined using the analysis in section 4-1a, the governing equations presented in section 3-1b must be used to determine local conditions downstream of burnout. To do this, the conclusions from the step-wise analytical model are used to develop the simplified equations for the local conditions model.

The drop diameter gradient, Equation (3-17) can be simplified by assuming that the drop-wall interaction is negligible as suggested in the conclusions from the numerical model.

$$
\frac{d D}{d z}=-2 \frac{h_{D}\left(T_{v}-T_{s}\right)}{v_{\ell} \rho_{\ell} h_{f g}} \quad 4-16
$$

In section 4-1a, we have determined an initial drop diameter, $D_{0}$ which can be used assuming no droplet breakup occurs downstream of burnout. The quality gradient, Equation (3-20)

$$
\frac{d x}{d z}=-\frac{3(1-X)}{D} \frac{d D}{d z}
$$

can be integrated from $x_{b}$ to $x$. 


$$
\frac{D}{D_{0}}=\left[\frac{1-x}{1-x_{b}}\right]^{1 / 3}
$$

and $D$ from Equation (4-17) can be substituted back into Equation (3- 20)

$$
\frac{d X}{d z}=-3 \frac{(1-X)}{D_{0}}\left[\frac{1-X_{b}}{1-X}\right]^{1 / 3} \frac{d D}{d z}
$$

The energy balance on the vapor, Equation (3-25) can be integrated assuming $C_{p}$ remains constant, which is a good approximation for all of the data analyzed. The vapor energy balance then becomes,

$$
T_{v}-T_{s}=\frac{4 Q "}{G X C_{p} D_{T}} z-\frac{h_{f g}}{C_{p}}\left[\frac{X-X_{b}}{X}\right]
$$

Equation (4-16) can be substituted into Equation (4-18) for $\frac{d D}{d z}$, and Equation (4-19) can be substituted into the resulting Equation for $\left(T_{v}-T_{s}\right)$.

$$
\frac{d x}{d z}=6 \frac{(1-x)}{x}\left[\frac{1-x_{b}}{1-x}\right]^{1 / 3} \frac{h_{D}}{V_{\ell} \rho_{\ell} D_{0} h_{f g}}\left[\frac{4 Q^{\prime \prime}}{G C_{p}} \frac{z}{D_{T}}-\frac{h_{f g}}{C_{p}}\left(x-x_{b}\right)\right]
$$


using an energy balance, the equilibrium quality is

$$
x_{e q}-X_{b}=\frac{4 Q^{\prime \prime}}{G h_{f g}} \frac{D_{T}}{D_{T}}
$$

and

$$
N u_{D}=\frac{h_{D} D}{k_{v}}
$$

Then defining

$$
\operatorname{Re}_{D}{ }^{\prime}=\frac{\rho_{v} v_{\ell} D}{\mu_{v}}
$$

Equation (4-20) becomes

$$
\begin{gathered}
\frac{2}{3} \frac{D_{0}}{D_{T}} \frac{1}{\left(1-X_{b}\right)} 1 / 3 \frac{Q "}{G h_{f g}} \operatorname{Pr}_{v} \frac{\rho_{\ell}}{\rho_{v}} \frac{R_{D}{ }^{\prime}}{N u_{D}} \frac{X}{(1-x)^{2 / 3}} \frac{d x}{d x_{e q}} \\
=\left(x_{e q}-x\right)
\end{gathered}
$$

This equation is now a function of property values, flow, conditions, quality, equilibrium quality, and $\underset{\operatorname{Re}_{D}}{r a t i o}, \frac{\operatorname{Re}_{D}}{N u_{D}}$. With the functional dependence of the ratio $\frac{\mathrm{Re}_{D}}{\mathrm{Nu}}$ on $X$, and with the assumption that property values remain approximately constant, this is a differential equation in terms of $x$ and $x_{e q}$.

An equation for liquid velocity results from using 
Equations $(2-5)$ and $(2-6)$

$$
V_{\ell}=\frac{G X}{\rho_{V} s \alpha}
$$

Equation (4-24) can be substituted into Equation (4-22) for $V_{\ell}$ and Equation (4-17) into (4-22) for $D$. Then

$$
\operatorname{Re}_{D}{ }^{\prime}=\frac{G X}{s \alpha \mu_{v}} D_{0}\left[\frac{1-X}{1-X_{b}}\right]^{1 / 3}
$$

If the variation of the product $s \alpha$ is small, as assumed in section $4-1 a$,

$$
\operatorname{Re}_{D}^{\prime} \propto x(1-x)^{1 / 3}
$$

The drop Nusselt number, $N u_{D}$ is given by Equations $(3-31)$ and (3-32)

$$
N u_{D}=\left(2+.6\left[\frac{\rho_{f}\left(v_{v}-v_{\ell}\right) D}{\mu_{f}}\right]^{1 / 2} P r_{f}^{1 / 3}\right)(1+B)^{-1}
$$

where

$$
1+B=1+\frac{C_{p}\left(T_{v}-T_{s}\right)}{h_{f g}}
$$

However, the definition of equilibrium quality, Equation 
(4-21) can be substituted into the energy equation (4-19) for $\frac{4 Q^{\prime \prime} z}{G D_{T}}$ to give the quantity $\frac{C_{p}}{h_{f g}}\left(T_{v}-T_{S}\right)$ in terms of quality and equilibrium quality.

$$
\frac{C_{p}}{h_{f g}}\left(T_{v}-T_{s}\right)=\frac{X_{e q}}{x}-1
$$

or

$$
1+B=\frac{x_{e q}}{x}
$$

The Ranz-Marshall portion of $N u_{D}$, Equation (3-32) can be investigated separately

$$
N u_{0}=2+.6\left[\frac{\rho_{f}\left(v_{v}-v_{\ell}\right) D}{\mu_{f}}\right]^{1 / 2} P r_{f}{ }^{1 / 3} \quad 4-29
$$

The second term dominates over the major portion of the quality range, and

$$
N u_{0} \propto\left[\frac{\rho_{f}\left(v_{v}-v_{l}\right) D}{\mu_{f}}\right]^{1 / 2}
$$

$v_{v}-v_{\ell}$ can be written in terms of slip and liquid velocity, 


$$
N u_{0} \propto\left[\frac{G \times(s-1) D}{s \alpha \mu_{f}}\right]^{\frac{1}{2}}
$$

Equation (4-7) indicates that

$$
s-1 \propto\left(\frac{D}{x}\right)^{1 / 2}
$$

substituting Equation (4-32) into (4-31) and using Equation $(4-17)$ to replace $D$

$$
N u_{0} \propto\left[\frac{G}{s \alpha \mu_{f}}\right]^{1 / 2}\left[x^{1 / 2} D_{0}^{3 / 2}\left[\frac{1-x}{1-x_{b}}\right]^{1 / 2}\right]^{1 / 2} 4-33
$$

if $s \alpha$ is again assumed to remain approximately constant, Equations $(4-33)$ and $(4-28)$ can be combined to give the Nusselt number's dependence on quality.

$$
N u_{D} \propto x^{1 / 4}(1-x)^{1 / 4} \frac{x}{x_{e q}}
$$

From Equations (4-34) and (4-26), it can be seen that

$$
\frac{\operatorname{Re}_{D}{ }^{\prime}}{X(1-x)^{1 / 3}} \frac{x^{1 / 4}(1-x)^{1 / 4}}{N u_{D}} \frac{x}{x_{e q}} \cong \text { Constant }
$$

Equation (4-23) can now be multiplied and divided by 
$\frac{x^{1 / 4}}{(1-x)^{1 / 12} x_{e q}}$ to eliminate the dependence of $\left[\frac{R e_{D}}{N u_{D}}\right]$
on quality.

$\frac{2}{3} \frac{D_{0}}{D_{T}} \frac{1}{\left(1-x_{b}\right)^{1 / 3}} \frac{Q^{\prime \prime}}{G h_{f g}} \operatorname{Pr}_{v_{\rho_{v}}} \frac{\rho_{\ell}}{N u_{0}}\left[\frac{R e_{D}}{x^{3 / 4}(1-x)^{1 / 2}}\right]$

$$
\frac{x^{3 / 4} x_{e q}}{(1-x)^{7 / 12}} \frac{d x}{d x_{e q}}=\left(x_{e q}-x\right)
$$

The quantity

$K=\frac{2}{3} \frac{D_{0}}{D_{T}} \frac{1}{\left(1-X_{b}\right)} 1 / 3 \frac{Q^{\prime \prime}}{G h_{f g}} \operatorname{Pr}_{v_{v_{V}}} \frac{\rho_{\ell}}{\rho_{V}}\left[\frac{\operatorname{Re}_{D}^{\prime}}{N_{0}} \frac{1}{X^{3 / 4}(1-x)} 1 / 12\right] \quad 4-37$

should now remain constant with quality.

Figure 4-2 shows the variation of $K$ with quality as calculated by the numerical solution scheme described in Chapter III. For a 11 of the cases shown, except $\mathrm{N}_{2}$, no drop breakup occurs, and $K$ remains relatively constant, justifying the previous assumptions. The data shown for $\mathrm{N}_{2}(\nabla)$ is for an inverted annular burnout. The drops break up from $D_{i j i}$ along curve $C$ of Figure 4-1 up to a quality of about $60 \%$. Therefore, the actual magnitude of $\mathrm{K}$ for $\mathrm{H}_{2}$ shown in Figure 4-2, decreases from a high 


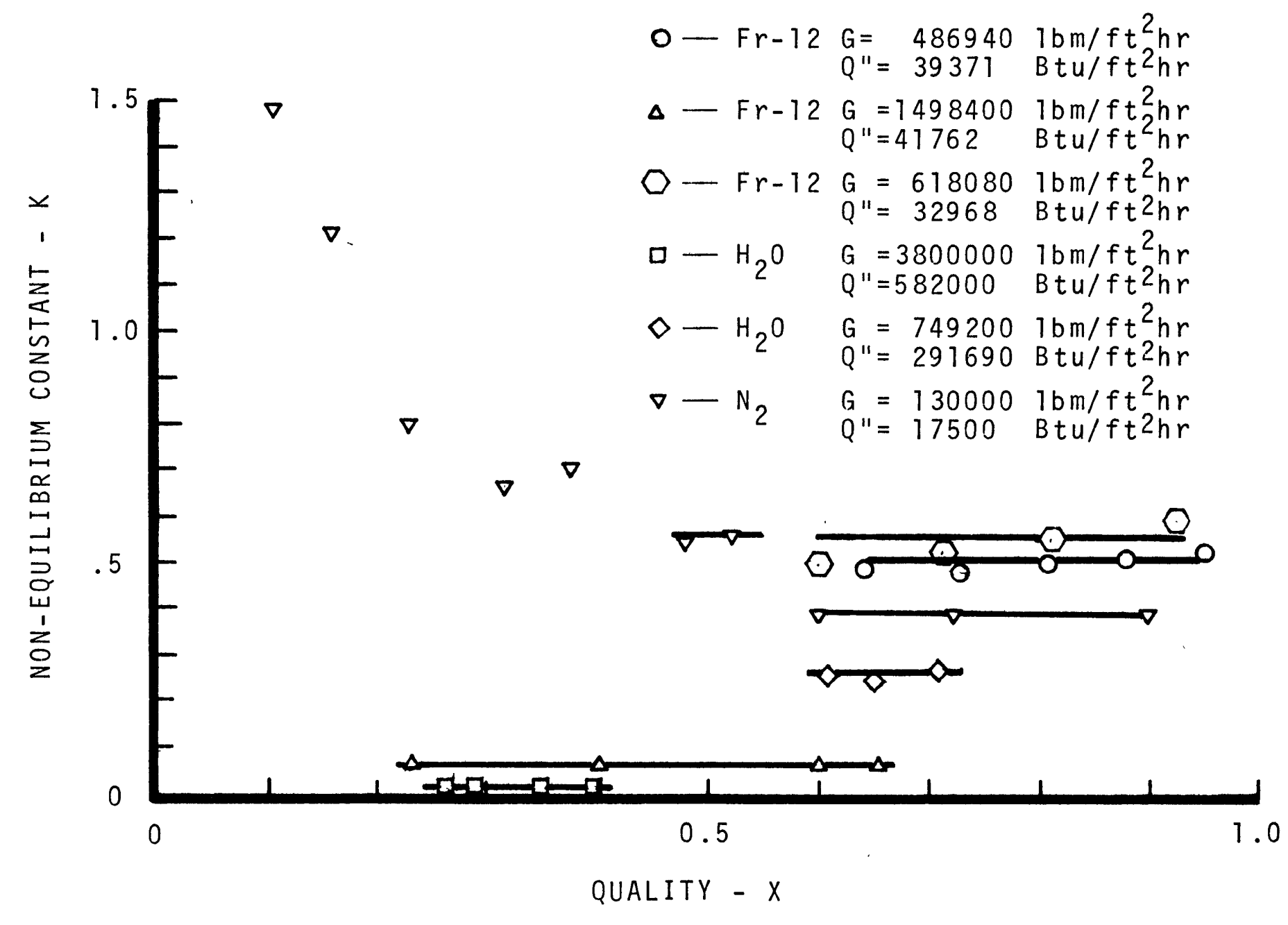

FIGURE 4-2 Non-Equilibrium Constant 
value and becomes essentially constant beyond $X=60 \%$. In this case, an average value of $K$ would be predicted as 0.59 using the integrated average drop diameter from Equation (4-9). Therefore, $K$ can always be determined from Equation (4-37) using only burnout conditions. Also for this case, $X_{T A}$ predicted from Equation (4-10) is $60 \%$, beyond which $K$ is constant in Figure 4-2.

Even though $K$ varies signifigantly for this case, the predicted wall temperatures shown later in Figure 4-13 agree well with this data.

Equation (4-36) can be rewritten in terms of $K$, $x$, and $x_{e q}$.

$$
K \frac{x^{3 / 4} x_{e q}}{(1-x)^{7 / 12}} \frac{d x}{d x_{e q}}=\left(x_{e q}-x\right) \quad 4-38
$$

This equation can now be integrated for various values of $K$ and burnout quality. Graphs of $x$ vs. $\left(x_{e q}-x_{b}\right)$ as a function of $K$ for burnout qualities from .1 to .9 are shown in Figures 4-3 through 4-11. For any burnout quality, and value of $K$, the actual quality can be determined knowing the equilibrium quality at the point of interest.

As can be seen from the figures, as the value of $k$ increases, the non-equilibrium in the flow increases. A 
value of $K=0$ indicates that equilibrium exists in the flow. This corresponds to a drop Nusselt number of $\infty$ in Equation (4-37), which would mean that all heat goes into evaporating the drops.

The slopes of the $k$ curves must be zero at the burnout quality as indicated by Equation (4-36) when $x_{e q}=x_{b}$ $=X$. Because the vapor temperature is saturation temperature at burnout, no drop evaporation can occur initialiy, and the quality must remain at $x_{b}$ until enough vapor superheat is available to begin to evaporate drops. This effect is most evident in the high burnout quality $K$ curves (Figures 4-10 and 4-11) since very little liquid is present at burnout. In the lower burnout quaility $K$ curves, this effect is much less pronounced since much more liquid is present in the flow. As $K$ increases, the effect also becomes more prominent as the vapor to liquid heat transfer mechanism is becoming less effective.

At a quality of 1 , the slopes of the $K$ curves must also be zero as indicated by Equation (4-36). At a quality near one, very little liquid is available to evaporate in the flow, and the slope of the constant $K$ curve must decrease to zero as the amount of liquid in the flow goes to zero. 


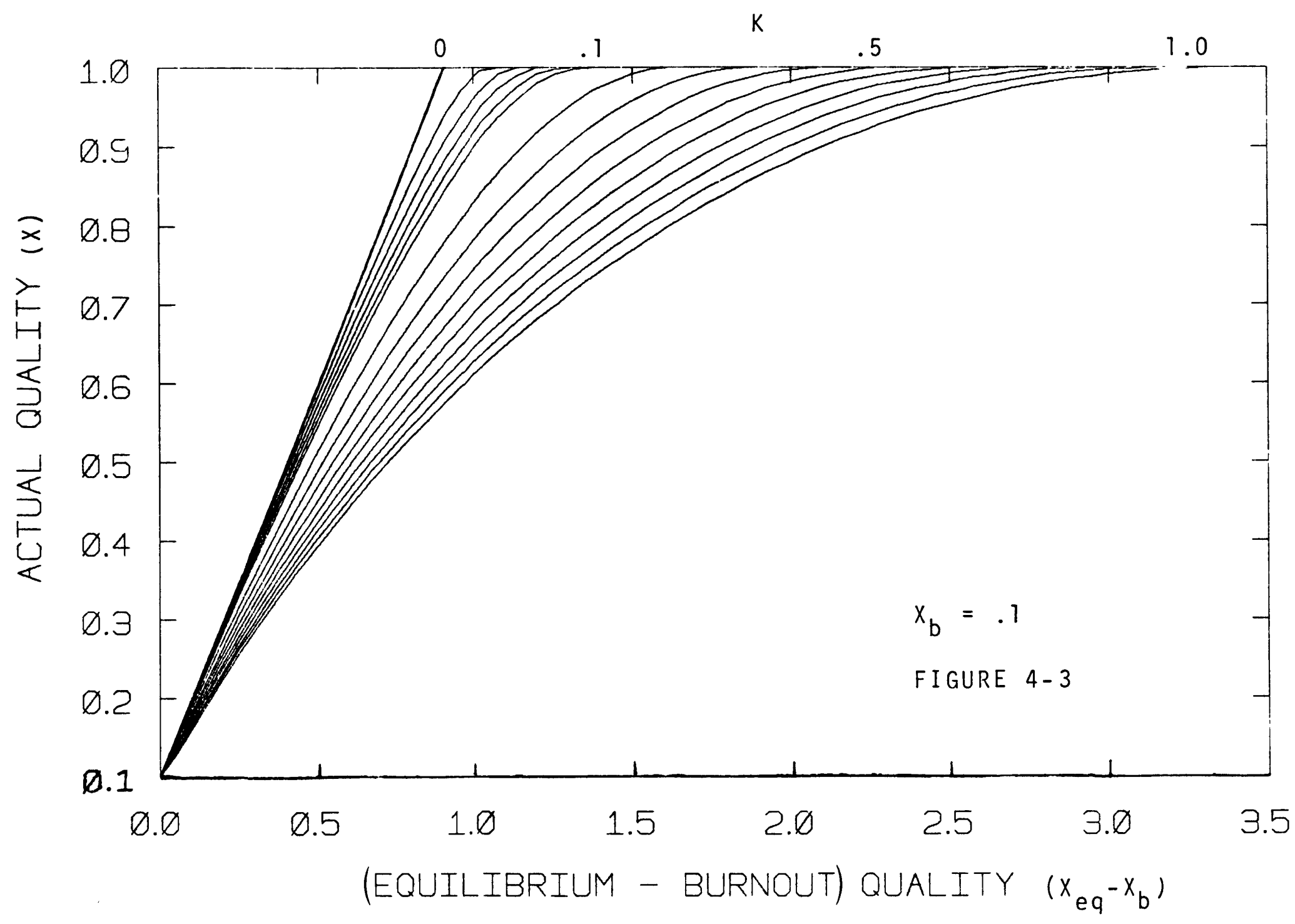




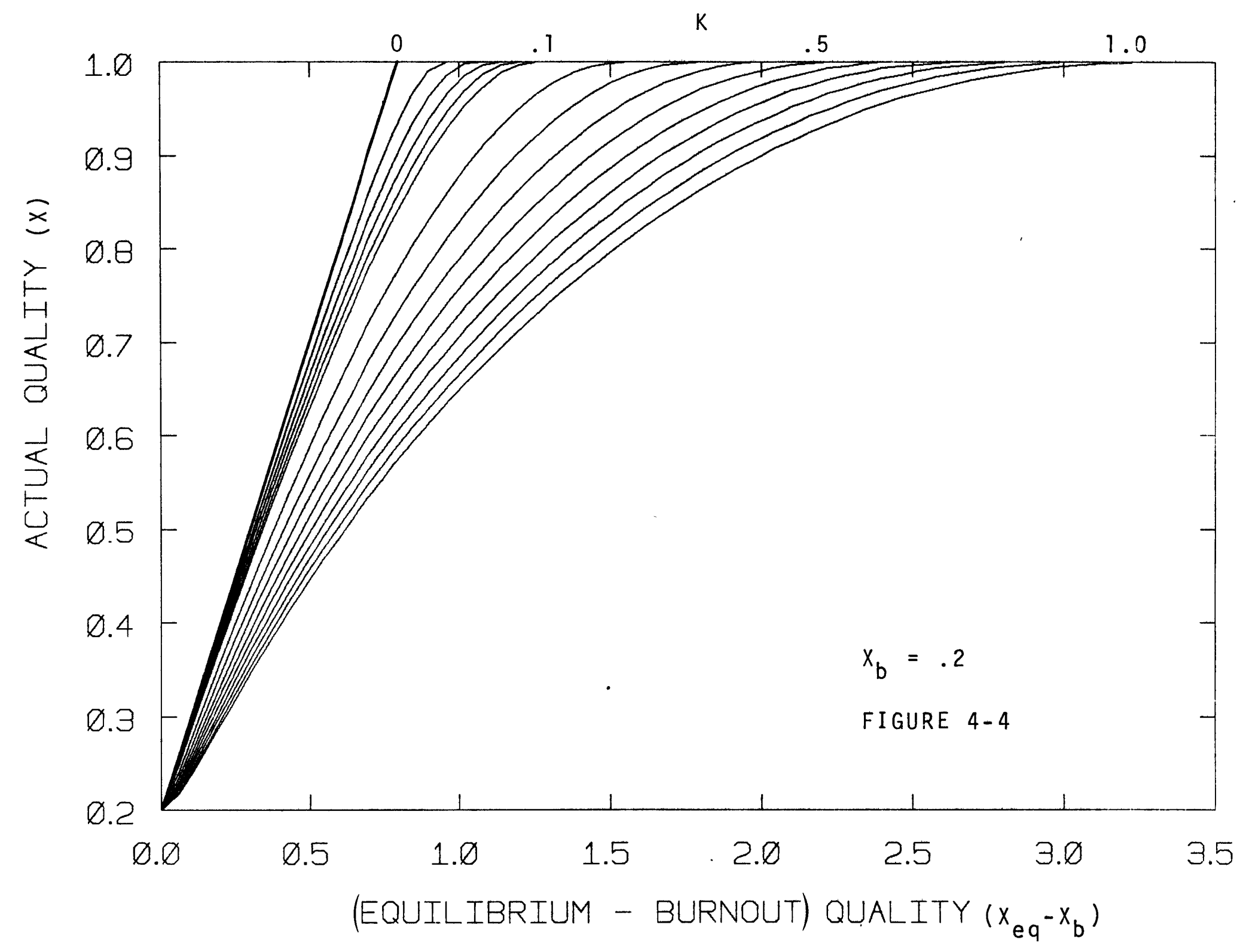




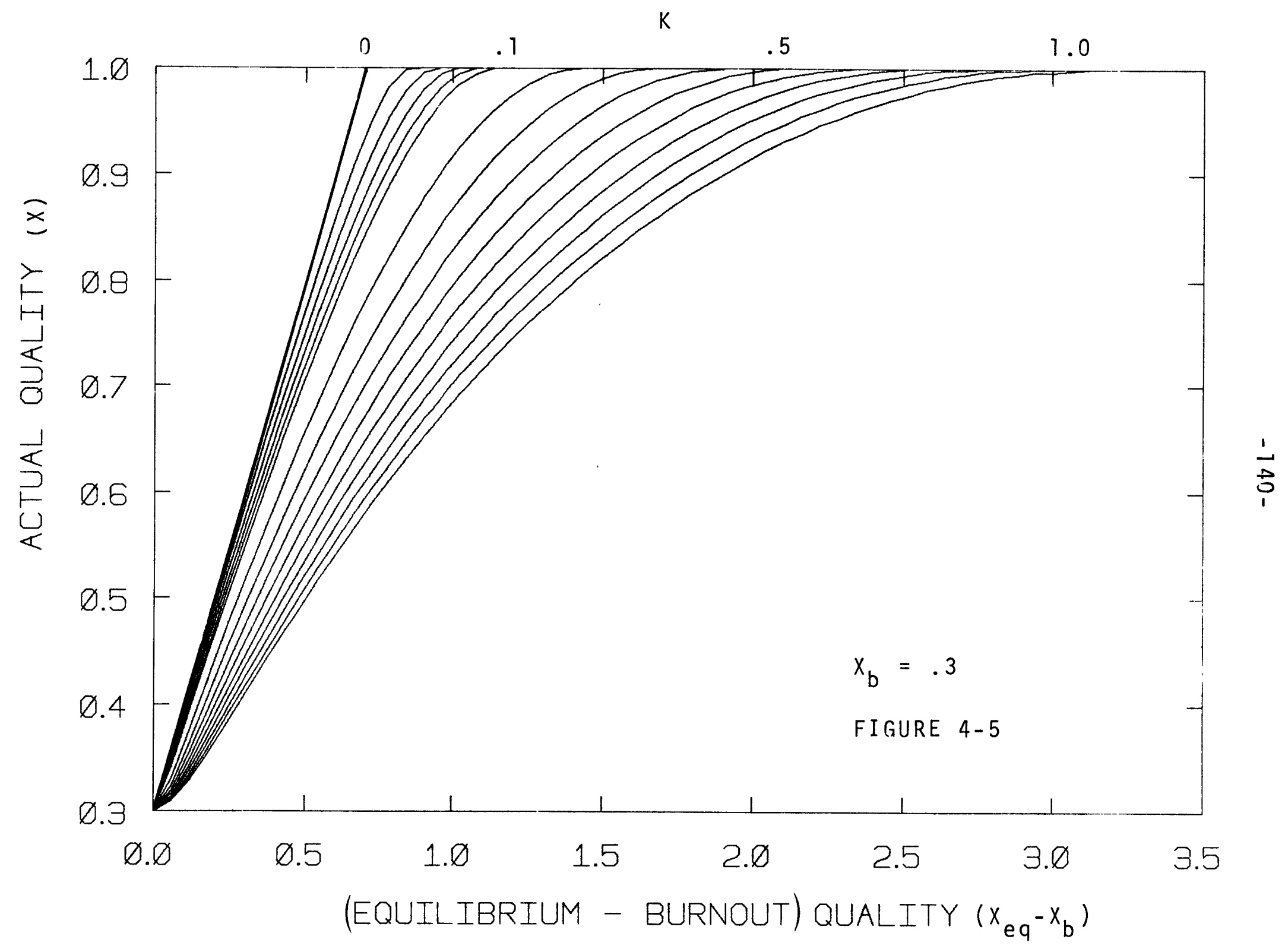




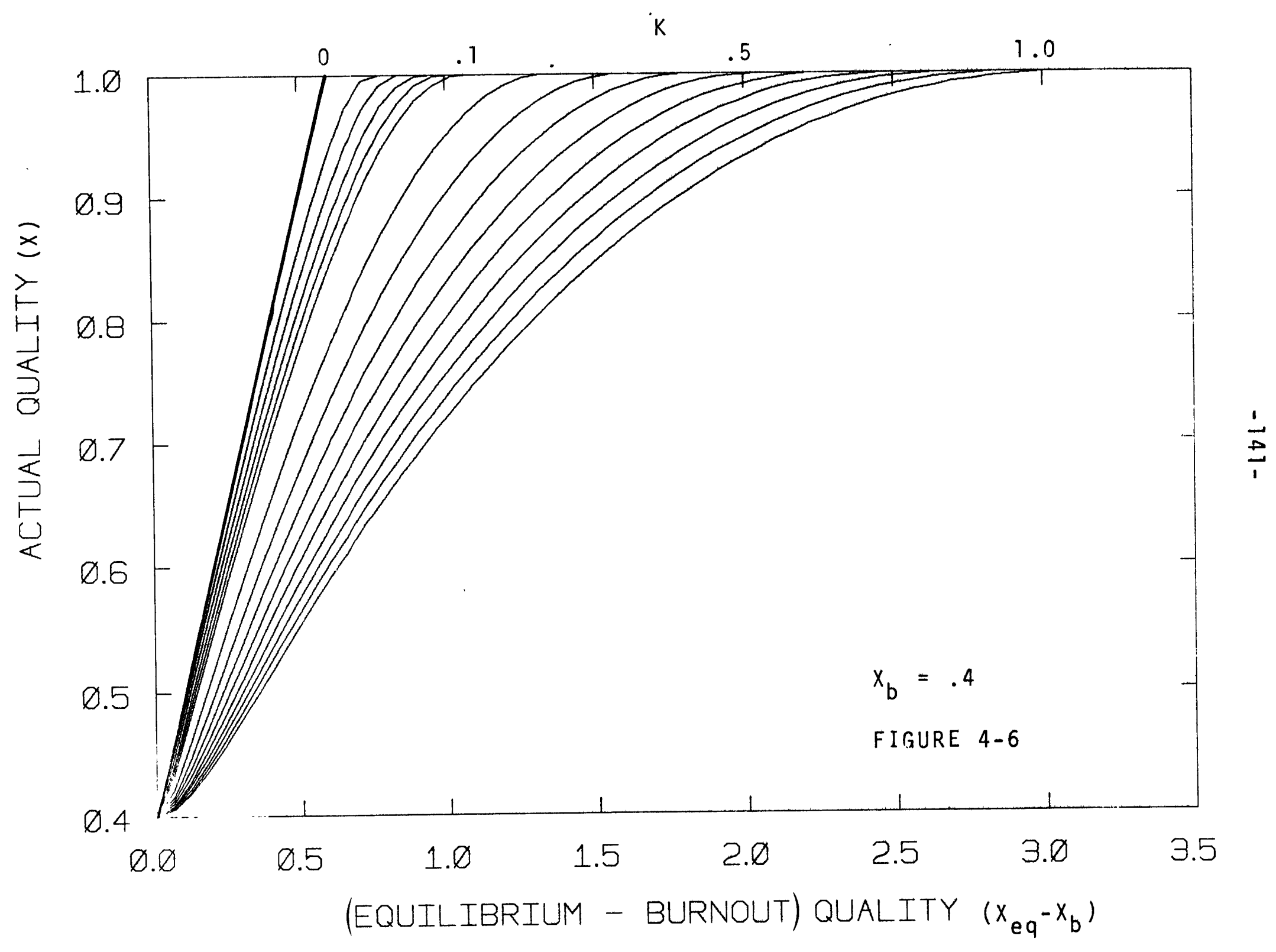




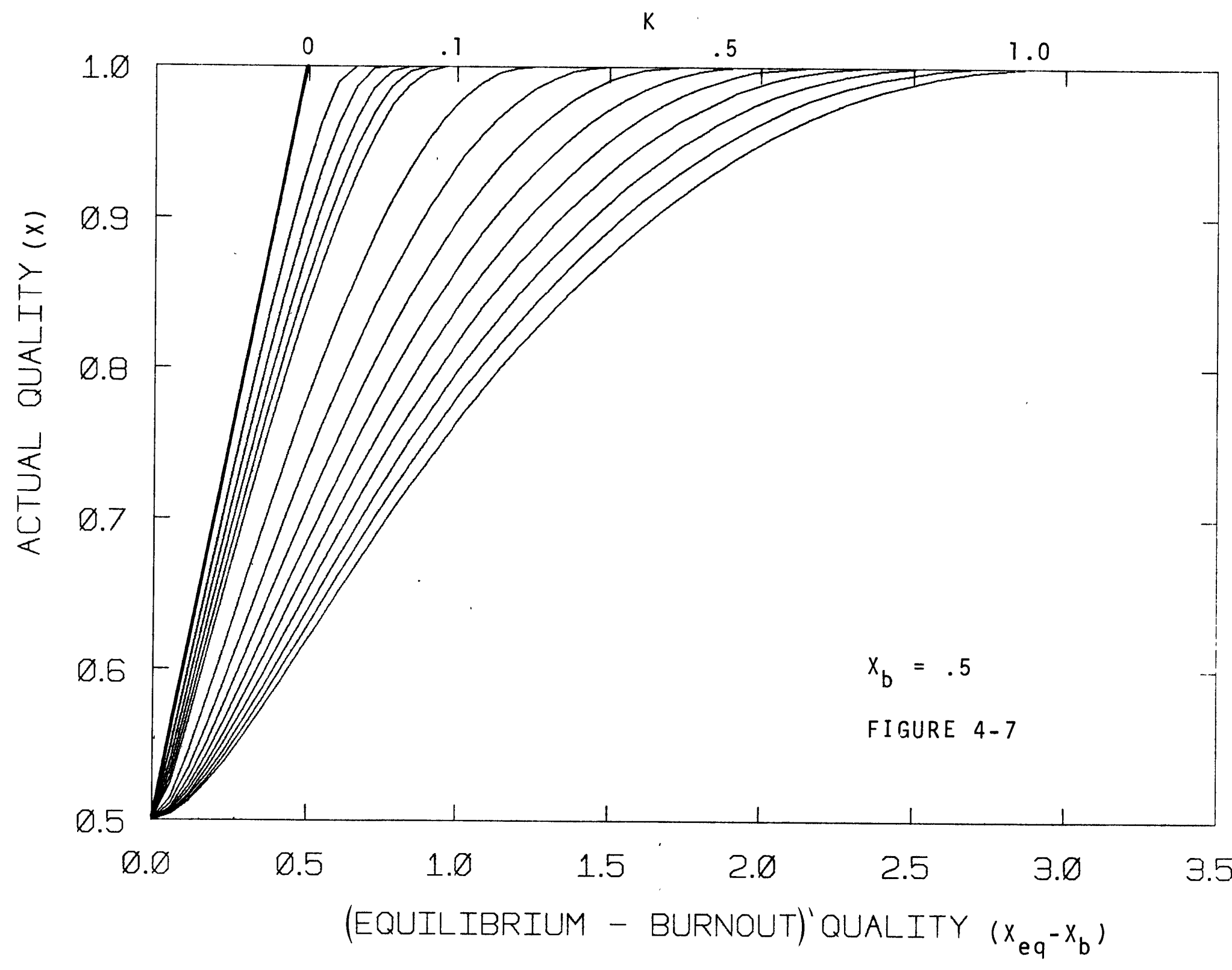

$\frac{1}{N}$ 


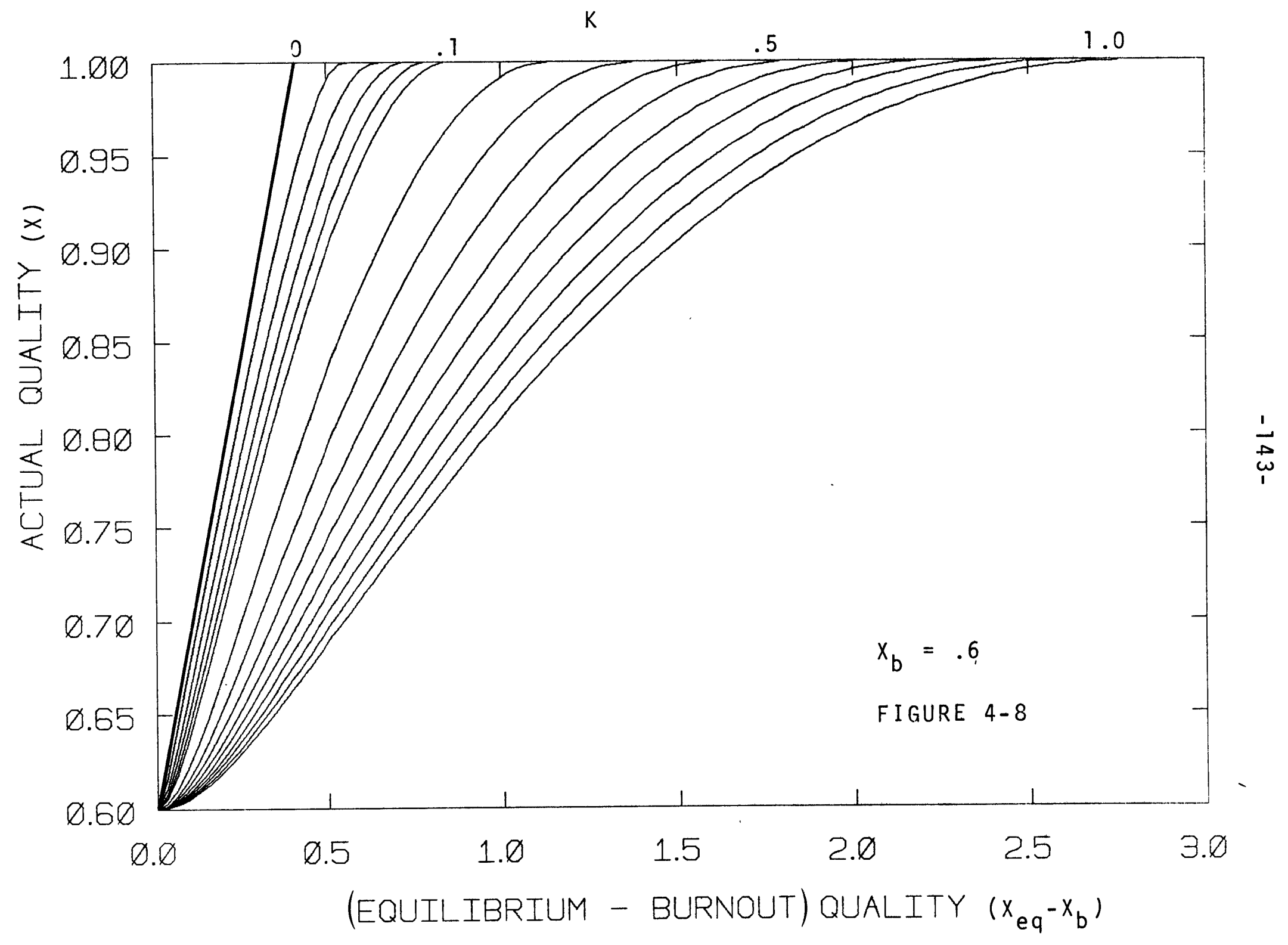




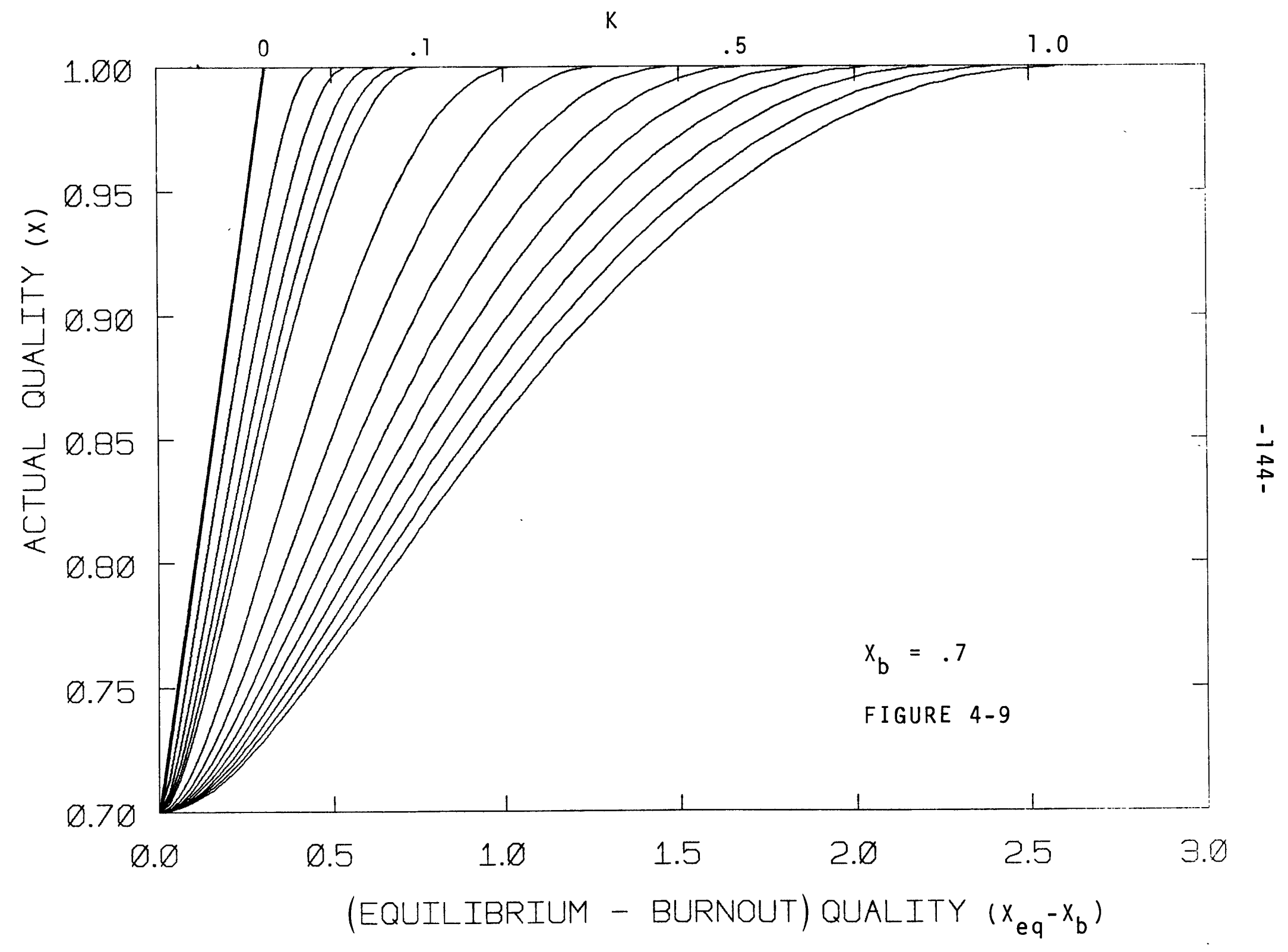




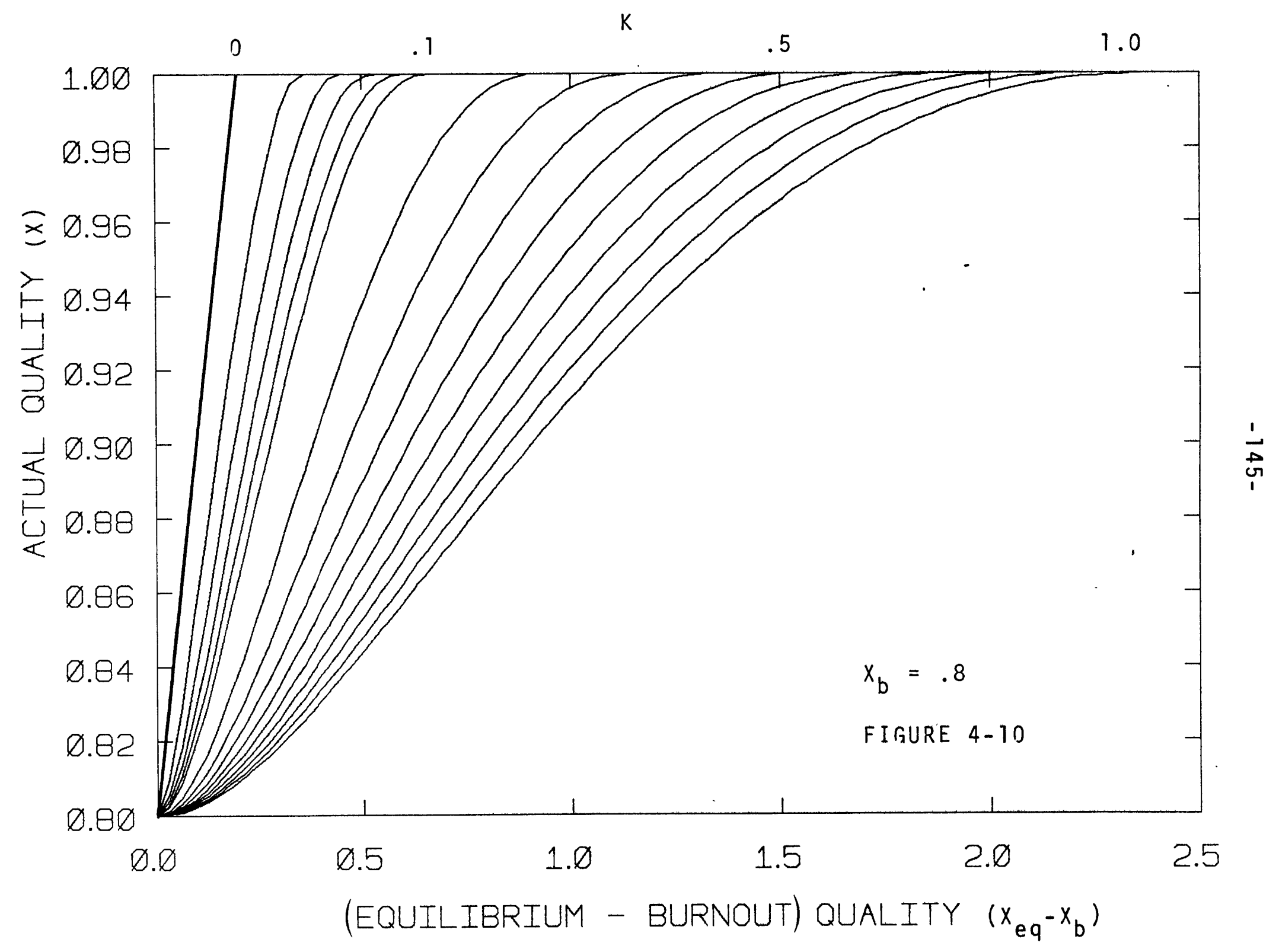




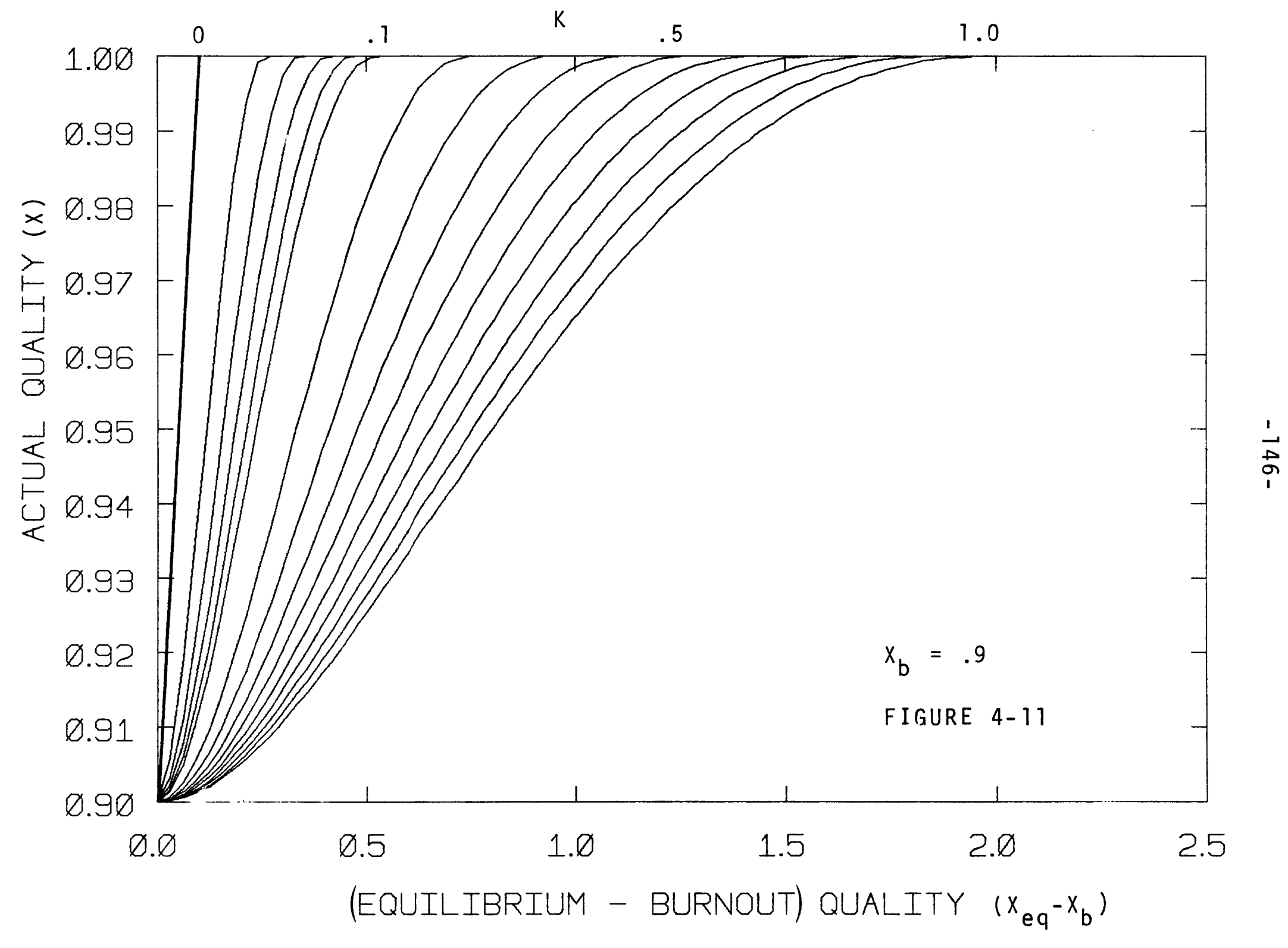


Conclusions from the numerical model show that the wall-to-drop term in Equation (3-28) is small compared to the total heat transfer to the fluid. Equation (3-28) can therefore be written,

$$
T_{w}-T_{v}=\frac{Q^{\prime \prime}}{h_{w}}
$$

The five governing equations presented in Chapter III $(3-4 a, 3-17,3-20,3-25,3-28)$ have been reduced to a set of equilibrium graphs (Equation (4-38)), an energy balance at the wall Equation (4-39), and slip Equations (4-6) or (4-7). Equations for the vapor and liquid velocities, Equations (2-5) and (2-6) along with the definition of void fraction, Equation (2-7) remain unchanged.

\subsection{Calculation Procedure}

A calculation procedure can now be established for calculating wall temperatures. The following equations will be referred to in the description of the procedure. 


$$
\begin{aligned}
& -148- \\
& S_{f}=\frac{v_{v}}{v_{\ell f}}=\left(\frac{\rho_{\ell}}{\rho_{v}}\right)^{.205}\left(\frac{G D_{T}}{\mu_{\ell}}\right)^{-0.016} \\
& v_{v}=\frac{G X}{\rho_{v}^{\alpha}} \\
& v_{\ell}=\frac{v_{v}}{s} \\
& \alpha=\frac{1}{\frac{\rho_{v}}{\rho_{\ell}} s \frac{1-x}{x}+1} \\
& \frac{D}{D_{T}}=\frac{1}{x_{b}-0.1}\left\{\frac{D_{b}}{D_{T}}\left(x_{a}-.1\right)+\left(\frac{\rho_{\ell}}{\rho_{v}}\right)^{2} \frac{W_{c}}{\left(S_{f}-1\right)^{2}} \frac{\rho_{v} \sigma g_{c}}{G^{2} D_{T}}\right. \\
& \left.\frac{1}{\left(\frac{\rho_{\ell}}{\rho_{v}} \frac{1}{S_{f}}-1\right)}\left[\frac{1}{1+x_{a}\left(\frac{\rho_{\ell}}{\rho_{v}} \frac{1}{S_{f}}-1\right)}-\frac{1}{1+x_{b}\left(\frac{\rho_{\ell}}{\rho_{v}} \frac{1}{S_{f}}-1\right)}\right]\right\} 2-18 \\
& \frac{\frac{\rho_{v}}{\rho_{\ell}}+\left(\frac{1}{s_{f}}-\frac{\rho_{v}}{\rho_{\ell}}\right) x_{a}}{\frac{\rho_{v}}{\rho_{\ell}}+\left(\frac{1}{s}-\frac{\rho_{v}}{\rho_{\ell}}\right) \times}=\frac{1}{s_{f}-1} \\
& N u_{w}=.023 \mathrm{Re}^{.8} \mathrm{Pr}^{.4}\left(1+\frac{.8 \mathrm{D}_{\mathrm{T}}}{\mathrm{z}}\right) \\
& N u_{w}=.0157 \mathrm{Re}^{.84} \mathrm{Pr}^{1 / 3}\left(\frac{D_{T}}{L_{D 0}}\right)^{.04} \quad 6<\frac{L_{D 0}}{D_{T}}<60 \text { Heineman } \\
& N u_{w}=.0133 \mathrm{Re}^{.84} \mathrm{Pr}^{1 / 3} \quad \frac{\mathrm{L}_{\mathrm{D} 0}}{\mathrm{D}_{\mathrm{T}}}>60
\end{aligned}
$$




$$
\begin{aligned}
& \tau=\frac{\rho_{v} \sigma g_{C}}{G^{2} D_{T}} \\
& \text { surface tension group 4-1 } \\
& A c=\frac{Q^{\prime \prime}}{G h_{f g}} \\
& G r=\frac{\rho_{v}\left(\rho_{\ell}-\rho_{V}\right) D_{T} g}{G} \quad \text { Gravity group } \\
& s=\left[1+\left\{\left(1-\frac{4}{3} G r \frac{\alpha^{2}}{x^{2}} \frac{1}{C_{D}} \frac{D}{D_{T}}\right)\left(\frac{16}{3} \frac{\rho_{\ell}}{\rho_{V}} A c \frac{1}{x C_{D}} \frac{D}{D_{T}}-1\right)+1\right\}^{1 / 2}\right] / \\
& {\left[1-\frac{4}{3} \operatorname{Gr} \frac{\alpha^{2}}{x^{2}} \frac{1}{C_{D}} \frac{D}{D_{T}}\right]} \\
& s-1=\left(\frac{16}{3} \frac{\rho_{\ell}}{\rho v} A c \frac{1}{X_{b} C_{D}} \frac{D_{b}}{D_{T}}\right)^{1 / 2} \\
& \frac{D_{0}}{D_{T}}=\frac{1}{1-X_{b}}\left[\frac { 3 \sqrt { \tau W e } C _ { D } } { 8 } \frac { \rho _ { V } } { \rho _ { \ell } } \left\{\left(\frac{16}{3} A C \frac{\rho_{\ell}}{\rho_{V}} \frac{X_{T A}}{C_{D}}+\frac{4}{3} \frac{G r}{C_{D}}\right)^{1 / 2}-\right.\right. \\
& \left.\left.\left(\frac{16}{3} A c \frac{\rho_{\ell}}{\rho_{V}} \frac{X_{b}}{C_{D}}+\frac{4}{3} \frac{G r}{C_{D}}\right)^{1 / 2}\right\}+\left(1-X_{T A}\right) \frac{D_{T A}}{D_{T}}\right] \\
& x_{T A}=\frac{3}{5}-\frac{1}{10} \frac{G r}{A C} \frac{\rho_{v}}{\rho_{\ell}} \\
& \frac{D_{T A}}{D_{T}}=\left[3 \tau W_{C} C_{D} /\left(16 A c \frac{\rho_{\ell}}{\rho_{V}} x_{T A}+4 G r\right)\right]^{1 / 2} \\
& \frac{D_{b}}{D_{T}}=\left[\begin{array}{lll}
3 \tau W_{C} & \left.C_{D} /\left(16 A c \frac{\rho_{\ell}}{\rho_{v}} X_{b}+4 G r\right)\right]^{1 / 2} 4-12
\end{array}\right.
\end{aligned}
$$




$$
\begin{aligned}
& -150- \\
& \frac{D_{b \max }}{D_{T A}}=\left[\frac{1-x_{b}}{1-x_{T A}}\right]^{1 / 3} \\
& \begin{array}{l}
X_{I}=\frac{3}{16} \frac{C_{D}}{A c} \frac{\rho_{V}}{\rho_{\ell}}\left[\left(\frac{D_{T}}{\bar{D}}\right)^{2} \tau W_{C}-\frac{4}{3} \frac{G r}{C_{T}}\right] \\
\frac{1}{-X_{b}}\left[\frac{\bar{D}}{D_{T}}\left(X_{I}-X_{b}\right)+\frac{D_{T A}}{D_{T}}\left(1-X_{T A}\right)+\frac{3}{8} \frac{\sqrt{\tau W_{C}} C_{D}}{A c} \frac{\rho_{V}}{\rho_{\ell}}\right.
\end{array} \\
& \left.\left\{\left(\frac{16}{3} A C \frac{\rho_{\ell}}{\rho_{V}} \frac{X_{T A}}{C_{D}}+\frac{4}{3} \frac{G r}{C_{D}}\right)^{1 / 2}-\left(\frac{16}{3} A C \frac{\rho_{\ell}}{\rho_{V}} \frac{X_{I}}{C_{D}}+\frac{4}{3} \frac{G r}{C_{D}}\right)^{1 / 2}\right\}\right] \quad 4-15 \\
& x_{e q}-X_{b}=\frac{4 Q^{\prime \prime}}{G h_{f g}} \frac{z}{D_{T}} \\
& \operatorname{Re}_{D}^{\prime}=\frac{\rho_{v} V_{\ell} D}{\mu_{v}} \\
& \frac{C_{p}}{h_{f g}}\left(T_{v}-T_{s}\right)=\frac{x_{e q}}{x}-1 \\
& N u_{0} \cong 2+.6\left[\frac{\rho_{v}\left(v_{v}-v_{\ell}\right) D}{\mu_{v}}\right]^{1 / 2} \operatorname{Pr}_{v}{ }^{1 / 3} \quad 4-29 \\
& K=\frac{2}{3} \frac{D_{0}}{D_{T}} \frac{1}{\left(1-x_{b}\right)^{1}} 1 / 3 \frac{Q^{\prime \prime}}{G h_{f g}} \operatorname{Pr}_{v} \frac{\rho_{\ell}}{\rho_{v}}\left[\frac{R e_{D}^{\prime}}{N u_{0}} \frac{1}{x^{3 / 4}(1-x)^{1 / 12}}\right] \quad 4-37 \\
& T_{w}-T_{v}=\frac{Q^{\prime \prime}}{h_{w}}
\end{aligned}
$$


Calculations should be performed assuming a value of $C_{D}=.4$. In all cases examined, this was a valid assumption, however, it can be checked once a value for $\operatorname{Re}_{D}$ is determined. If $\operatorname{Re}_{D}>150, C_{D}=.4$. If $\operatorname{Re}_{D}<150$, $C_{D}$ can be calculated using Equation (3-34).

4-2a Calculation of Initial Drop Size at $x=X_{b}$ It is easiest to separate initial drop size calculations for inverted annular and annular flows.

1. Inverted Annular Flow

Step Number

$\begin{array}{lc}1 & \tau, A c, G r \\ 2 & X_{T A} \\ 3 & D_{T A} \\ 4 & D_{0}\end{array}$

2. Annular Flow
Equation Number

$(4-1),(4-2),(4-3)$

$(4-10)$

$(4-11)$

$(4-9)$

$\begin{array}{ccc}\text { Step Number } & \text { Calculation } & \text { Equation Number } \\ 1 & \tau, \text { Ac, Gr } & (4-1),(4-2),(4-3) \\ 2 & D_{b} & (4-12) \\ 3 & S & (4-6) \text { or }(4-7 a) \\ 4 & S_{f} & (2-4)\end{array}$




$\begin{array}{ccc}\text { Step Number } & \text { Calculation } & \text { Equation Number } \\ 5 & \mathrm{X}_{\mathrm{a}} & (2-19) \\ 6 & \bar{D} & (2-18)\end{array}$

For most cases, $D_{0}=\bar{D}$, however, a check can be made to determine if the drops will break up beyond burnout.

$\begin{array}{ccc}\text { Step Number } & \text { Calculation } & \text { Equation Number } \\ 7 & \mathrm{X}_{\text {TA }} & (4-10) \\ 8 & D_{\text {TA }} & (4-11) \\ 9 & D_{b \max } & (4-13)\end{array}$
If $D_{b \max }>\bar{D}$ then no drop breakup occurs, and $D_{0}=\bar{D}$, calculated in step 6 . If $D_{b \max }<\bar{D}$ then break- up occurs and further calculation is necessary.

$\begin{array}{ccc}\text { Step Number } & \text { Calculation } & \text { Equation Number } \\ 10 & X_{I} & (4-14) \\ 11 & D_{0} & (4-15)\end{array}$


4-2b Calculation of Constant $k$

Calculations are performed with $x=x_{b}$ and $D=D_{0}$.

$\begin{array}{ccc}\text { Step Number } & \text { Calculation } & \begin{array}{c}\text { Equation Number } \\ 1\end{array} \\ 2 & S & (4-6) \text { or }(4-7 a) \\ 3 & \alpha & (2-7) \\ 4 & v_{v}, v_{\ell} & (2-5),(2-6) \\ 5 & \mathrm{Nu}_{0} & (4-29) \\ 6 & \mathrm{Re}_{D}^{\prime} & (4-22) \\ \mathrm{K} & (4-37)\end{array}$

A considerable simplification of these six steps is possible if the gravity criterion, $\frac{10}{3}$ Gr $\frac{1}{X_{b}}{ }^{2} \frac{D_{0}}{D_{T}} \ll 1$ holds. In this case, free stream slip is given by Equation (4-7a). If $\mathrm{Nu} \mathrm{u}_{0}$, Equation (4-29) is assumed large (i.e. 15 or greater). Then the equation for $K$, Equation (4-37) can be rearranged and written as

$$
\begin{gathered}
K \cong .58\left(\frac{D_{0}}{D_{T}}\right)^{1.25} \frac{1}{\left(1-x_{b}\right)^{5 / 12}}\left[\frac{Q^{\prime \prime}}{G h_{f g}}\right]^{.75}\left[\frac{\rho_{\ell}}{\rho_{v}}\right]^{.75}\left[\frac{G D_{T}}{\mu_{v}}\right]^{1 / 2} \\
\operatorname{Pr}_{v}^{2 / 3}\left[\frac{\rho_{v}}{\rho_{\ell}} \frac{\left(1-x_{b}\right)}{x_{b}}+\frac{1}{1+3.65 \sqrt{\frac{\rho_{\ell}}{\rho_{v}} \frac{Q^{\prime \prime}}{G h_{f g}} \frac{D_{0}}{D_{T}} \frac{1}{X_{b}}}}\right]^{1 / 2} 4-40
\end{gathered}
$$


Equation (4-40) predicted values of $K$ to within about $15 \%$ of those predicted using steps 1 through 6 above for applicable flow conditions investigated in this study. A comparison. of $K$ predicted by Equation(4-40) and the six steps above is given in Table 4-1.

$\begin{array}{cccc}\text { F1uid mass } \mathrm{flux} \frac{\mathrm{lbm}}{\mathrm{ft} \mathrm{t}^{2} \mathrm{hr}} & \text { Equation }(4-40) & \text { Steps } 1-6 \\ \mathrm{H}_{2} \mathrm{O} & 3.8 \times 10^{6} & 0.0194 & 0.023 \\ & 2.9 \times 10^{5} & 2.4 & 2.3 \\ & 1 \times 10^{6} & 0.24 & 0.22 \\ & 7.49 \times 10^{5} & .4 & 0.366 \\ \mathrm{Fr}-12 & 6.18 \times 10^{5} & 0.476 & 0.446 \\ & 7.66 \times 10^{5} & 0.416 & 0.395 \\ & 4.9 \times 10^{5} & 0.58 & 0.52 \\ & 1.5 \times 10^{6} & 0.0737 & 0.0714\end{array}$




\section{2c Calculation of Local Wall Temperature}

$\begin{array}{ccc}\text { Step Number } & \text { Calculation } & \text { Equation Number } \\ 1 & x_{e q} & (4-21) \\ 2 & x & \text { use graphs for ap- } \\ & & \text { propriate } x_{b} \text { and } K \\ 3 & T_{v} & \text { Figs } .4-3, \text { thru } 4-11\end{array}$

For most cases, the local void fraction is high and can be assumed equal to 1 , however, it is possible with existing information to calculate the local slip and void fractions using equations (4-6) or (4-7) and Equation (2-7) evaluated at the local conditions.

$\begin{array}{cc}\text { Step Number } & \text { Calculation } \\ 4 & \operatorname{Re}=\frac{G X D_{T}}{\mu_{V} a} \\ 5 & h_{W} \\ 6 & T_{W}\end{array}$

Equation Number
(3-49) or Heineman $(4-39)$

A sample calculation for annular flow is given in Appendix A-4. 


\subsection{Local Conditions Solution and Results}

The local conditions model has been compared to data from five investigators and four different fluids.

\section{3a Comparison of the Local Conditions Model with Data}

FORSLUND'S NITROGEN DATA [3]

Figures 4-12 and 4-13 compare the local conditions solution to Forslund's nitrogen data. Calculated values of the constant $K$ are shown in these figures. For most of the nitrogen cases, the non-equilibrium in the flow is high (i.e. $K$ is high). The local model predicts wall temperatures which agree well with both the numerical solution and data. Again, predicted wall temperatures are high near burnout, probably because the flow regime near burnout is not dispersed, but inverted annular.

The local conditions solution predicts wall temperatures slightly higher than the step-wise analytical solution near burnout. The local conditions solution does not include conduction and drop-wall interactions. These mechanisms have the largest effect near burnout, and tend to decrease wall temperatures.

BENNETT'S WATER DATA [2]

Figures 4-14 and 4-15 compare the local model to 


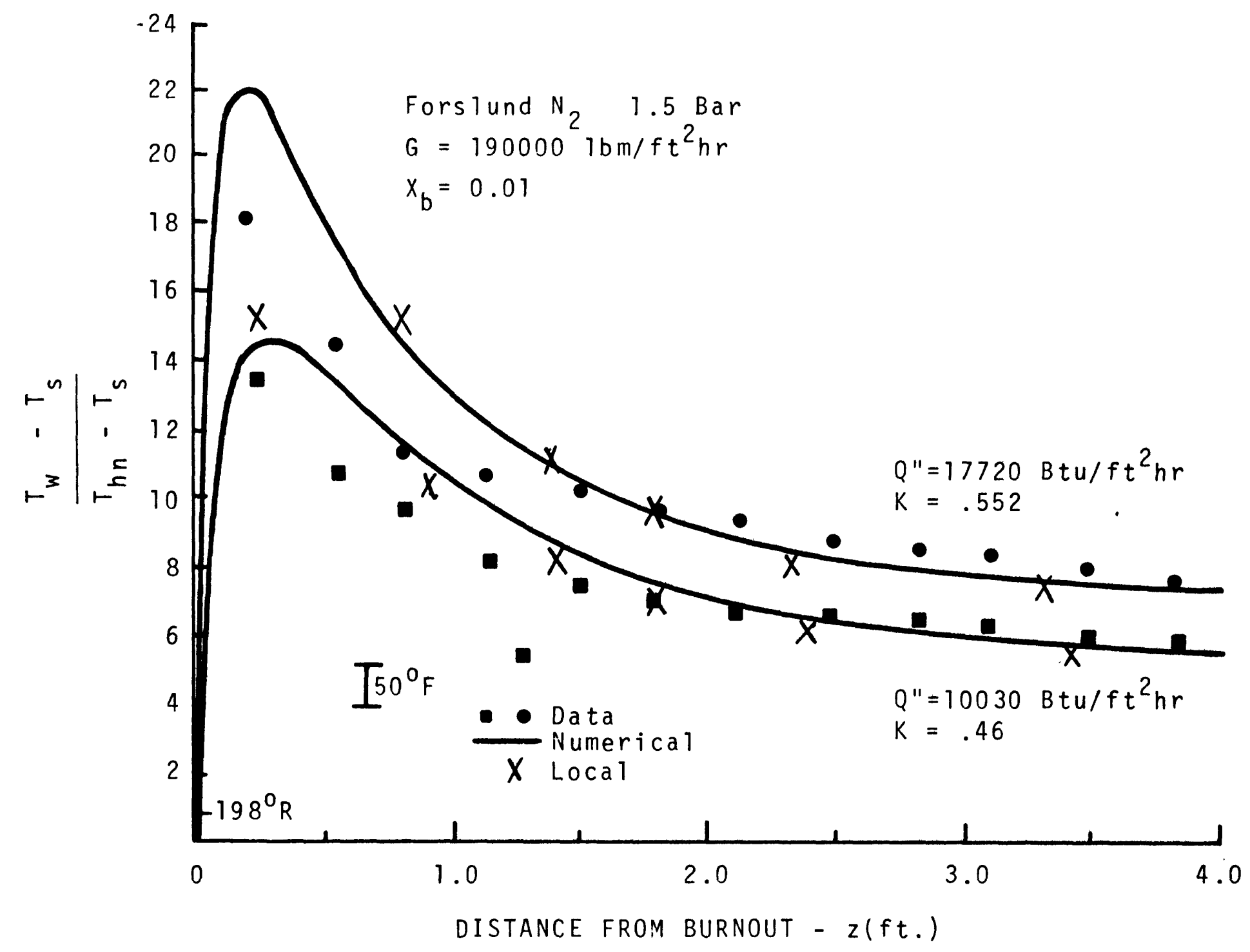

FIGURE 4-12 


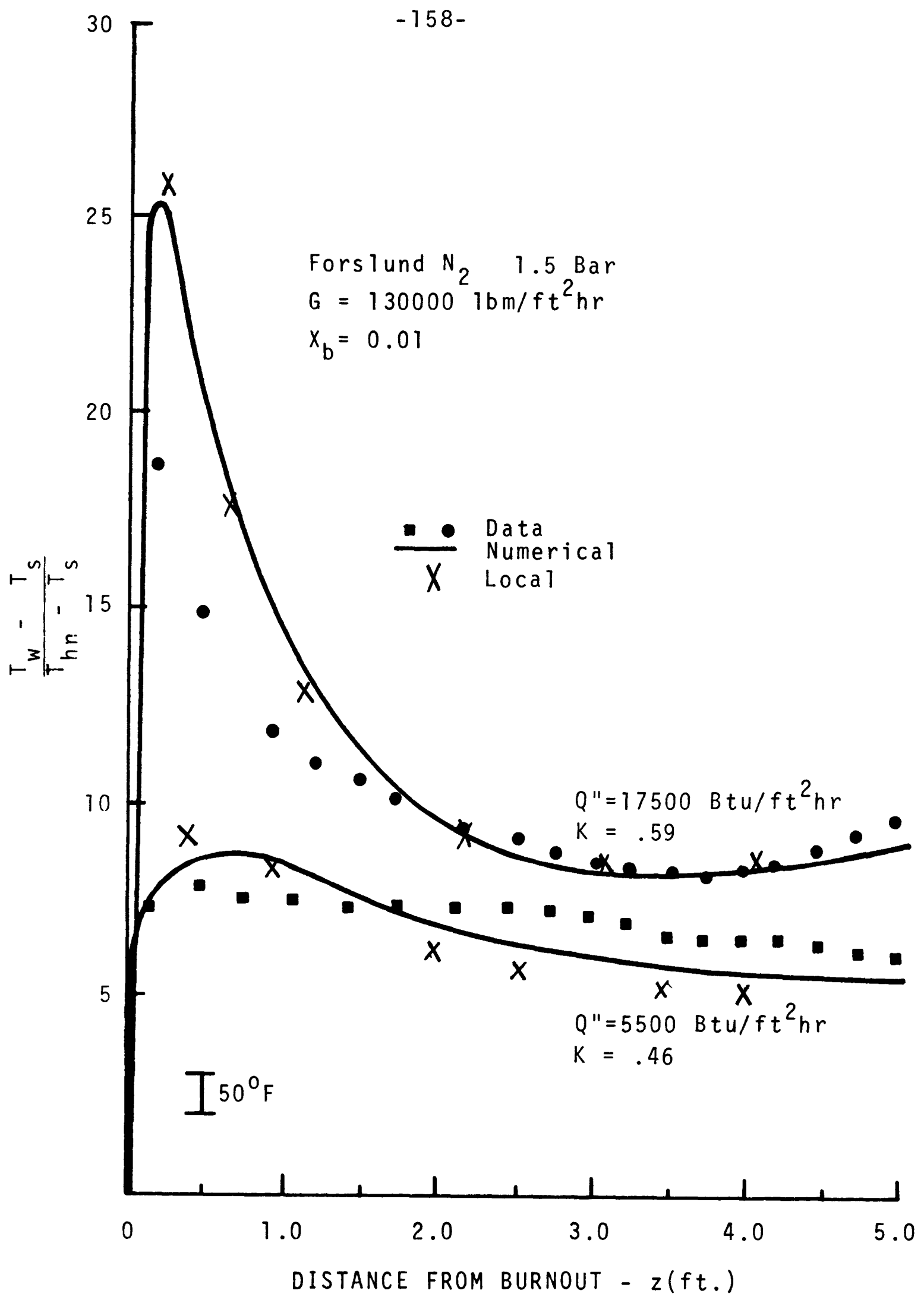

FIGURE 4-13 


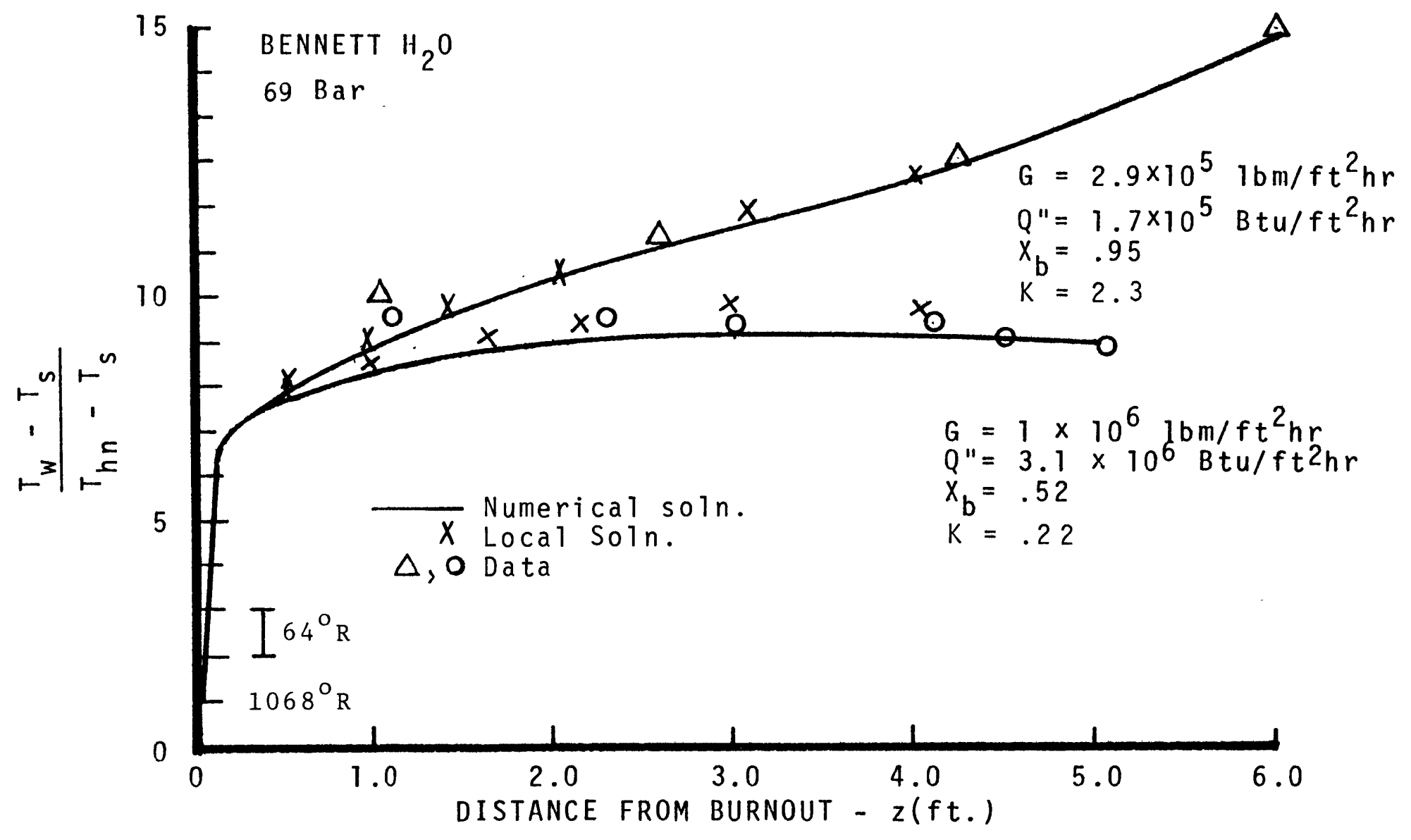

FIGURE 4-14 


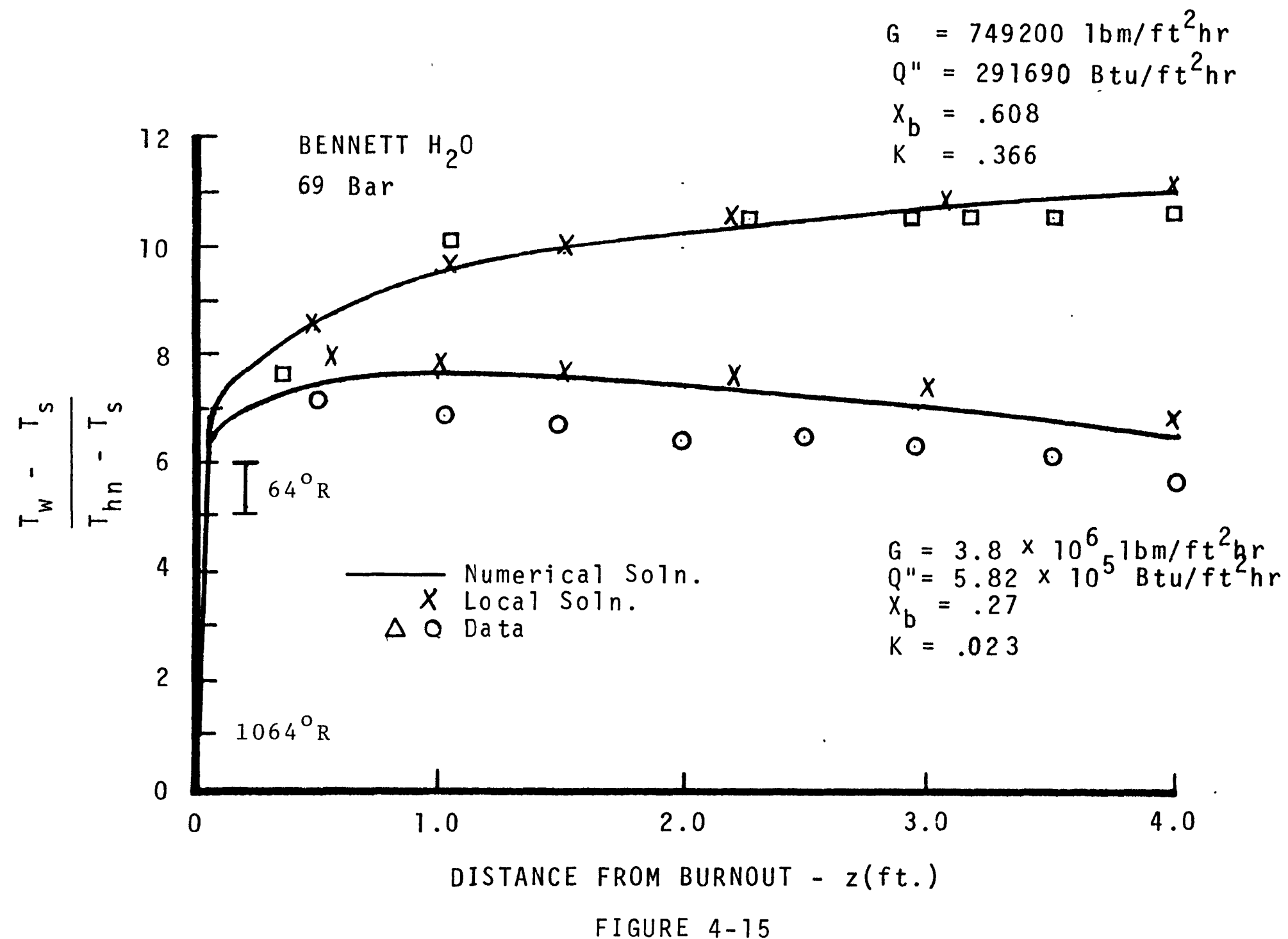

FIGURE 4-15 
water data. Again, agreement with both the numerical analytic solution and the data is good. Values of the constant $K$ range from an almost equilibrium case $(K=.023)$ for $G=3.8 \times 10^{6}$ in Figure 4-15 to a highly non-equilibrium case $(K=2.3)$ for $G=2.9 \times 10^{5}$ in Figure 4-14.

Because the local conditions model uses the significant heat transfer mechanisms of the numerical model, it cannot predict wall temperatures better than the computer solution. Thus, predicted wall temperatures are still high in Figure $4-15, G=3.8 \times 10^{6} 1 \mathrm{bm} / \mathrm{ft}^{2} \mathrm{hr}$.

\section{GROENEVELD'S FREON-12 DATA [5]}

Figure 4-16 is a comparison of Groeneveld's Freon-12 data to the local conditions solution. This model predicts wall temperatures which agree well with the Freon-12 data and the numerical solution. The $1.5 \times 10^{6} \frac{1 \mathrm{bm}}{\mathrm{ft}^{2} \mathrm{hr}}$ case is very close to equilibrium $(K=.0714)$, while in the $G=4.9 \times 10^{5} \frac{1 \mathrm{bm}}{\mathrm{ft} 2 \mathrm{hr}}$ case, mora non-equilibrium is present $(K=.5 \varepsilon)$.

CUMO'S FREON-12 DATA [6]

Figures 4-17 and 4-18 compare the local solution to Cumo's Freon-12 data. The local conditions wall tempera- 


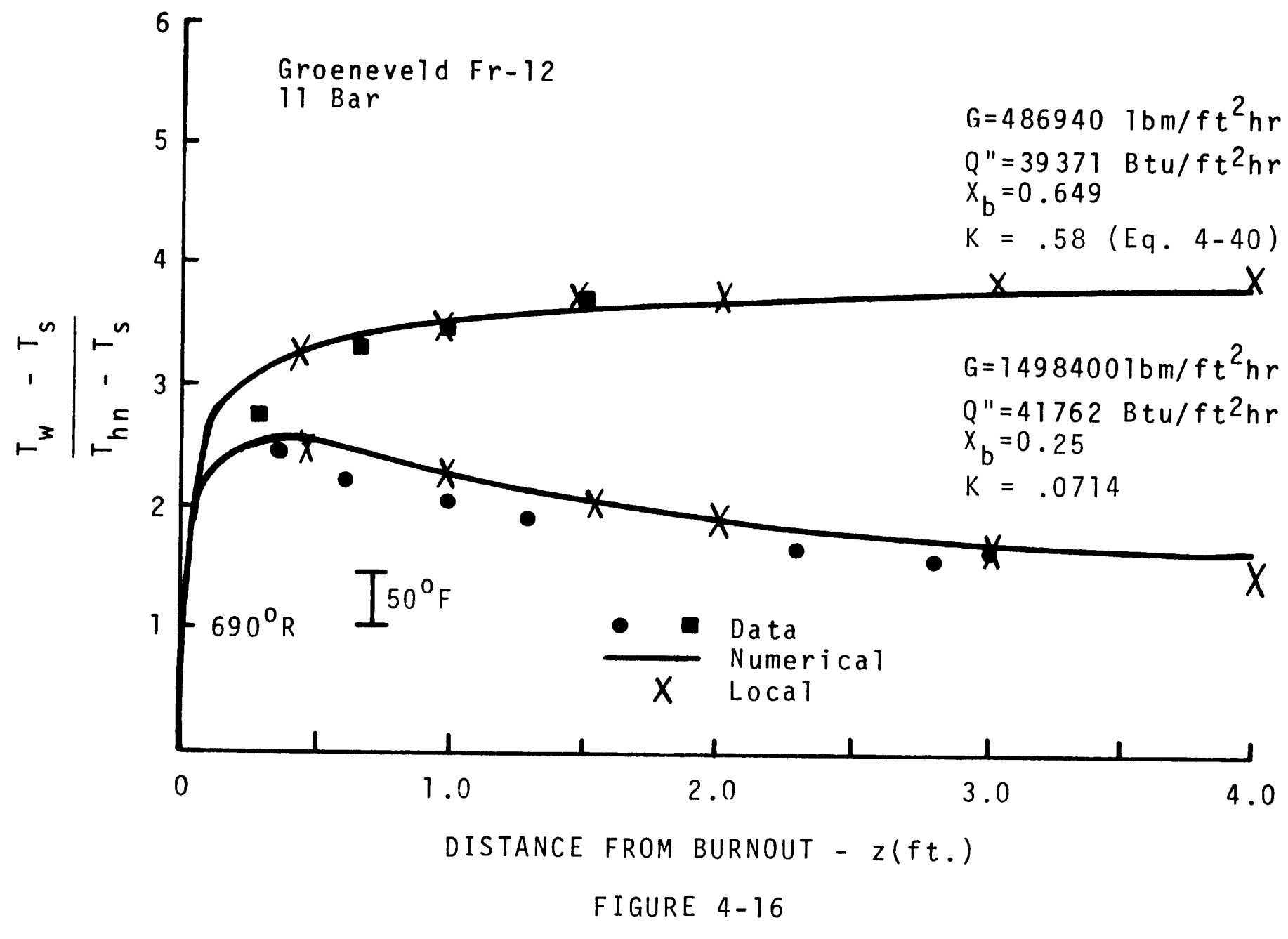




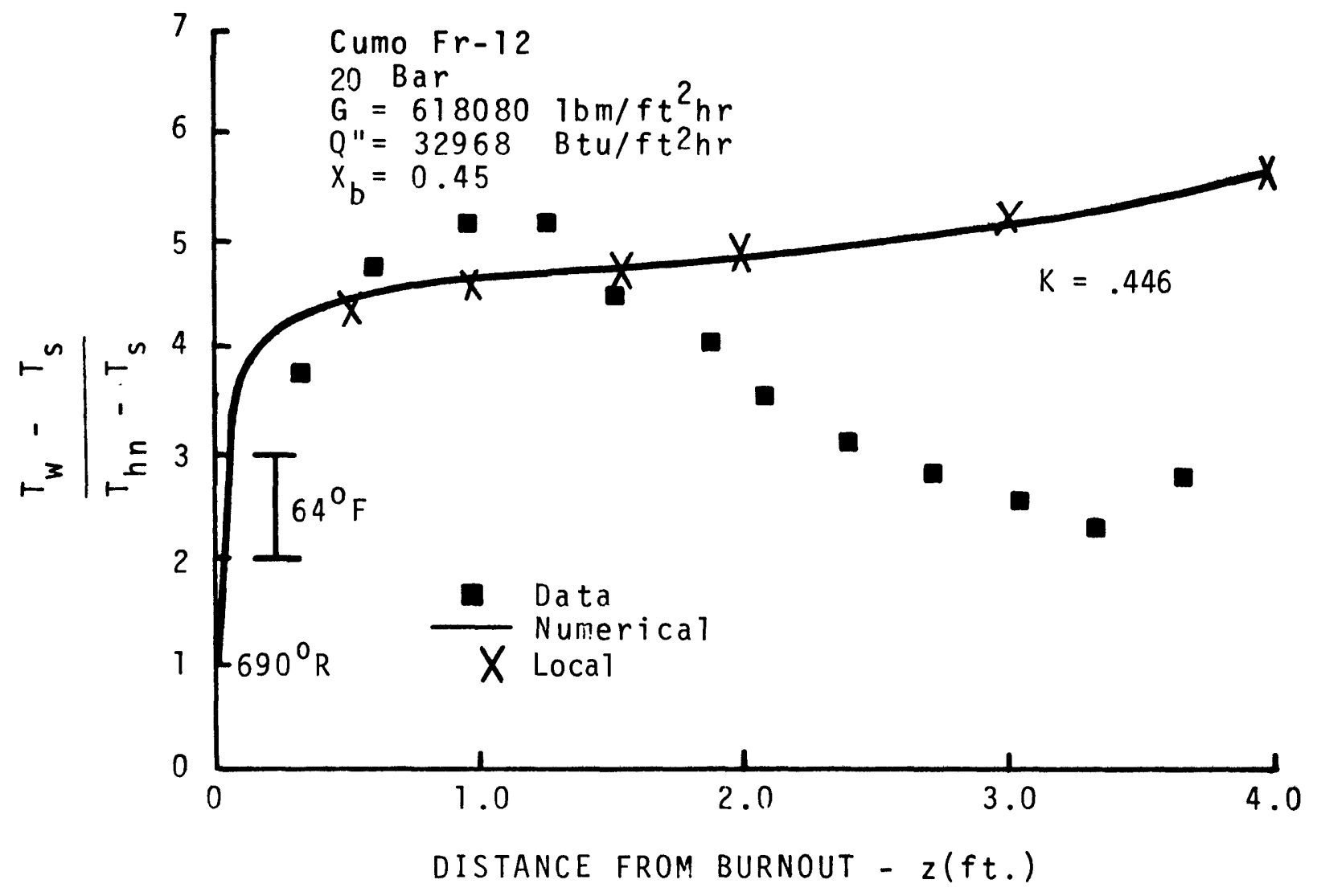

FIGURE 4-17 


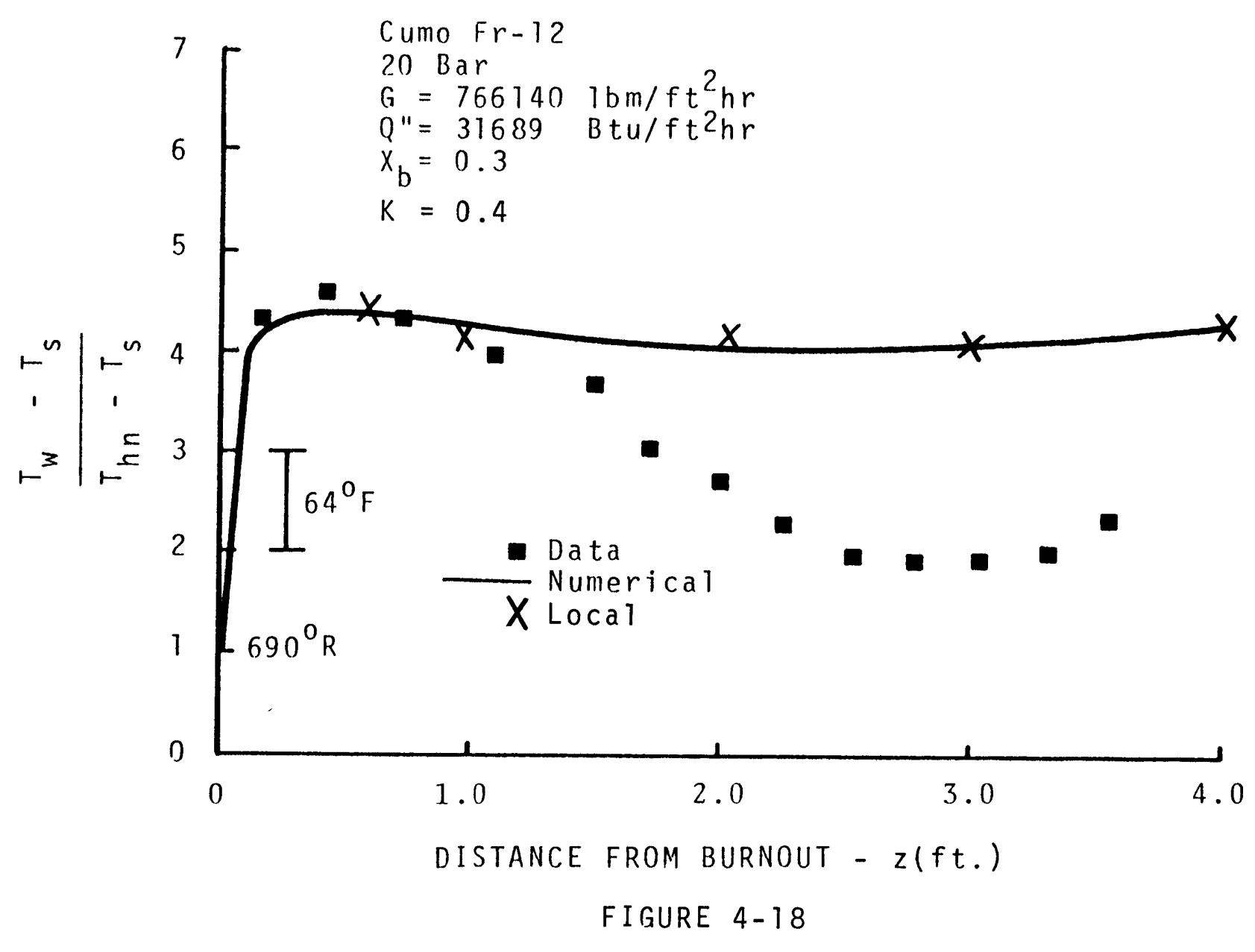


ture prediction agrees well with the numerical solution, however, no improvement is seen in comparison with wall temperature data. The predicted amount of non equilibrium present is approximately the same in both cases $(K=.395$ in Figure $4-18$ and $K=.446$ in Figure 4-17).

\section{UEDA'S FREON-113 DATA [8]}

Ueda's Freon-113 Data is compared with the local solution in Figures 4-19 and 4-20. His data was taken using an apparatus similar to that sketched in Figure 4-21. Both vapor and saturated liquid are fed into an unheated section where an annular flow develops. Droplets are entrained from the annular film and flow up the tube. The liquid film is removed in the film separator, and the remainder of the flow travels into the heated test section. A liquid film reforms on the test section wall due to droplet deposition, until dryout occurs.

Drops formed in the initial unheated length might be expected to have a diameter characterized by a Helmholtz instability analysis, while droplets formed from the film in the test section might be expected to have a diameter characterized by the film slip Weber number mechanism.

Figures 4-19 and 4- 20 both show two predicted wall 


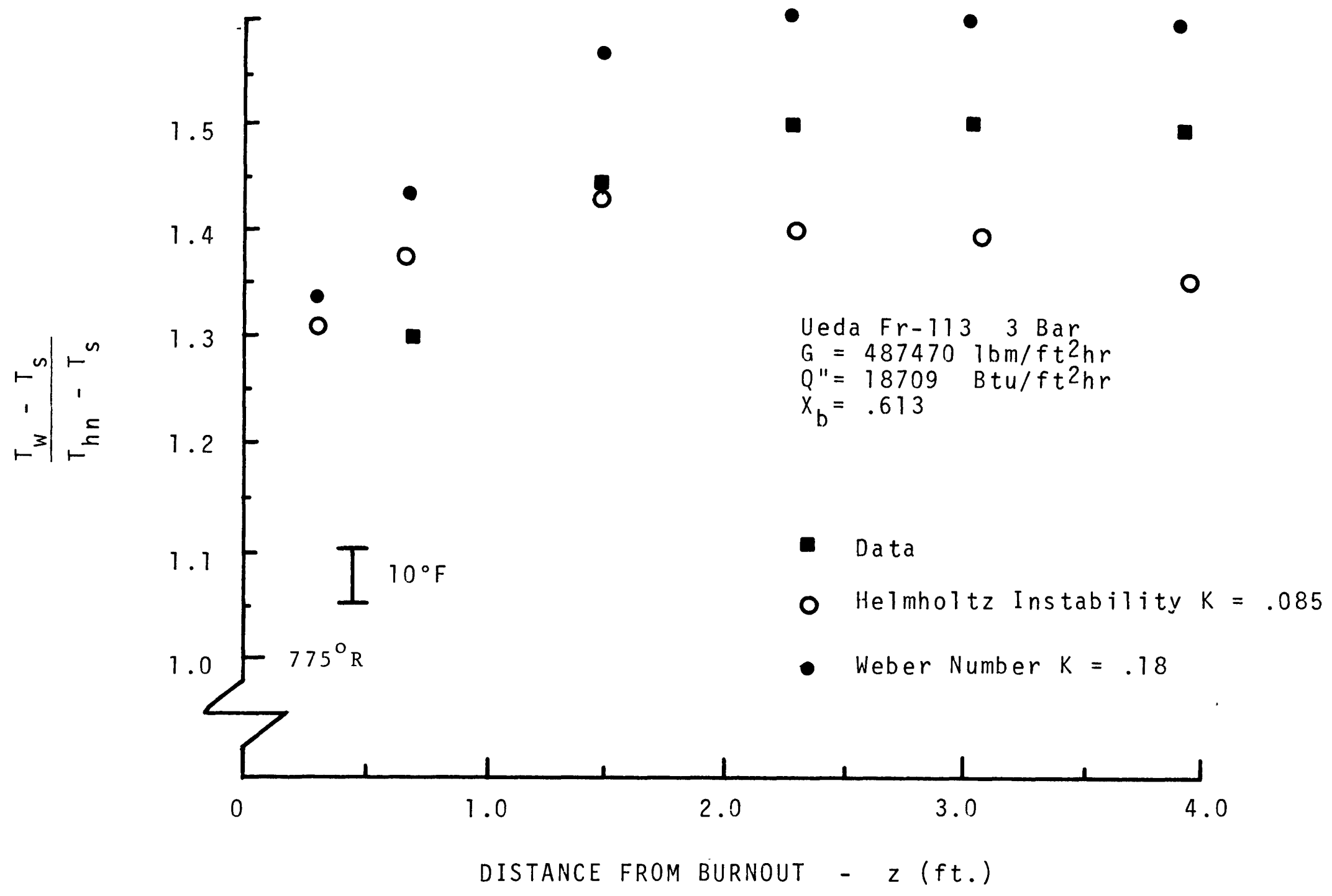

FIGURE 4-19 


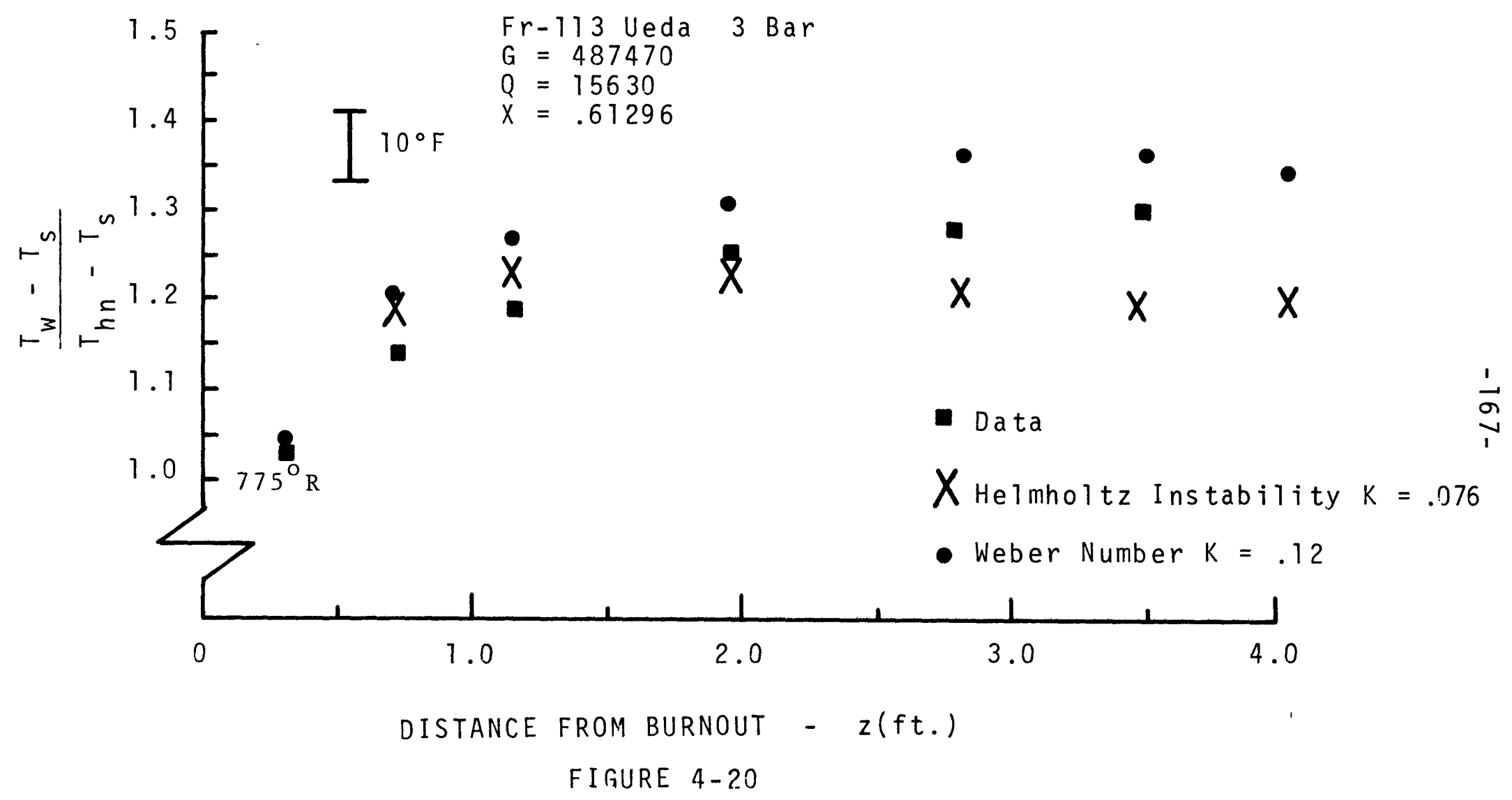




\section{$-168-$}

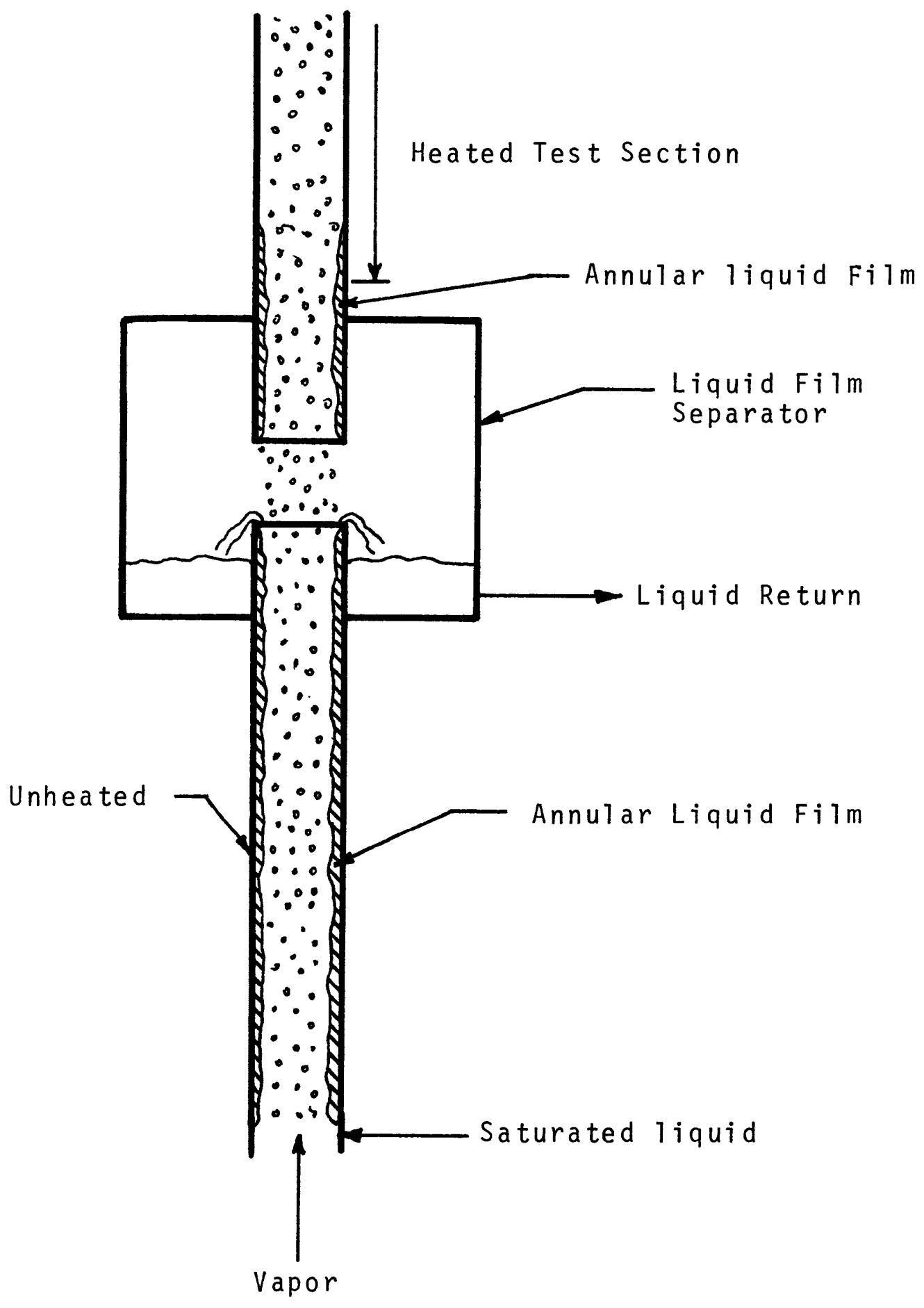

FIGURE 4-21 Ueda's Apparatus 
temperature curves. The upper points are predicted using the film slip Weber number drop size while the lower set of points are the predicted wall temperatures using a Helmholtz drop size. As is shown, the data lies between these curves in both Figures 4-19 and 4-20 as would be expected.

Helmholtz drop sizes are smaller than critical Weber number drop sizes, and more equilibrium is predicted to exist in the flow (lower K) if the Helmholtz drop size is used.

\section{3b Effect of a Variation in the Constant $K$}

Equation (4-40) shows directly how various property values and flow conditions effect the constant $k$. The value of $k$ is an indication of the amount of non-equilibrium within the flow, which in turn effects the wall temperature. Figure 4-22 shows how a variation in $K$ alters predicted wall temperatures. $K=0.4$ is the value predicted using the procedure in section 4-2. A higher $K$ value predicts higher wall temperatures since more non-equilibrium in the flow means both higher bulk vapor temperatures and lower vapor velocities (the local value of quality decreases as non equilibrium increases). 


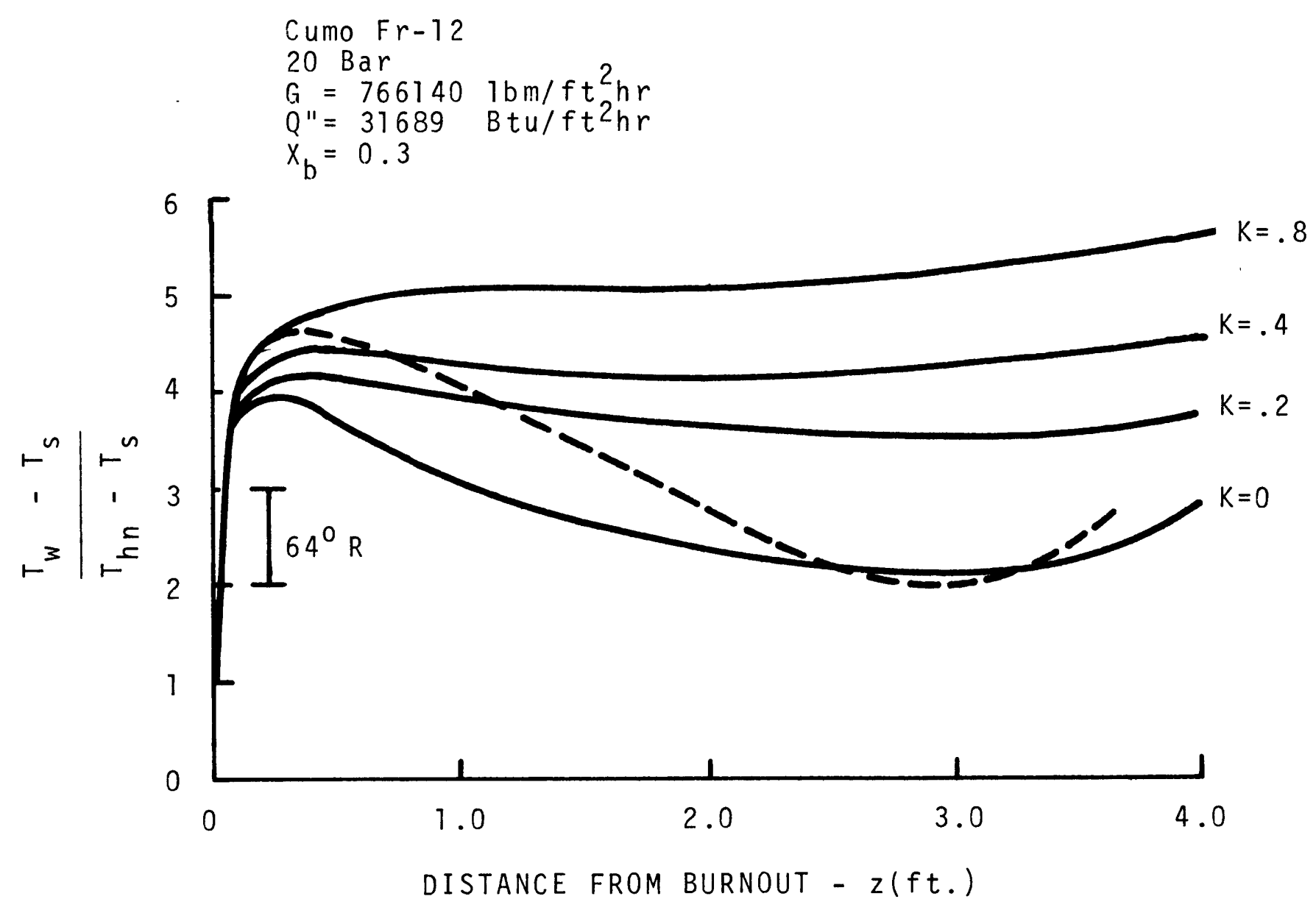

FIGURE 4-22 Variation in the Constant $K$ 
Figure 4-22 is also an indication of how an error in calculating the value of $K$ (i.e. how well burnout canditions are known)will affect wall temperature predictions. In this case a $25 \%$ variation in $K$ from the predicted value of 0.4 would result in a variation in wall temperature of about $25^{\circ} \mathrm{F}$.

The conditions chosen to illustrate the effect of $K$ were those of one of the test runs of Cumo for Freon-12. The dotted curve of Figure 4-22 represents the Cumo data of Figure 4-17 and falls below the prediction for $K=0$ or equilibrium vapor conditions, thus casting serious doubt about the validity of the data.

\subsection{Discussion of the Local Conditions Solution}

The local conditions solution appears rather complicated as it must be in order to predict a complicated heat transfer phenomenon. The scheme does afford the advantage of explicitly showing the effect of various parameters on non-equilibrium in the flow. It also allows calculation of wall temperatures without the aid of a computer by establishing local conditions using only known values, $X_{b}$, $G, Q ", D_{T}$, and property values. All local conditions can be established once a value for the constant $K$ is 
determined. The solution uses no empirical constant, other than those contained in accepted single phase correlations.

The calculation scheme can be divided into three separate sections.

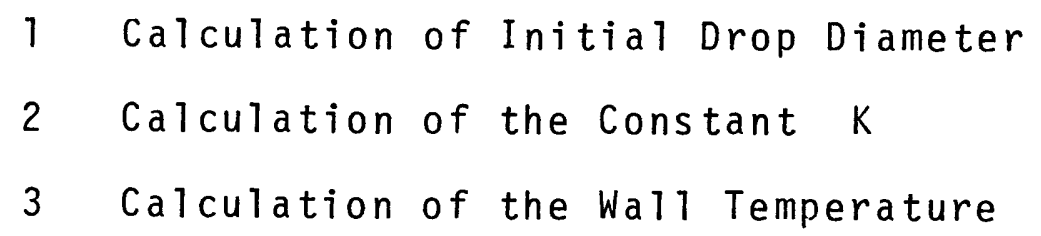

Calculation of the initial drop diameter is the most complex of the three steps. Initial drop sizes must be calculated which account for drop breakup after burnout, as well as accounting the flow history previous to burnout.

The constant $K$ consists only of property values, the initial drop size, and local flow conditions at the burnout point. Calculation of $K$ involves six separate steps, however, an algebraic expression for $K$ can be obtained for many flow situations. This equation explicitly shows the effect of variables on the magnitude of $k$.

Wall temperature calculations begin by using graphs of $x$ vs. $\left(x_{e q}-x_{b}\right)$ to determine non-equilibrium in the flow. Local vapor temperatures can then be determined. The remainder of the wall temperature calculation is simi- 
lar to that which must be used for turbulent single phase forced convection.

Comparison of the local conditions model with wall temperature data of several investigators shows that the scheme is a reasonable approach to wall temperature calculation. Wall temperatures predicted by the local conditions method agree well with wall temperatures predicted with the numerical method. 
CHAPTER $V$

\section{SUMMARY}

Dispersed flow heat transfer consists of several individual heat transfer mechanisms. An accurate analysis of dispersed flow must include at least the most important of these. Interactions between the drops, the vapor, and the heated wall, influence both the wall heat transfer and the non-equilibrium existing in the flow. These in turn determine the tube wall temperatures.

Specifically, this study investigates dispersed flow heat transfer for vertical upflow, constant heat flux conditions. Droplet formation and breakup is modelled by considering both the flow history previous to burnout, and the flow regime preceeding dispersed flow formation. A mass averaged drop diameter is calculated using known flow conditions and burnout quality. Drop diameters calculated by this method are used in both a numerical and a local conditions solution.

A numerical model based on two phase transport equations is used to predict heat transfer downstream of burnout. Mechanisms in the model include wall-to-drop, vaporto-drop, wal1-to-vapor, and conduction heat transfer. 
Existing correlations are used for the individual mechanisms, eliminating the need for empirical constants.

A local conditions solution based on the same transport equations is possible using conclusions from the numerical model. This solution identifies one non-dimensional group which alone determines the non-equilibrium present in the flow. Wall temperatures may then be hand calculated without using numerical techniques.

Both the numerical and local conditions solution were compared to world data with acceptable results. Nitrogen, Freon, and water data from five investigators and various flow conditions were compared. Wall temperatures predicted by the numerical and local conditions solution agree to within about $5 \%$ for all conditions tested.

CONCLUSIONS

1. A numerical model which includes four individual heat transfer mechanisms predicts wall temperatures which agree well with wall temperature data for several fluids.

2. The wall-to-droplet heat transfer mechanism was found to have little effect on predicted wall temperatures except very near the burnout point. 
3. Axial conduction within the tube has very little effect on wall temperatures when the tube material has thermal properties similar to inconel.

4. A single non-dimensional grouping indicates the degree of non-equilibrium within the flow.

5. A local conditions solution based on this nondimensional grouping can be used to hand calculate tube wall temperatures under dispersed flow conditions.

\section{RECOMMENDATIONS}

Several areas which would benefit from further investigation can now be Adentified.

Information about the area immediately beyond burnout is still lacking. Both experimental and theoretical investigations are needed for both annular and inverted annular burnout to determine the effect of the droplets on boundary layer growth and the effect of the liquid core in inverted annular flow.

Dispersed flow droplet sizes should be characterized more completely. An experimental investigation is needed to determine the actual drop size distributions present in heated flows. 
Deposition and entrainment data is also needed in heated annular flows in order to determine the origin of drops existing at burnout. 


\section{FREE STREAM SLIP DETERMINATION}

The assumption $x_{e q} \frac{d \frac{1}{s \alpha}}{d z}<\frac{1 d x_{e q}}{s \alpha d z}$ leads to an equation for free stream slip, Equation (3-5). This equation can be compared to numerically calculated values of slip without this assumption where the vapor is accelerating at at rate determined by the local equilibrium quality. Figure Al-1 compares values of slip calculated from Equation (3-5) and numerically calculated slip ratios under the same conditions. Values of liquid density, heat flux, and droplet diameter were varied to cover a range of flow conditions. All other properties were those of saturated nitrogen at 17 psi.

Equation (3-5) predicts values of slip which are close to the numerically calfulated slip ratios. Thus, the assumption that $x_{e q} \frac{d \frac{1}{s \alpha}}{d z}<\frac{1}{s \alpha} \frac{d x_{e q}}{d z}$ is reasonable when the flow is in equilibrium, and the droplet size remains constant. These are the conditions which are present at burnout, and Equation (3-5) should be a good approximation to the actual free stream slip at burnout.

At points downstream from burnout, the equilibrium 


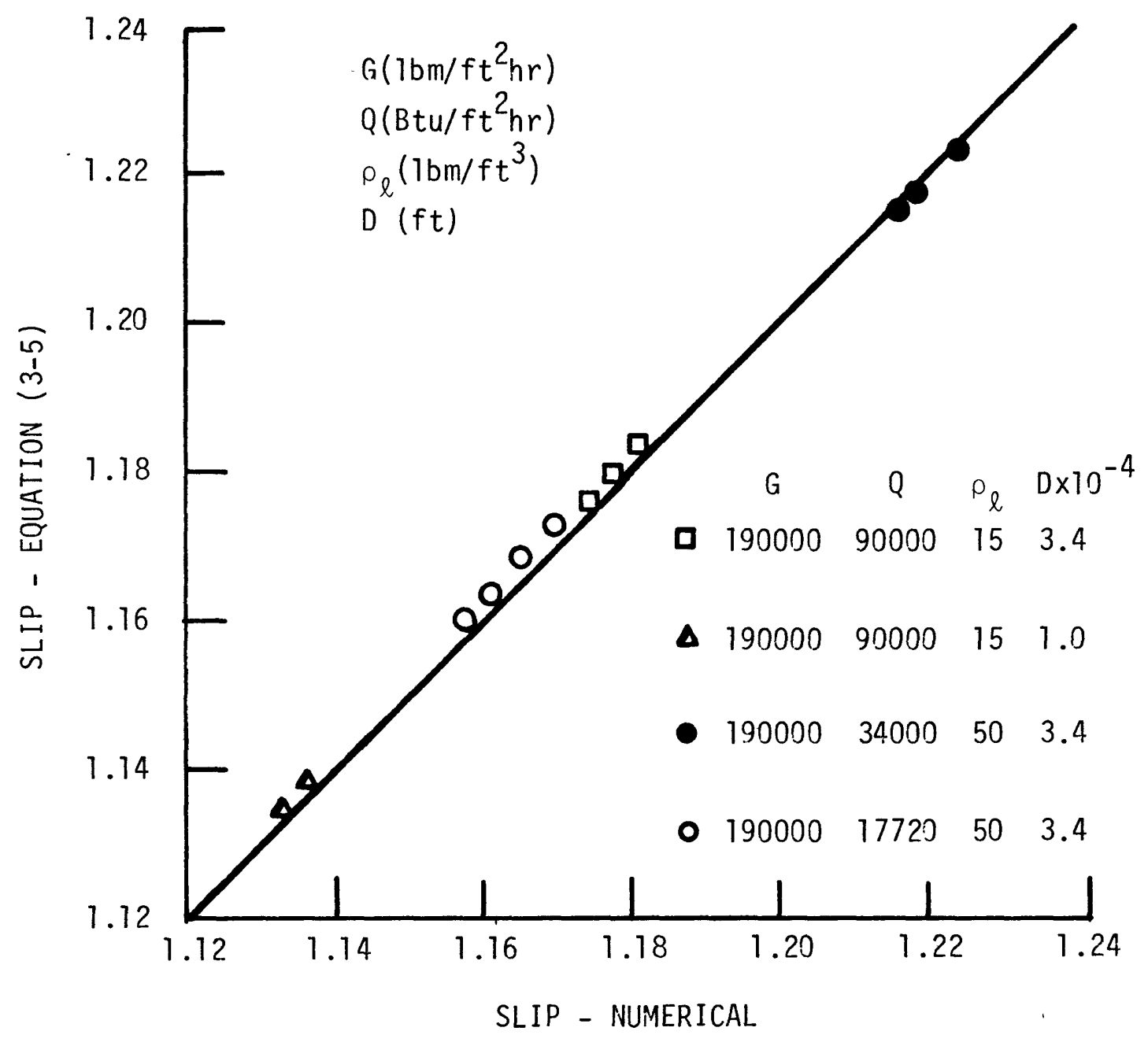

FIGURE A1-1 Free Stream S1 ip Comparison 
condition does not hold, and droplet diameters are decreasing with distance. The local conditions model assumed that $\frac{d X}{d z} \simeq \frac{d x_{e q}}{d z}$ locally, along with the assumption

$$
x_{\text {eq }} \frac{d \frac{1}{s \alpha}}{d z}<\frac{1}{s \alpha} \frac{d x_{e q}}{d z}
$$

in order to arrive at a slip equation, (4-6) which is valid at any point in the tube.

These assumptions can also be examined by comparing the local slip ratio at any point in the tube calculated by equation (4-6) to that predicted by the numerical heat transfer model described in Chapter III. Figure Al-2 shows slip ratios calculated by each method for several flow conditions.

Equation (4-6) predicts values of slip which agree well with those calculated numerically and it can be assumed valid at any point in the tube. 


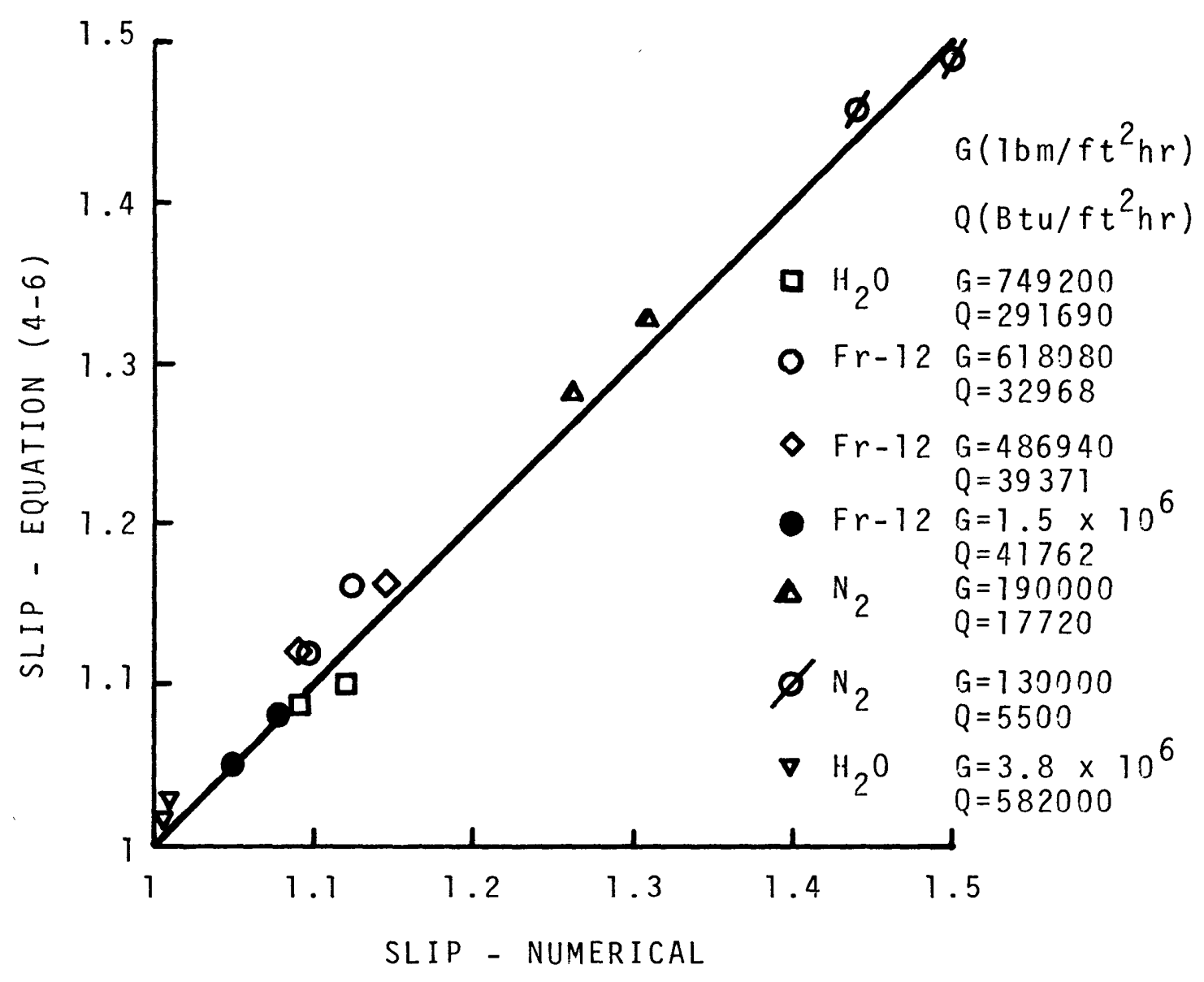

FIGURE AI-2 Free Stream S1 ip Comparison 


\section{APPENDIX A2}

\section{RADIATION}

Radiation from the hot wall to the droplets helps decrease wall temperatures as well as evaporate the drops. The radiation heat transfer from the wall to the droplets can be found by using a gray body factor between the wall and drops, $F_{W D}$

$$
q_{R}^{\prime \prime}=F_{W D} \psi\left(T_{W}^{4}-T_{S}^{4}\right)
$$

assuming the drops are at the saturation temperature. $\psi$ is the Stefan-Boltzman constant. Evaluation of $F_{W D}$ was presented by Sun et al [48] for a droplet cloud, assuming the droplets were in the scattering regime. In dispersed flow, droplet diameters are larger than the characteristic radiative wavelength and this assumption is valid. From Sun's analysis

$$
F_{W D}=1 /\left(\frac{1}{\gamma_{D}}+\frac{1}{\gamma_{W}}-1\right)
$$


where $\gamma_{W}$ is the wall emissivity $(\approx .76$ for inconel) and $\gamma_{D}$ is the emissivity of the droplet cloud given by

$$
\gamma_{D}=1-e^{-1.11(1-\alpha)} \frac{D_{T}}{D}
$$

Table A2-1 compares the radiative heat flux, $q_{R} "$ to the total heat flux, and also shows the magnitude of the radiation term in Ross' shielding function for droplet heat transfer coefficients. Wall temperatures are the average of the maximum and minimum predicted wall temperatures for any flow conditions. Property values are based on conditions existing at the mid point of the tube.

\begin{tabular}{|c|c|c|}
\hline Fluid ${ }_{0}^{2}\left(1 \mathrm{bm} / \mathrm{ft}^{2} \mathrm{hr}\right)$ & $\underline{q_{R} "}$ & $q_{R}^{\prime \prime}$ \\
\hline & Q" & in $h_{f g}$ \\
\hline $\begin{array}{l}\mathrm{Fr}-12 \\
18709\end{array}$ & .015 & .029 \\
\hline $\begin{array}{l}\mathrm{H}_{2} \mathrm{O} \\
291690\end{array}$ & .017 & .023 \\
\hline $\begin{array}{l}\mathrm{Fr}-12 \\
39371\end{array}$ & .013 & .036 \\
\hline $\begin{array}{l}\mathrm{N}_{2} \\
17720\end{array}$ & .016 & .017 \\
\hline
\end{tabular}

Table A2-1 Radiative Heat Exchange 


\section{EFFECT OF DROPS ON BOUNDARY LAYER GROWTH}

In the area immediately beyond the burnout point, a thermal boundary layer is growing in the flow. In all calculations presented in this thesis, a vapor thermal entrance length based on Kays numerical computations was used. However, the presence of drops in the boundary layer can slow the boundary layer growth by acting as a sink in the flow, thus increasing the entrance length.

An analysis of this phenomenon has been presented by Rane and Yao [44]. They solved the two dimensional heat transfer equations for tube flow with a sink present. A fully developed velocity profile was assumed to be present at the beginning of the heated length. Although the droplets were allowed to evaporate, altering the vapor velocity and void fraction and therefore the droplet number density, they assumed that droplet diameters remained constant in the flow.

A conventional integral analysis can also be modified to account for the presence of a sink in the flow. Figure A3-1 shows a portion of the developing boundary layer. Droplets and vapor are both flowing from left to right in the figure. A temperature profile exists within 
BOUNDARY LAYER GROWTH WITH A SINK

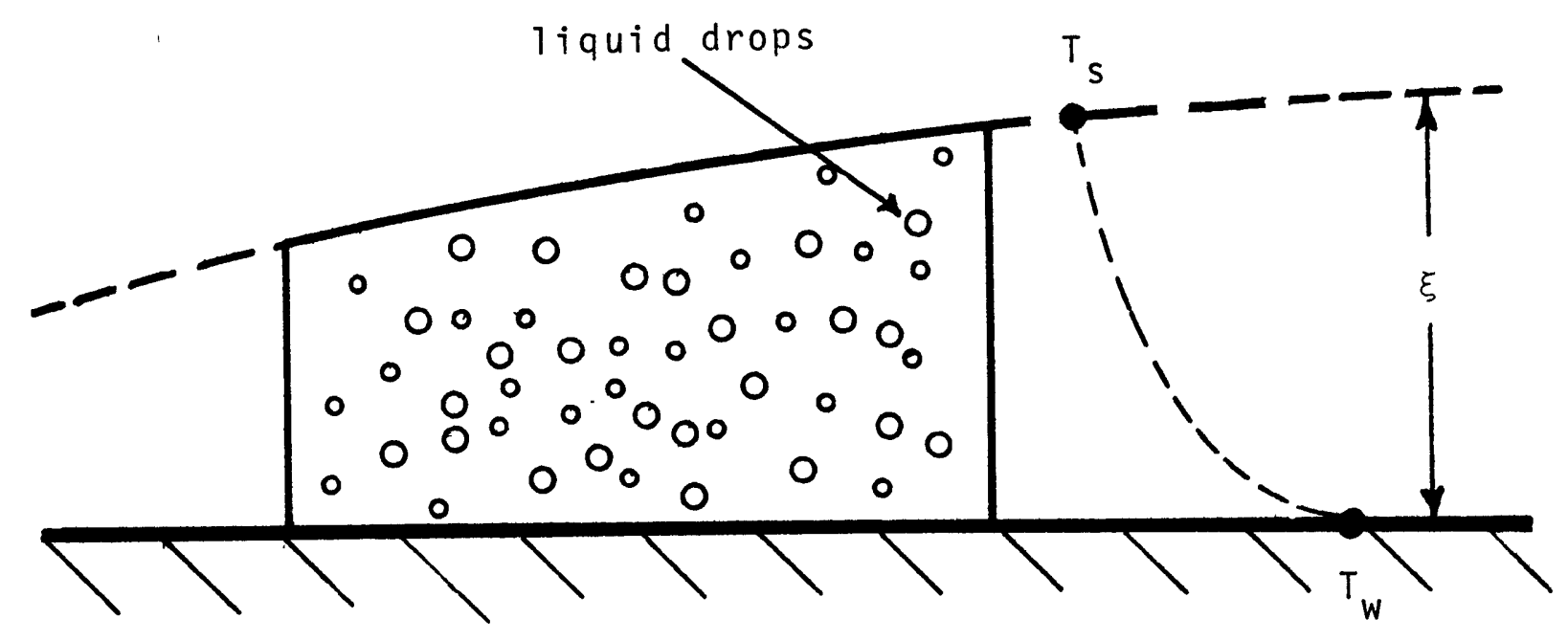

FIGURE A3-1 
the boundary layer, with the edge of the layer at the saturation temperature, $T_{S}$ and the wall at temperature $T_{W}$. Droplets in the flow are at the saturation temperature, and evaporate due to the difference in local vapor and liquid temperatures. An energy balance on the boundary layer can be written.

(Energy into vapor of the boundary layer) = (energy from the wall) - (energy to the droplets)

If the drop- to -wall heat transfer contribution is ignored, the energy absorbed by the sink is due to the difference in temperature between the vapor and liquid. The heat transfer rate to the drops per unit volume can be written as the sum of the energy needed to evaporate the drops and the energy needed to heat the resulting vapor to free stream conditions.

$$
n \pi D k_{v} N u_{D}\left(T_{v}-T_{s}\right)+n \pi D k_{v} N u_{D} C_{p}\left(T_{v}-T_{s}\right) / h_{f g}
$$

where $n$ is the number of drops per unit volume, and $N u_{D}$ is the droplet Nusselt number defined by Equation (3-31), Equation A3-1 can now be written in terms of $\mathrm{Nu}_{0}$, Equation $(3-32)$. 


$$
n \pi D k_{v} N u_{0}\left(T_{v}-T_{s}\right)
$$

The energy into the fluid from the wall is determined by the temperature gradient at the wall.

$$
\left.-k_{v} \frac{d T}{d y}\right)_{0}
$$

The energy used to heat the vapor in the boundary 1ayer is

$$
\rho_{v} C_{p} v_{v}\left(T-T_{s}\right)
$$

The boundary layer integral equation can thus be written using $(A 3-2),(A 3-3)$ and $(A 3-4)$

$$
\begin{gathered}
\left.\frac{d}{d z} \rho_{v} C_{p} \alpha \int_{0}^{\xi} V(y)\left(T-T_{s}\right) d A=-k_{v} \frac{d T}{d y}\right)_{0} P- \\
N u_{0} k_{v} n \pi D \int_{0}^{\xi}\left(T-T_{s}\right) d A
\end{gathered}
$$

where $P$ is the heated perimeter and $A$ is the flow area of the boundary layer.

For most cases of practical interest, the entrance length is quite short compared to the length over which the drops evaporate, and drop diameter and droplet number 
density can be considered constant. At the burnout point, the liquid film thickness is much smaller than the tube diameter and the vapor velocity profile can be treated as fully developed. Appropriate velocity and temperature profiles must be chosen to correspond to the flow regime present.

The simplest flow regime to analyze is laminar flow. Hence, it will be used to demonstrate the integral technique. The velocity profile in fully developed laminar pipe flow can be assumed to be parabolic

$$
\frac{v}{v_{v}}=8\left(1-\frac{y}{D_{T}}\right) \frac{y}{D_{T}}
$$

where $u$ is the local velocity in the $z$ direction and $y$ is the distance from the tube wall.

The temperature profile in the boundary layer can be assumed to have the following form.

$$
\frac{T_{v}-T_{s}}{T_{w}-T_{s}}=\left(1-\frac{y}{\xi}\right)^{2}
$$

where $\xi$ is the boundary layer thickness. For a constant wa11 temperature, Equations (A3-6) and (A3-7) can be substituted into Equation ( $A 3-5)$, assuming $d A \approx \pi D_{T} d y$ and the void fraction, $\alpha$ is near 1 


$$
\begin{gathered}
8 \frac{d}{d z}\left[\rho_{v} C_{p} v_{v} \int_{0}^{\xi}\left(1-\frac{y}{D_{T}}\right) \frac{y}{D_{T}}\left(1-\frac{y}{\xi}\right)^{2}\left(T_{w}-T_{s}\right) d y\right]=\frac{2 k_{v}}{\xi}\left(T_{w}-T_{s}\right) \\
-N u_{0} k_{v} n \pi D \int_{0}^{\xi}\left(1-\frac{y}{\xi}\right)^{2}\left(T_{w}-T_{S}\right) d y \quad A 3-8
\end{gathered}
$$

This equation can now be integrated for $\left(T_{W}-T_{S}\right)$ constant.

$$
\begin{gathered}
z^{+}=\frac{2}{M}\left\{\frac{1}{3} \frac{1}{\sqrt{M}} \ln \left[\frac{1+\sqrt{M} \xi^{+}}{1-\sqrt{M} \xi^{+}}\right]-\xi^{+}\left(\frac{2}{3}-\frac{1}{5} \xi^{+}\right)\right. \\
\left.-\frac{1}{5} \frac{1}{M} \ln \left(1-M \xi^{+2}\right)\right\}
\end{gathered}
$$

where

$$
\begin{aligned}
M & =\frac{1}{6} N u_{D} \cap \pi D D_{T}{ }^{2} \\
\xi^{+} & =\frac{\xi}{D_{T}}
\end{aligned}
$$

and

$$
z^{+}=\frac{2 z}{D_{T} \operatorname{Re} P r}
$$

The wall Nusselt number based on the saturation temperature $\mathrm{Nu}$ ws can be calculated from the assumed temperature profile. 


$$
\left.N u_{w s}=-D_{T} \frac{d T}{d y}\right)_{0} \frac{1}{\left(T_{w}-T_{s}\right)}=\frac{2}{\xi^{+}}
$$

Nusselt numbers as a function of $z^{+}$and various values of $M$ are plotted in Figure A3-2 along with values calculated by Rane. The Nusselt numbers become constant in Equation (A3-9) once the sink becomeslarge enough to absorb all energy entering the boundary layer. Rane includes the effect of evaporation on the vapor velocity and droplet number density. Because the number density decreases down the tube, his calculated Nusselt numbers decrease with distance. Also, as the droplet loading or sink term gets large, vapor velocities increase more rapidly as more vapor is generated due to evaporation. This would explain the difference in Nusselt numbers calculated by Rane and Equation (A3-9) at higher droplet loadings. The integral technique also assumes that the shape of the temperature profile is unaffected by the presence of droplets, and only the boundary layer growth rate decreases as droplet loading increases.

Equation (A3-9) gives the same general dependence of Nusselt number on droplet loading as does the more complex numerical solution of Rane. It could therefore be used to estimate the effect of droplets on vapor 


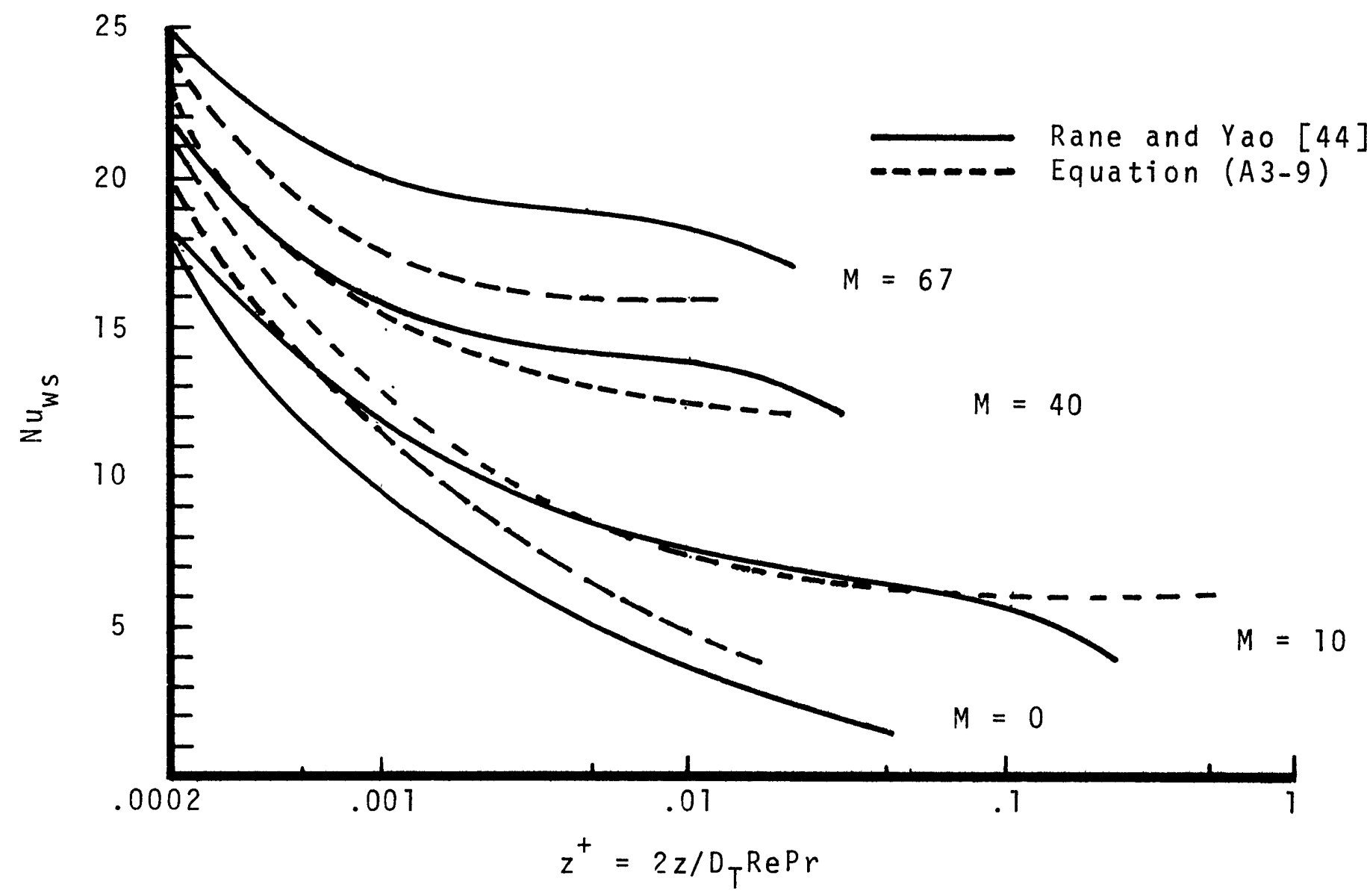

FIGURE A3-2 Equation (A3-9) and Rane's snlution for Eoundary Layer Growth with a Sink Present 
thermal entrance lengths.

The general integral technique can also be applied to other geometries and flow regimes. For example, turbulent flow could be investigated using appropriate temperature and velocity profiles inserted in the boundary Tayer Equation (A3-5). 


\section{APPENDIX A4}

\section{SAMPLE CALCULATION}

As an example we use one of Groeneveld's Freon-12 runs with the following flow conditions:

$$
\begin{aligned}
& G=486,940 \frac{1 \mathrm{bm}}{\mathrm{ft}^{2} \mathrm{hr}} \\
& Q=39371 \frac{\mathrm{Btu}}{\mathrm{ft}^{2} \mathrm{hr}} \\
& X_{b}=.649 \\
& D_{T}=.0256 \mathrm{ft} \\
& \text { Pressure }=160 \mathrm{psia}
\end{aligned}
$$

Calculate the constants (this example is in English units)

$$
\tau=\frac{(4)\left(4.45 \times 10^{-4}\right)\left(4.17 \times 10^{8}\right)}{(486940)^{2}(.0256)}=1.22 \times 10^{-4}
$$

$$
\begin{aligned}
& A c=\frac{39371}{(486940)(53.4)}=1.5 \times 10^{-3} \\
& G r=\frac{(4)(77.0-4)(.0256)\left(4.7 \times 10^{8}\right)}{(486940)^{2}}=1.315 \times 10^{-2}
\end{aligned}
$$




$$
\begin{aligned}
\frac{D_{b}}{D_{T}} & =\left(\frac{3\left(1.22 \times 10^{-4}\right)(6.5)(.4)}{16\left(1.5 \times 10^{-3}\right)\left(\frac{77}{4}\right)(.649)+4\left(1.315 \times 10^{-2}\right)}\right)^{1 / 2} \\
& =5.19 \times 10^{-2}
\end{aligned}
$$

Check the magnitude of the gravity term assuming the void fraction equals 1 .

$$
\frac{4}{3}\left(1.315 \times 10^{-2}\right) \frac{\left(5.19 \times 10^{-2}\right)}{(.649)^{2}(.4)}=5.4 \times 10^{-3} \ll 1
$$

we can therefore use Equation (4-7a) for the free stream slip calculation

$$
\begin{aligned}
& S=1+\left[\frac{16(77)\left(1.5 \times 10^{-3}\right)\left(5.19 \times 10^{-2}\right)}{3(4)(.649)(.4)}\right]^{1 / 2} \\
& S=1.18 \\
& \alpha=\frac{1}{(4)(1.18)(1-.649)}+1 \\
& (77)(.649)
\end{aligned}
$$

$s \alpha=1.14$ which is less than 1.7 and this method of calculating the drop diameter is valid. The film slip is given by Equation (2-4) 


$$
S_{f}=\left(\frac{77}{4}\right)^{0.205}\left[\frac{(486940)(.0256)}{(.445)}\right]^{-.016}=1.56
$$

Calculation of $x_{a}$ and $\bar{D}$

$$
\begin{aligned}
& {\left[\frac{4}{77}+\left(\frac{1}{1.56}-\frac{4}{77}\right) x_{a}\right] /\left[\frac{4}{77}+\left(\frac{1}{1.17}-\frac{4}{77}\right) .649\right]} \\
& =\frac{1.17-1}{1.56-1} \\
& x_{A}=.21
\end{aligned}
$$

Calculate

$$
\begin{gathered}
\frac{\rho_{\ell}}{\rho_{v}} \frac{1}{S_{f}}-1=\frac{77}{(4)(1.56)}-1=11.34 \\
\frac{\bar{D}}{D_{T}}=\frac{1}{.649-.1}\left\{5.19 \times 10^{-2}(.21-.1)+\left(\frac{77}{4}\right)^{2} \frac{\left(1.22 \times 10^{-4}\right)(6.5)}{(1.58-1)^{2} 11.34} \cdot\right. \\
\left.\left[\frac{1}{1+.21(11.34)}-\frac{1}{1+.649(11.34)}\right]\right\} \\
\frac{\bar{D}}{D_{T}}=3.51 \times 10^{-2}
\end{gathered}
$$

Calculation of $X_{T A}$

$$
x_{T A}=\frac{3}{10} \frac{1.315 \times 10^{-2}}{1.5 \times 10^{-3}} \frac{4}{77}=.55
$$


$X_{B 0}>X_{T A} \therefore$ no drop breakup will occur, and $D_{0}=\bar{D}$

Calculation of $\mathrm{K}$

$$
\begin{aligned}
& K=.58\left(3.51 \times 10^{-2}\right)^{1.25}(1-.649)^{-5 / 12}\left(1.5 \times 10^{-3}\right)^{.75} \frac{77}{4} .75 \\
& {\left[\frac{(486940)(.0256)}{.031}\right]^{1 / 2}(.97)^{2 / 3}\left[\frac{(4)(1-.649)}{(77)(.649)}+\right.} \\
& \left.\left\{1+3.65\left[\left(1.5 \times 10^{-3}\right) \frac{77}{4} \frac{3.51 \times 10^{-2}}{.649}\right]^{1 / 2}\right]^{-\frac{1}{2}}\right]^{\frac{1}{2}}(4-40) \\
& K=.58
\end{aligned}
$$

This value of $k$ now allows determination of all local conditions using the graphs of $K$ (Figures 4-3 to 4-11). The burnout quality for this data is .649 , and the curves must be interpolated. One method of interpolation which gives satisfactory results is to use the graph for burnout quality closest to the burnout quality of the data, and simply "stretch" or "compress" the curves. This can be accomplished by fixing the upper bound of $\left(x_{e q}-x\right)$ and $x$ and linearly scaling the absissa and ordinate.

The absissa can be scaled as follows: 


$$
\frac{x_{\text {upper }}-x_{b}{ }^{\prime} \text { actual }}{x_{\text {upper }}-x_{b}{ }_{\text {graph }}}=\frac{x_{\text {upper }}-x_{e q}{ }^{\prime} \text { actual }}{x_{\text {upper }}-x_{\text {eq }}{ }^{\prime} \text { graph }}
$$

The ordinate can be scaled similarly

$$
\frac{\left.1-x_{b}\right)_{\text {actual }}}{\left.1-x_{b}\right)_{\text {graph }}}=\frac{1-x)_{\text {actual }}}{1-x)_{\text {graph }}}
$$

For our case, using the graph $x_{b}=0.6$, this becomes

$$
\begin{aligned}
& \frac{2.951}{3.0}=\frac{3.6-x_{e q}{ }^{\prime} \text { actual }}{\left.3.6-x_{e q}\right)_{\text {graph }}} \\
& 1.02 x_{\text {eq }}{ }^{\prime} \text { actual }_{\text {actua }}-.06=x_{\text {eq }}{ }^{\prime} \text { graph }
\end{aligned}
$$

and for the ordinate,

$$
\text { X) } \text { actual }=.123+.878 x)_{\text {graph }}
$$

Equilibrium quality $x_{e q}$ for any point in the tube is determined using Equation (4-21)

$$
x_{e q}=\frac{4(39371)}{(486940)(53.4)(.0256)} z+.649
$$

for example at a distance of $z=.5 \mathrm{ft}$ 


$$
x_{\text {eq }}=.77
$$

and $\left.x_{\text {eq }}\right)_{\text {graph }}=.714$ or $\left(x_{e q}-x_{b}\right)_{g r a p h}=.11$

using the graph of $x_{0}=.6$ for $k=.58$,

$$
x)_{\text {graph }}=.615
$$

and

$$
x)_{\text {actual }}=.66
$$

The vapor temperature can now be calculated using Equation (4-27)

$$
\begin{aligned}
& T_{v}-T_{s}=\frac{53.4}{.19}\left(\frac{.76}{.66}-1\right) \\
& T_{v}-T_{s}=43
\end{aligned}
$$

The void fraction calculated for $D_{b}$ was shown to be 0.97 . Since the drop diameter at $x=.67$ will be smaller than $D_{b}$ a good assumption for this case is to assume $\alpha \approx 1$.

The wall-to-vapor Nusselt number can now be calculated using local vapor properties. 


$$
\begin{gathered}
N u_{w}=\frac{h_{w} D}{k_{v}}=.023\left[\frac{(486940)(.66)(.0256)}{.038}\right]^{.8}(.98) \cdot 4\left[1+\frac{.8(.0256)}{.5}\right] \\
N u_{w}=440
\end{gathered}
$$

The wall temperature is then given by Equation (4-39)

$$
\begin{aligned}
& T_{W}=43+573.4+\frac{39371(.0256)}{440(.007)} \\
& T_{W}=944^{\circ}{ }^{\circ}
\end{aligned}
$$

using the same value for $K$, the same procedure can be used to calculate wall temperatures at any point in the tube.

$\begin{array}{cccccc}z(f t) & \left(x_{e q}-x_{b}\right) & x & T_{v}-T_{S}\left({ }^{0} R\right) & N_{W} & T_{W}\left({ }^{0} R\right) \\ .5 & .11 & .66 & 43 & 440 & 944 \\ 1.0 & .24 & .692 & 83 & 420 & 977 \\ 1.5 & .36 & .71 & 124 & 398 & 1000 \\ 2.0 & .49 & .764 & 146 & 398 & 1001 \\ 3.0 & .73 & .834 & 194 & 414 & 1030 \\ 4.0 & .97 & .892 & 242 & 415 & 1036\end{array}$

Table A4-1 CALCULATED WALL TEMPERATURES

This profile is plotted in Figure 4-16. 


\section{APPENDIX A5}

\section{DROPLET ENTRAINMENT AND DEPOSITION}

Some droplets entrained in the annular portion of the flow regime deposit back on the liquid film as shown in Figure A5-1. A small portion of the entrained droplets, which were entrained at one section, A can be followed as they travel downstream. These droplets are characterized by a diameter corresponding to the quality at which they were formed, $x_{A}$, using Equation (2-10). As the single sized droplet group moves downstream, some of the droplets deposit back on the wall due to vapor fluctuations in the transverse direction. The number of droplets in the group, and therefore the mass within the group decreases as it travels downstream. Because the quality varies down the tube, the characteristic drop diameter changes. Thus, many small groups of droplets, each characterized by a single droplet size are present in the flow at any point. In order to determine an average drop size at burnout, we must know the mass remaining in each of these groups at burnout. To do this, droplet entrainment and deposition must be known.

Very little entrainment or deposition data is avail- 


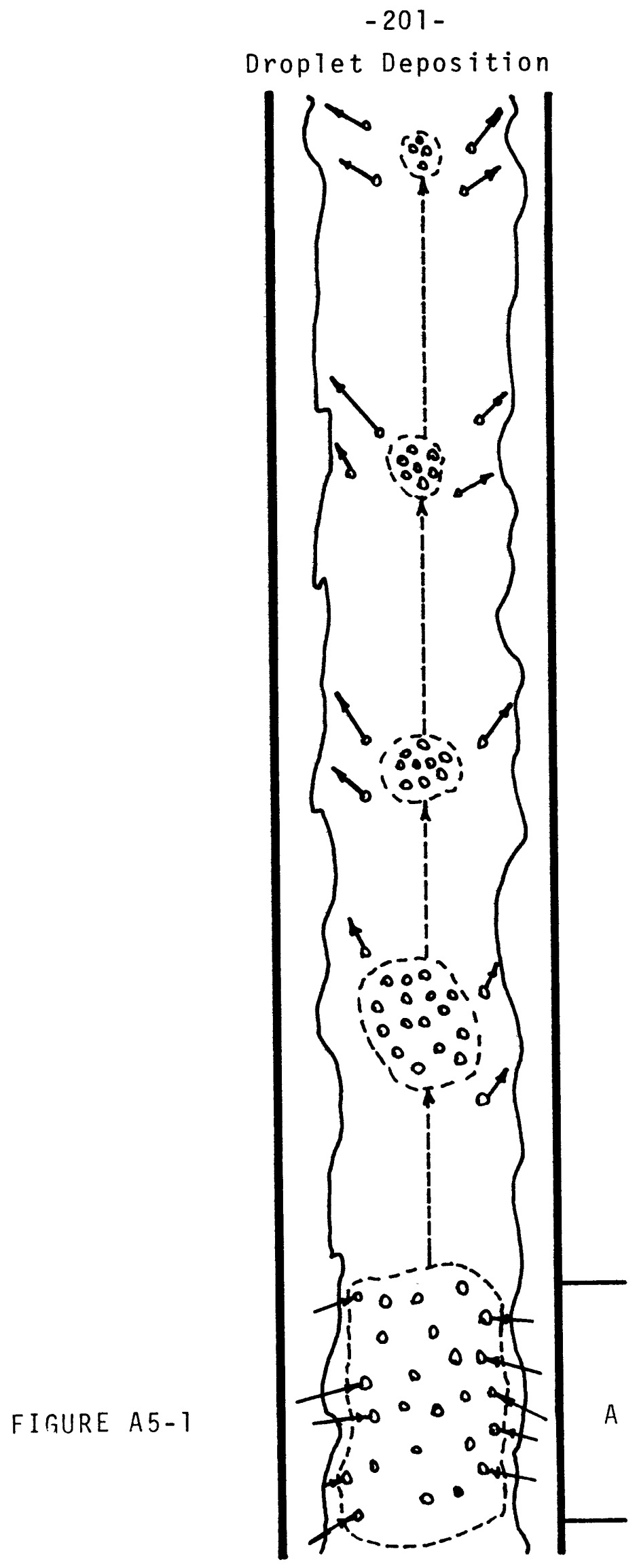


able for heated annular flows. Hewitt [35] presented total entrainment data for two flow conditions. One of these curves is shown in Figure A5-2. Droplet deposition rates must also be known as well as total entrainment rates in order to calculate the mass cumulative distribution, E at burnout. Whalley et al [49] calculated droplet deposition rates for annular flows assuming that the deposition rate is proportional to the mass concentration of droplets in the core

$$
\Gamma^{\prime \prime}=\kappa C
$$

where $\kappa$ is the mass transfer coefficient, and $C$ is the concentration of droplets in the core calculated on the basis of a homogeneous flow.

We have used this method of calculation to determine deposition rates for the data of Figure A5-2.

A deposition coefficient of $5904 \mathrm{ft} / \mathrm{hr}$ was chosen by extrapolating the value of deposition coefficients recommended by Whalley to the appropriate pressure. The history of several droplet groups is shown in Figure A5-3. The mass entrained, e over the quality $\Delta X=0.1$ is shown for six points in the tube. Each solid curve shows the mass remaining in each droplet group. The sum of the curves 
$-203-$

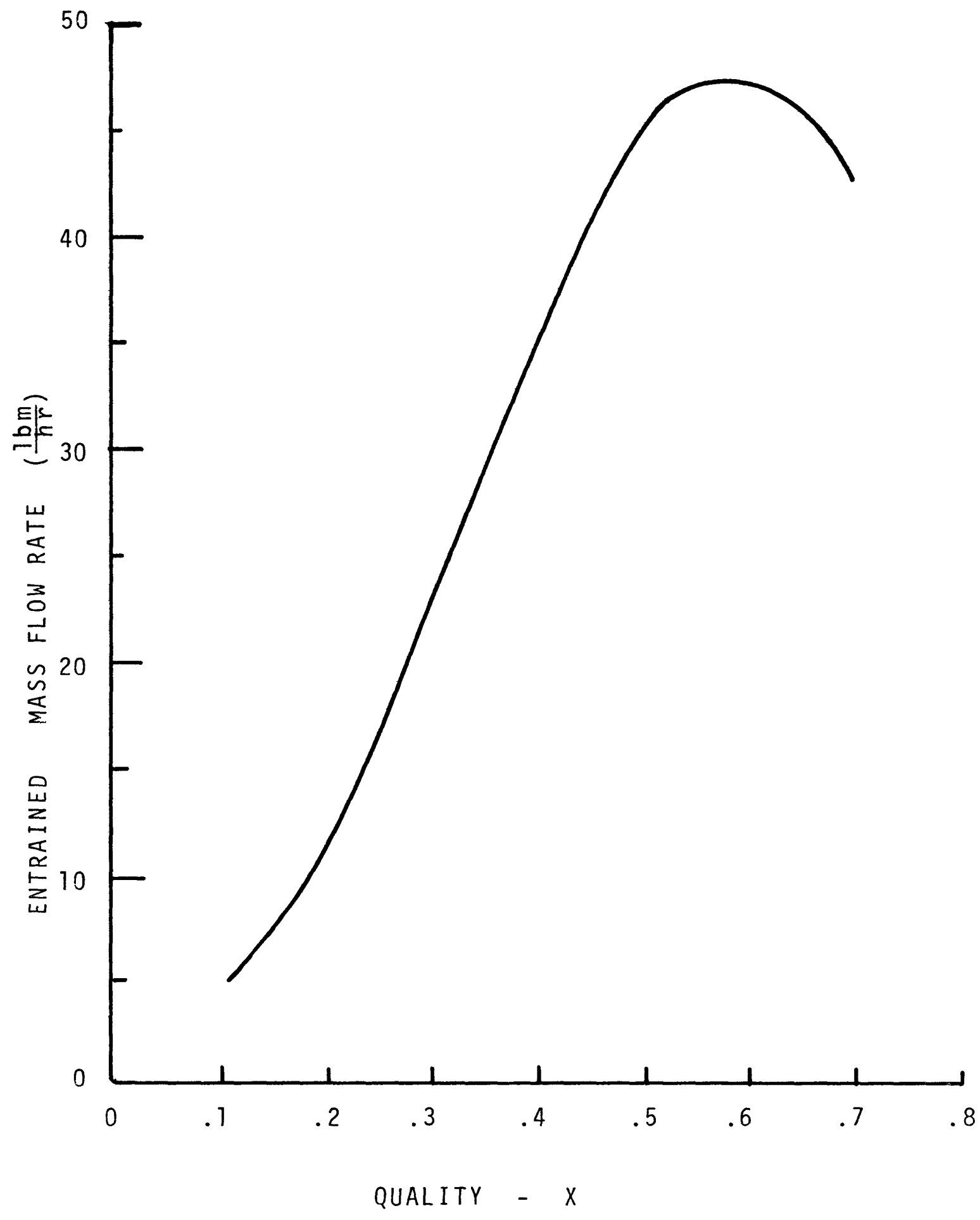

FIGURE A5-2 Hewitt's Entrainment Curve 


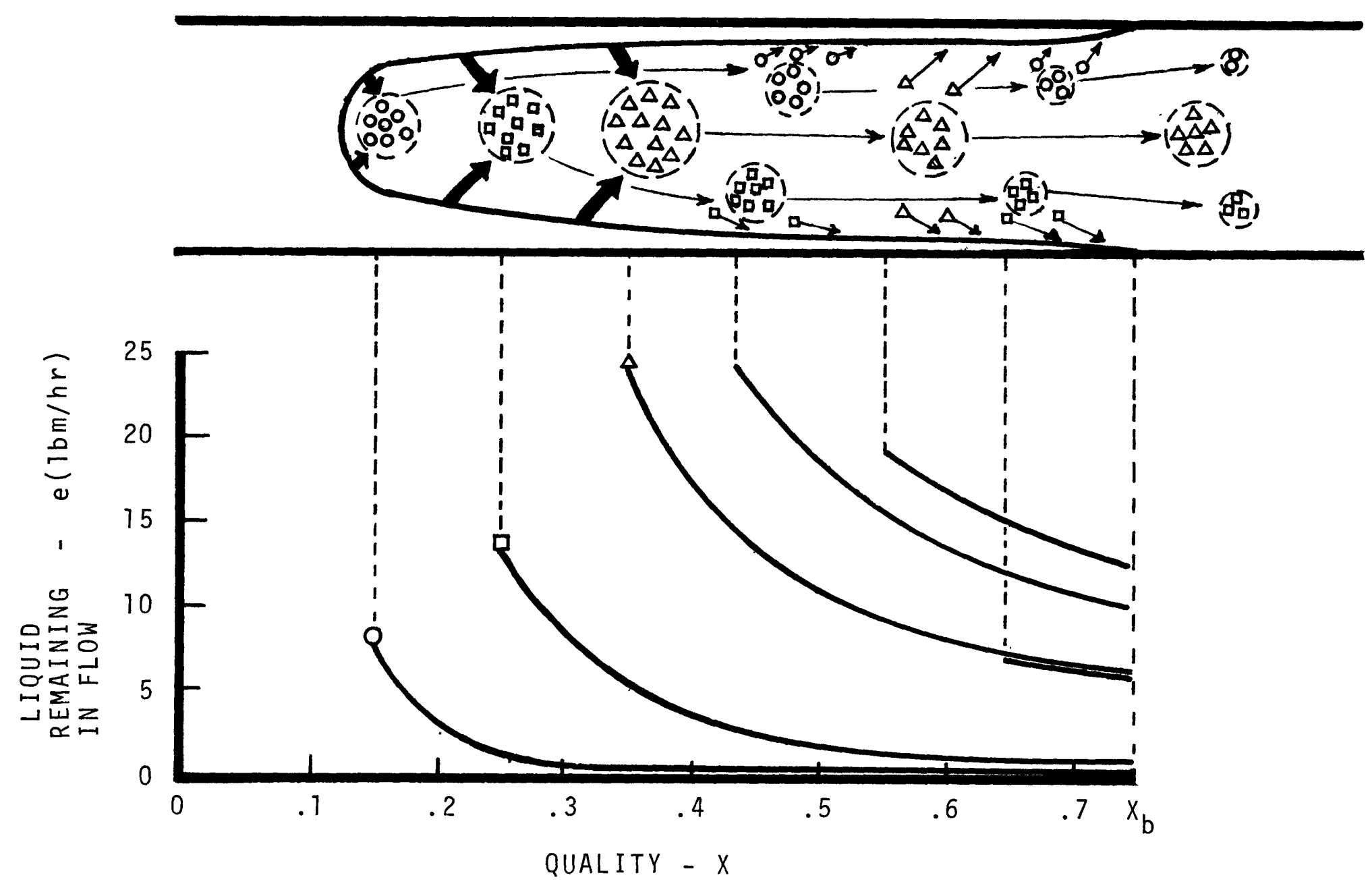

FJGURE A5-3 Single Sized Droplet Group History 
at any point in the tube thus equals the total entrained flow rate at that point.

At the burnout point, $x_{b}$, the remaining mass can be used to calculate the mass cumulative distribution, $E$. $E$ for the data of Figure $A 5-2$ is shown in Figure A5-4. As the figure shows, for this data, the assumption of a linear mass distribution function with respect to quality is reasonable. Drop sizes calculated using Figure A5-4 and that calculated using a linear $E$ agree to within approximate 1 y $12 \%$. 


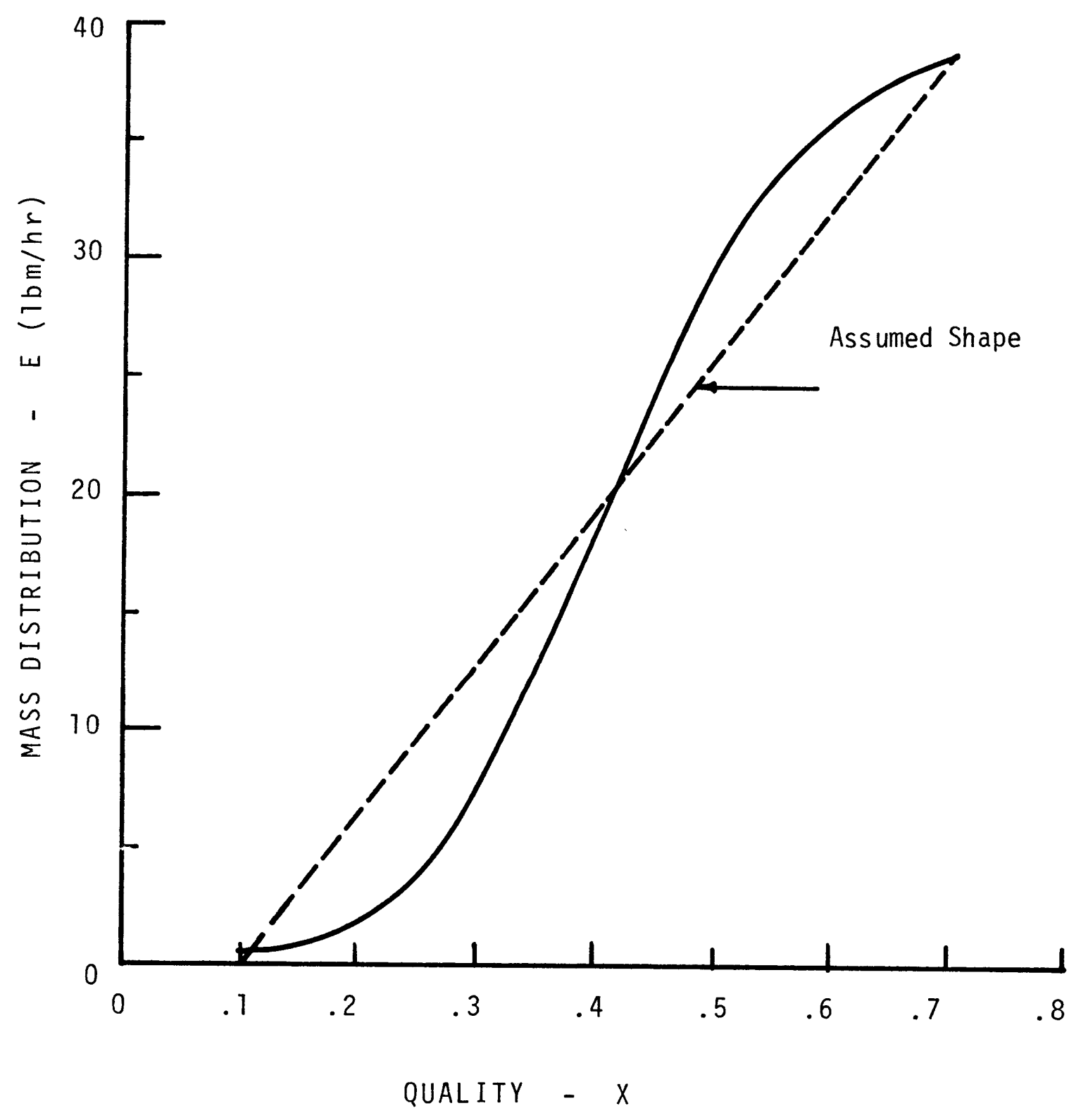

FIGURE A5-4 Cumulative Mass Distribution 


\section{REFERENCES}

1. Hynek S.J., "Forced Convection Dispersed Flow Film Boiling", Ph.D. Thesis, Massachusetts Institute of Technology, 1969 .

2. Bennet A.W., Hewitt G.F., Kearsey H.A., Keeys R.F.K., "Heat Transfer to Steam-Water Mixtures in Uniformly Heated Tubes in Which the Critical Heat Flux has been Exceeded "AERE-R 5373 .

3. Forslund R.P., "Thermal Non-Equilibrium in Dispersed Flow Film Boiling in a Vertical Tube", Ph.D. Thesis, Massachusetts Institute of Technology, December, 1966.

4. Iloeje 0.C., "A Study of Wall Rewet and Heat Transfer in Dispersed Vertical Flow", Ph.D. Thesis in Mechanical Engineering, Massachusetts Institute of Technology, February, 1975.

5. Groeneveld D.C., "The Thermal Behavior of a Heated Surface at and Beyond Dryout", AECL - 4309, 1972.

6. Cumo M., Farello G.E., Ferrari G., "The Influence of Curvature in Post Dryout Heat Transfer", Report of the XXVI National ATI Annual Meeting, L'Aquila, September, 1971.

7. Koizumi Y., Ueda T., Tanaka H., "Post Dryout Heat Transfer to R-113 Upward Flow in a Vertical Tube", Int. J. of Heat and Mass Transfer, Vol. 22, pp 669-678.

8. Miropolskiy A.L., "Heat Transfer in Film Boiling of a Steam-Water Mixture in Steam Generating Tubes," Teploenergetika, Vol. 10, 1963, pp. 49-53, (from [15]).

9. Polomik E.E., Levy S., Sawochka S.G., "Film Boiling of Steam-Water Mixtures in Annular Flow at 800,1100 , and 1400 Psi, ASME paper 62-WA-136.

10. Brevi R., "Post-Dryout Heat Transfer with Steam/Water Mixtures," Trans. Am. Nuc. Soc., Vol. 12, 1969, pp. $809-811$. 
12. Mattson R.J., Condie K.G., Bengston S.J. Obenchain C.F., "Regression Analysis of Post - CHF Flow Boiling Data," Int. Heat Tranf. Conf., Tokyo, 1974.

13. Chiang T., France D.M., Carlson R.D., "Post-CHF Heat Transfer and Transition Boiling Characteristics in an LMFBR Steam Generator Tube," ANL-CT-79-14.

14. Saha P., Shiralkar B.S., Dix G.E., "A Post-Dryout Heat Transfer Model Based on Actual Vapor Generation Rate in Dispersed Droplet Regime", ASMR paper 77-HT-80.

15. Groeneveld D.C., Gardiner S.R.M., "Post-CHF Heat Transfer Under Forced Convective Conditions," AEL-5883.

16. Dugall R.S., "Film Boiling on the Inside of Vertical Tubes with Upward Flow of the Fluid at Low Qualities," MIT Heat Transfer Laboratory Report No. 9079-26 (1963)

17. Laverty W.F., Rohsenow W.M., "Film Boiling of Saturated Liquid Nitrogen Flowing in a Vertical Tube," $\mathrm{J}$. of Heat Transfer, 89, pp. 90-98 (1967).

18. Plummer D.N., Iloeje O.C., Rohsenow W.M., Griffith P., Ganic E., "Post Critical Heat Transfer to Flowing Liquid in a Vertical Tube," MIT Heat Transfer Laboratory Report No. 72718-91, 1974.

19. Pederson C.0., "An Experimental Study of the Dynamic Behavior and Heat Transfer Characteristics of Water Droplets Impinging Upon a Heated Surface," Int. J. of Heat and Mass Transfer, V13, pp 369-381.

20. Ueda T., Enomoto T., Kanetsuki M., "Heat Transfer Characteristics and Dynamic Behavior of Saturated Droplets Impinging on a Heated Vertical Surface," Bulletin of the JSME, Vol. 22, No. 167, May, 1979, pp. 724-732.

21. Styricovich M.A., Baryshev, Yu V., Tsiklauri G.V., Grigorieva M.E., "The Mechanism of Heat and Mass Transfer Between a Water Drop and a Heated Surface," 
22. (McCarthy) Kendall G.E., Rohsenow W.M., "Heat Transfer to Impacting Drops and Post Critical Heat Flux Dispersed Flow, "MIT Heat Transfer Laboratory Report No. 85694-100, March 1978.

23. Liu Y.H., Ilori J.A., "Aerosol Deposition in Turbulent Pipe Flow," Environmental Science and Technology, Vol. 8, No. 4, April 1974, pp. 351-356.

24. Cumo M., Farrari G., Forello G.E., "A Photographic Study of Two-Phase Highly Dispersed Flows, "CNEN Report RT/ING(71)8, 1971 .

25. Lee R.S.L., Srinivasan J., "A Study of Droplet Hydrodynamics Important in LOCA Reflood," Paper presented at the NRC Two Phase Instrumentation Review Group Meeting, Troy, New York, March 13-14, 1978.

26. Ganic E.N., Rohsenow W.M., "Dispersed Flow Heat Transfer," Int. J. of Heat and Mass Transfer, Vol. 20, pp. $855-866,1977$.

27. Wicks and Duckler, "In Situ Measurements of Drop Size Distibution in Two-Phase Flow -- A new Method for Electrically Conducting Liquids," Paper presented at the International Heat Transfer Conference, Chicago, 1966 .

28. Mugele R.A., Evans H.D., "Droplet Size Distribution in Sprays," Industrial and Engineering Chemistry, Vol. 43, No. 6, pp. 1317-1324.

29. Tatterson D.F., Dallman J.C, Hanratty T.J., "Drop Sizes in Annular Gas-Liquid Flows," AIChE Journal, Vol. 23m No. 1, pp. 68-76.

30. Ishiki N., "Theoretical and Experimental Study on Atomization of Liquid Drops in a High Speed Gas Stream", Report No. 35, Transportation Technical Research Institute, Tokyo, Japan (from [18]).

31. Baum A., "A Study of Transition and Film Boiling from Vertical Surfaces," Ph.D. Thesis, Carnegie-Mellon University, April, 1977.

32. Hosler E.R., "Flow Patterns in High Pressure TwoPhase (Steam-Water) Flow with Heat Addition," Chemical Engineering Prog. Sym. Ser., 64, (1968), pp. 54-66, (from [31]). 
33. Ahmad S.Y., "Axial Distribution of Bulk Temperature and Void Fraction in a Heated Channel with Inlet Subcooling", Journal of Heat Transfer, Nov. 1970, pp. 595-609.

34. Wallis G.B., One Dimensional Two-Phase Flow, McGrawHi11, 1969 .

35. Hewitt G.F., Kearsey H.A., Lacey P.M.C., Pulling D.J., "Burnout and Film Flow in the Evaporation of Water in Tubes", AERE-R-4864, March, 1965.

36. Crowe C.T., "Conservation Equations for Vapor-Droplet Flows Including Boundary-Droplet Effects" UCRL-52184.

37. Ross L.L., Hoffman T.W., "Evaporation of Droplets in a High Temperature Environment", Paper presented at the Third International Heat Transfer Conference, Chicago, Aug. 1966, pp. 50-59.

38. Yuen M.C., Chen L.W., "Heat Transfer Measurements of Evaporating Liquid Droplets", Int. Journal of Heat and Mass Transfer, Vol. 21, pp. 537-542, 1978.

39. Ranz W.E., Marshall, "Evaporation from Drops I.II, Chemical Engineering Progress 48. 141-146, 173-180 (1952)

40. Hamelec A.E, Hoffman, T.W., Ross L.L., "Numerical Solution of the Navier-Stokes Equation for Flow Post Spheres. Part 1: Viscous Flow Around Spheres with and without Radial Mass Efflux", AIChE Journal, 13, 212.

41. Yuen M.C., Chen L.W., "On Drag of Evaporating Liquid Droplets", Combustion Science and Technology, Vo1. 14, pp. 147-154, 1976 .

42. Ingebo R.D., "Drag Coefficients for Droplets and Solid Spheres in Clouds Accelerating in Airstreams", NACA Technical Note - 3762, Sept., 1956.

43. Hayward G.L., Pei D.C., "Local Heat Transfer from a Single Sphere to a Turbulent Airstream", Int. Journal of Heat and Mass Transfer, Vol. 21, pp. 35-41, 1978. 
44. Rane A., Yao, S., "Heat Transfer of Evaporating Droplet Flow in Low Pressure Sys tems", ASME Paper, $79-W A / H T-10$.

45. Maisel D.S., Sherwood T.K., "Effect of Air Turbulence on Rate of Evaporation of Water", Chemical Engineering Progress, Vo1. 46, No. 4, pp. 172-175, Apri1, 1950.

46. Watchers L.H.J., Westerling N.A.J., "The Heat Transfer from a Hot Wall to Impinging Water Drops in the Spheroidal State," Chemical Engineering Science, 21 , pp 1047-1056 (1966).

47. Kays W.M., Convective Heat and Mass Transfer, McGraw$\mathrm{Hi} 11,1966$.

48. Sun K.H., Gonzalez J.M., Tien C.L., "Calculations of Combined Radiation and Convection Heat Transfer in Rod Bundles Under Emergency Cooling Conditions," ASME paper, 75-HT-64, 1975

49. Whalley P.B., Hutchinson P., Hewitt G.F., "The Calculation of Critical Heat Flux in Forced Convection Boiling," 5th-Int. Heat Transfer Conference, Japan, 1974. pp 290-294. 